\title{
Structurally Simple Benzylidene-Type Photolabile Diol Protecting Groups
}

Xiong Ding, Dattatray A. Devalankar, and Pengfei Wang*

Department of Chemistry, University of Alabama at Birmingham, Birmingham, Alabama 35294, United States

Table of Content

\begin{tabular}{|c|c|}
\hline & Page \\
\hline Table of content & S1 \\
\hline General procedure and materials & S2 \\
\hline Preparation of $\mathbf{5}$ and spectroscopic data & S2-3 \\
\hline Preparation of 1a and spectroscopic data & S3 \\
\hline Preparation of $\mathbf{1 b}$ and spectroscopic data & S3-4 \\
\hline Preparation of $\mathbf{1 c}$ and spectroscopic data & S4 \\
\hline Preparation of 1d and spectroscopic data & S5 \\
\hline Preparation of 1e and spectroscopic data & S5-6 \\
\hline Preparation of 1f and spectroscopic data & S6 \\
\hline Preparation of $1 \mathrm{~g}$ and spectroscopic data & S6-7 \\
\hline Preparation of $\mathbf{7}$ and $\mathbf{8}$, and spectroscopic data & S7 \\
\hline Preparation of $\mathbf{1 5}$ and, 10, and spectroscopic data & S7-8 \\
\hline Preparation of 11e and spectroscopic data & S8-9 \\
\hline Preparation of $11 \mathrm{~g}$ and $12 \mathrm{~g}$, and spectroscopic data & S9 \\
\hline General procedure for photolysis & S10 \\
\hline Quantum yield determination & S10 \\
\hline References & S10 \\
\hline${ }^{1} \mathrm{H}$ and ${ }^{13} \mathrm{C}$ NMR spectra of $\mathbf{1 a}$ & S11-12 \\
\hline${ }^{1} \mathrm{H}$ and ${ }^{13} \mathrm{C}$ NMR spectra of $\mathbf{1 b}$ & S13-14 \\
\hline${ }^{1} \mathrm{H}$ and ${ }^{13} \mathrm{C}$ NMR spectra of $\mathbf{1 c}$ & S15-16 \\
\hline${ }^{1} \mathrm{H}$ and ${ }^{13} \mathrm{C}$ NMR spectra of $\mathbf{1 c}$ & S17-18 \\
\hline${ }^{1} \mathrm{H}$ and ${ }^{13} \mathrm{C}$ NMR spectra of $\mathbf{1 d}$ & S19-20 \\
\hline${ }^{1} \mathrm{H}$ and ${ }^{13} \mathrm{C}$ NMR spectra of $\mathbf{1 e}$ & $\mathrm{S} 21-22$ \\
\hline${ }^{1} \mathrm{H}$ and ${ }^{13} \mathrm{C}$ NMR spectra of $\mathbf{1 e}$ & S23-24 \\
\hline${ }^{1} \mathrm{H}$ and ${ }^{13} \mathrm{C}$ NMR spectra of $\mathbf{1 f}$ & S25-26 \\
\hline${ }^{1} \mathrm{H}$ and ${ }^{13} \mathrm{C}$ NMR spectra of $\mathbf{1 f}$ & $\mathrm{S} 27-28$ \\
\hline${ }^{1} \mathrm{H}$ and ${ }^{13} \mathrm{C}$ NMR spectra of $\mathbf{1 g}$ & S29-30 \\
\hline${ }^{1} \mathrm{H}$ and ${ }^{13} \mathrm{C}$ NMR spectra of $\mathbf{5}$ & S31-32 \\
\hline${ }^{1} \mathrm{H}$ and ${ }^{13} \mathrm{C}$ NMR spectra of 7 & S33-34 \\
\hline${ }^{1} \mathrm{H}$ and ${ }^{13} \mathrm{C}$ NMR spectra of 8 & S35-36 \\
\hline${ }^{1} \mathrm{H}$ and ${ }^{13} \mathrm{C}$ NMR spectra of $\mathbf{1 0}$ & S37-38 \\
\hline${ }^{1} \mathrm{H}$ and ${ }^{13} \mathrm{C}$ NMR spectra of 11e & S39-40 \\
\hline${ }^{1} \mathrm{H}$ and ${ }^{13} \mathrm{C}$ NMR spectra of 11e' & S41-42 \\
\hline${ }^{1} \mathrm{H}$ and ${ }^{13} \mathrm{C}$ NMR spectra of $\mathbf{1 1 g}$ & S43-44 \\
\hline${ }^{1} \mathrm{H}$ and ${ }^{13} \mathrm{C}$ NMR spectra of $\mathbf{1 2 g}$ & S45-46 \\
\hline${ }^{1} \mathrm{H}$ and ${ }^{13} \mathrm{C}$ NMR spectra of $\mathbf{1 5}$ & S47-48 \\
\hline${ }^{1} \mathrm{H}$ and ${ }^{13} \mathrm{C}$ NMR spectra of $\mathbf{S 1}$ & S49-50 \\
\hline
\end{tabular}




\section{Experimental Section}

General. Organic solutions were concentrated by rotary evaporation at ca. 12 Torr. Flash column chromatography was performed employing 230-400 mesh silica gel. Thin-layer chromatography was performed using glass plates pre-coated to a depth of $0.25 \mathrm{~mm}$ with $230-400$ mesh silica gel impregnated with a fluorescent indicator $(254 \mathrm{~nm})$. Infrared (IR) data are presented as frequency of absorption $\left(\mathrm{cm}^{-1}\right)$. Proton and carbon-13 nuclear magnetic resonance $\left({ }^{1} \mathrm{H}\right.$ NMR or ${ }^{13} \mathrm{C}$ NMR) spectra were recorded on 300, 400 and $700 \mathrm{MHz}$ NMR spectrometers; Chemical shifts are expressed in parts per million ( $\delta$ scale) downfield from tetramethylsilane and are referenced to residual protium in the NMR solvent $\left(\mathrm{CHCl}_{3}: \delta\right.$ 7.26). Data are presented as follows: chemical shift, multiplicity $(\mathrm{s}=$ singlet, $\mathrm{d}=$ doublet, $\mathrm{t}=$ triplet, $\mathrm{q}=$ quartet, $\mathrm{m}=$ multiplet and $/$ or multiple resonances), coupling constant in Hertz $(\mathrm{Hz})$, integration.

Materials. Anhydrous solvents Tetrahydrofuran (THF), dimethylformamide (DMF), and dichloromethane (DCM) were used without distillation. Solvents for workup and column chromatography, such as petroleum ether (PE), hexanes (Hex), ethyl acetate (EA), methanol $(\mathrm{MeOH})$, benzene, toluene (Tol) and triethylamine (TEA), camphor sulfonic acid (CAS), and other chemicals were obtained from commercial vendors and used without further purification.

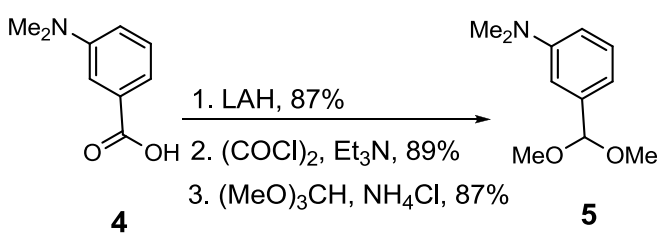

To a solution of 3-dimethylaminobenzoic acid $4(5 \mathrm{~g}, 30.3 \mathrm{mmol})$ in THF (100.0 mL), $\mathrm{LiAlH}_{4}$ $(1.7 \mathrm{~g}, 45.5 \mathrm{mmol})$ was added at room temperature under nitrogen atmosphere. The reaction mixture was stirred for $19 \mathrm{~h}$, quenched with statured solution of $\mathrm{NH}_{4} \mathrm{Cl}$, and filtered through sintered crucible. The filtrate was concentrated to provide the corresponding 3dimethylaminobenzyl alcohol $(3.9 \mathrm{~g}, 87 \%)$ as a brown liquid which was used for next step without further purification.

To a stirred solution of oxalyl chloride $(4.3 \mathrm{~mL}, 51.7 \mathrm{mmol})$ in dry DCM $(150.0 \mathrm{~mL})$, DMSO $(5.5 \mathrm{~mL}, 77.5 \mathrm{mmol})$ was added at $-78{ }^{\circ} \mathrm{C}$ under nitrogen atmosphere. The reaction mixture was stirred for $15 \mathrm{~min}$. followed by the addition of dimethylaminobenzyl alcohol $(3.9 \mathrm{~g}, 25.8 \mathrm{mmol})$. After stirring for $1 \mathrm{~h}$ at $-78{ }^{\circ} \mathrm{C}$, triethyl amine $(14.4 \mathrm{~mL}, 103.3 \mathrm{mmol})$ was added and reaction mixture was stirred at room temperature for additional $15 \mathrm{~min}$, after which it was quenched with $\mathrm{H}_{2} \mathrm{O}(100 \mathrm{~mL})$. The organic phase was separated and the aqueous phase was extracted with DCM $\left(2 \times 50 \mathrm{~mL}\right.$ ), the combined organic phase was dried over anhydrous $\mathrm{Na}_{2} \mathrm{SO}_{4}$ and concentrated under reduced pressure to give the corresponding crude benzaldehyde. The crude product was purified by column chromatography (PE/EA 19:1) to afford the product as a yellow liquid (3.4 $\mathrm{g}$, 89\%): $\mathrm{Rf}=0.5\left(\mathrm{PE} / \mathrm{EA} \mathrm{19:1);}{ }^{1} \mathrm{H} \mathrm{NMR}\left(400 \mathrm{MHz}, \mathrm{CDCl}_{3}\right) \delta 9.96(\mathrm{~s}, 1 \mathrm{H}), 7.39\right.$ (t, $J=7.5 \mathrm{~Hz}$, $1 \mathrm{H}), 7.19(\mathrm{~m}, 2 \mathrm{H}), 6.69-6.99(\mathrm{~m}, 2 \mathrm{H}), 3.02(\mathrm{~s}, 6 \mathrm{H}) ;{ }^{13} \mathrm{C} \mathrm{NMR}\left(75 \mathrm{MHz}, \mathrm{CDCl}_{3}\right) \delta$ 193.2, 150.7, 
137.2, 129.6, 118.9, 118.3, 111.5, 40.44; IR (neat) 2808, 1696, 1600, 1498, 1356, 1205; HRMS (ESI) m/e calcd for $\mathrm{C}_{9} \mathrm{H}_{12} \mathrm{NO}(\mathrm{M}+\mathrm{H}) 150.0919$, found 150.0922 .

To a solution of 3-dimethylaminobenzaldehyde $(1.5 \mathrm{~g}, 10.1 \mathrm{mmol})$ and trimethylorthoformate $(5.9 \mathrm{~mL}, 50.3 \mathrm{mmol})$ in methanol $(25 \mathrm{~mL})$ was added $\mathrm{NH}_{4} \mathrm{Cl}(16 \mathrm{mg}, 0.3 \mathrm{mmol})$ at room temperature. Reaction mixture was stirred at room temperature for $72 \mathrm{~h}$ and the reaction solution was concentrated. The crude mixture was purified by column chromatography (benzene/EA 95:5) to afford the acetal $5(1.8 \mathrm{~m}, 87 \%)$ as a yellow liquid: $\mathrm{Rf}=0.5$ (benzene/EA 95:5); ${ }^{1} \mathrm{H}$ NMR (400 MHz, $\left.\mathrm{CDCl}_{3}\right) \delta 7.25(\mathrm{t}, J=7.8 \mathrm{~Hz}, 1 \mathrm{H}), 6.83(\mathrm{~m}, 2 \mathrm{H}), 6.69$ (dd, $J=8.0,2.5 \mathrm{~Hz}, 1 \mathrm{H}$ ), $5.33(\mathrm{~s}, 1 \mathrm{H}), 3.34(\mathrm{~s}, 6 \mathrm{H}), 2.96(\mathrm{~s}, 6 \mathrm{H}) ;{ }^{13} \mathrm{C} \mathrm{NMR}\left(75 \mathrm{MHz}, \mathrm{CDCl}_{3}\right)$ 8150.5, 138.7, 128.8, 115.0, 112.6, 110.6, 103.7, 52.7, 40.6; IR (neat) 2935, 2887, 2827, 1604, 1582, 1497, 1435, 1350; HRMS (ESI) m/e calcd for $\mathrm{C}_{11} \mathrm{H}_{18} \mathrm{NO}_{2}(\mathrm{M}+\mathrm{H})$ 196.1338, found 196.1342 .
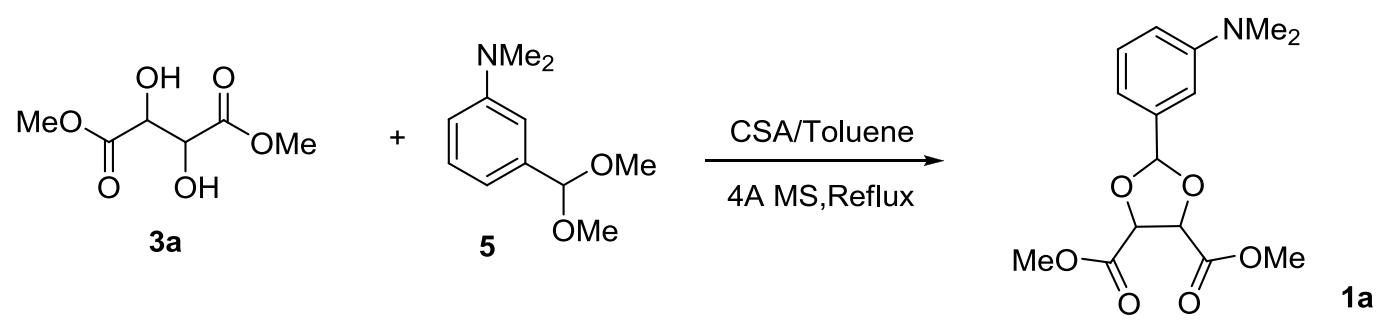

To a solution of the diol 3a (134 mg, $0.75 \mathrm{mmol})$ and the PPG reagent $5(293 \mathrm{mg}, 1.50 \mathrm{mmol})$ in toluene $(2 \mathrm{~mL})$ was added a catalytic amount of CSA $(35 \mathrm{mg}, 0.15 \mathrm{mmol})$ and $4 \mathrm{~A}$ molecular sieve $(25 \mathrm{mg})$ at room temperature under $\mathrm{N}_{2}$. The reaction mixture was refluxed for $24 \mathrm{~h}$. After completion of reaction, the reaction solution was neutralized with TEA at room temperature and concentrated under reduced pressure. The crude reaction mixture was purified by column chromatography (PE/EA 10:1) to provide the desired product 1a (165 mg, 71\%) as a colorless liquid: $\mathrm{Rf}=0.25\left(\mathrm{PE} / \mathrm{EA} \mathrm{10:1);}{ }^{1} \mathrm{H} \mathrm{NMR}\left(400 \mathrm{MHz}, \mathrm{CDCl}_{3}\right) \delta 7.26(\mathrm{t}, J=7.8 \mathrm{~Hz}, 1 \mathrm{H}), 6.96(\mathrm{~s}\right.$, $1 \mathrm{H}), 6.90(\mathrm{~d}, J=7.5 \mathrm{~Hz}, 1 \mathrm{H}), 6.77(\mathrm{dd}, J=8.2,2.0 \mathrm{~Hz}, 1 \mathrm{H}), 6.11(\mathrm{~s}, 1 \mathrm{H}), 4.98(\mathrm{~d}, J=4.0 \mathrm{~Hz}$, $1 \mathrm{H}), 4.86(\mathrm{~d}, \mathrm{~J}=3.7 \mathrm{~Hz}, 1 \mathrm{H}), 3.87(\mathrm{~s}, 3 \mathrm{H}), 3.82(\mathrm{~s}, 3 \mathrm{H}), 2.96(\mathrm{~s}, 6 \mathrm{H}) ;{ }^{13} \mathrm{C}$ NMR $(176 \mathrm{MHz}$, $\left.\mathrm{CDCl}_{3}\right) \delta 170.2,169.6,150.7,136.0,129.2,115.3,114.2,111.0,107.3,52.9,40.6$; IR (neat) 2954, 2905, 2803, 1754, 1677, 1609, 1585, 1503, 1438; HRMS (ESI) m/e calcd for $\mathrm{C}_{15} \mathrm{H}_{20} \mathrm{NO}_{6}$ $(\mathrm{M}+\mathrm{H}) 310.1291$, found 310.1285 .

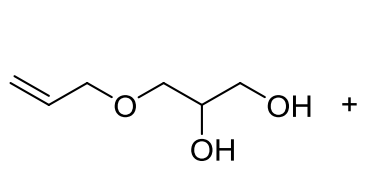

3b
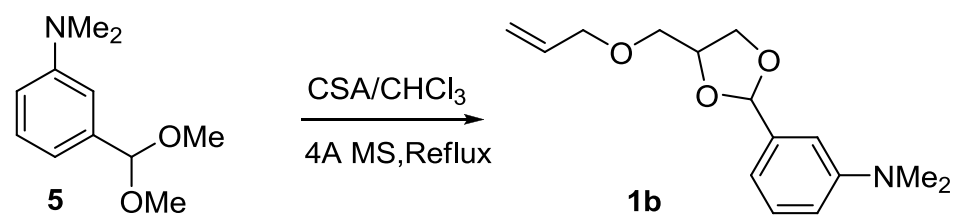

To a solution of the diol $\mathbf{3 b}(70 \mathrm{mg}, 0.530 \mathrm{mmol})$ and the PPG reagent $5(155 \mathrm{mg}, 0.795 \mathrm{mmol})$ in $\mathrm{CHCl}_{3}(3 \mathrm{~mL})$ was added a catalytic amount of CSA $(25 \mathrm{mg}, 0.106 \mathrm{mmol})$ and $4 \mathrm{~A}$ molecular 
sieve $(25 \mathrm{mg})$ at room temperature under $\mathrm{N}_{2}$. The reaction mixture was refluxed for $4 \mathrm{~h}$. After completion of the reaction, the reaction mixture was neutralized with TEA and then concentrated under reduced pressure. The crude product was purified by column chromatography (PE/EA 20:1) to provide the desired product $\mathbf{1 b}(121 \mathrm{mg}, 87 \%)$ as a colorless liquid: $\mathrm{Rf}=0.2$ (PE/EA 20:1); ${ }^{1} \mathrm{H}$ NMR $\left(400 \mathrm{MHz}, \mathrm{CDCl}_{3}\right) \delta 7.22(\mathrm{~m}, 1 \mathrm{H}), 6.84(\mathrm{~m}, 2 \mathrm{H}), 6.72(\mathrm{~m}, 1 \mathrm{H}), 5.78-5.94(\mathrm{~m}$, $2 \mathrm{H}), 5.30-5.33(\mathrm{~m}, 1 \mathrm{H}), 5.16-5.22(\mathrm{~m}, 1 \mathrm{H}), 4.38-4.46(\mathrm{~m}, 1 \mathrm{H}), 3.98-4.26(\mathrm{~m}, 4 \mathrm{H}), 3.52-3.67(\mathrm{~m}$, 2H), $2.96(\mathrm{~s}, 6 \mathrm{H}) ;{ }^{13} \mathrm{C} \mathrm{NMR}\left(75 \mathrm{MHz}, \mathrm{CDCl}_{3}\right) \delta 150.6,138.6,138.0,134.4,129.0,117.3,114.7$, 114.5, 113.5, 113.3, 110.5, 110.2, 104.7, 104.1, 75.2, 74.8, 72.4, 70.9, 70.4, 67.9, 67.6, 40.6; IR (neat) 2872, 2804, 1680, 1607, 1584, 1500, 1440; HRMS (ESI) m/e calcd for $\mathrm{C}_{15} \mathrm{H}_{22} \mathrm{NO}_{3}(\mathrm{M}+\mathrm{H})$ 264.1600, found 264.1603.
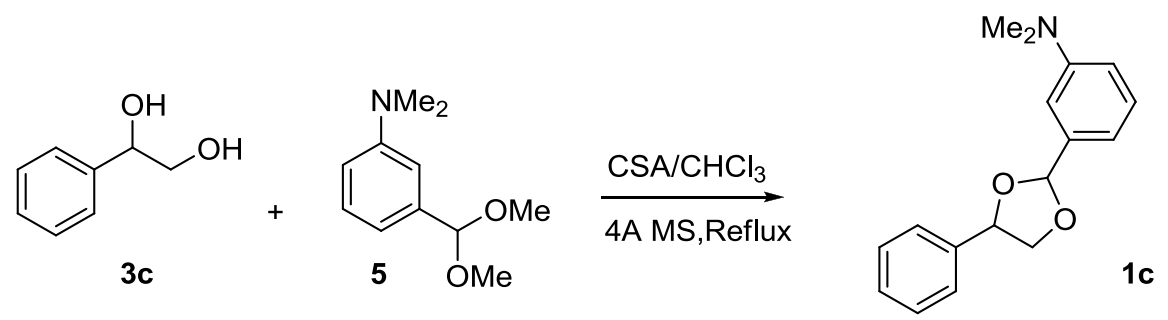

To a solution of the diol 3c $(69 \mathrm{mg}, 0.50 \mathrm{mmol})$ and the PPG $5(146 \mathrm{mg}, 0.75 \mathrm{mmol})$ in $\mathrm{CHCl}_{3}(3$ $\mathrm{mL})$ was added catalytic amount of CSA (23 mg, $0.10 \mathrm{mmol})$ and 4A MS (25 mg) at room temperature under $\mathrm{N}_{2}$. The reaction mixture was refluxed for $5 \mathrm{~h}$. After completion of reaction, the reaction solution was neutralized with TEA at room temperature and then concentrated under reduced pressure. The crude product was purified by column chromatography (PE/EA 100:1 to 20:1) to provide product $1 \mathbf{c}(29 \mathrm{mg})$ and its diastereomer 1 $\mathbf{1}$ ' $(98 \mathrm{mg})$ as a colorless liquid with a combined $94 \%$ yield.

For 1c, $\mathrm{Rf}=0.5(\mathrm{PE} / \mathrm{EA} 50: 1) ;{ }^{1} \mathrm{H}$ NMR $\left(400 \mathrm{MHz}, \mathrm{CDCl}_{3}\right) \delta$ 7.43-7.44 $(\mathrm{m}, 2 \mathrm{H}), 7.25-7.38(\mathrm{~m}$, $5 \mathrm{H}), 6.94(\mathrm{~m}, 2 \mathrm{H}), 6.77(\mathrm{~m}, 1 \mathrm{H}), 5.98(\mathrm{~s}, 1 \mathrm{H}), 5.20(\mathrm{t}, J=7.0 \mathrm{~Hz}, 1 \mathrm{H}), 4.37(\mathrm{t}, J=7.0 \mathrm{~Hz}, 1 \mathrm{H})$, $3.94(\mathrm{t}, J=6.5 \mathrm{~Hz}, 1 \mathrm{H}), 2.96(\mathrm{~s}, 6 \mathrm{H}) ;{ }^{13} \mathrm{C} \mathrm{NMR}\left(75 \mathrm{MHz}, \mathrm{CDCl}_{3}\right) \delta 150.7,139.6,139.0,129.1$, 128.6, 128.0, 126.1, 114.5, 113.4, 110.2, 104.9, 77.8, 72.7, 40.6; IR (neat) 3031, 2871, 2804, 1738, 1679, 1606, 1584, 1497; HRMS (ESI) m/e calcd for $\mathrm{C}_{17} \mathrm{H}_{20} \mathrm{NO}_{2}(\mathrm{M}+\mathrm{H}) 270.1494$, found 270.1495 .

For 1c', Rf $=0.45\left(\right.$ PE/EA 50:1); ${ }^{1} \mathrm{H}$ NMR $\left(400 \mathrm{MHz}, \mathrm{CDCl}_{3}\right) \delta$ 7.26-7.43 (m. 6H), $6.90(\mathrm{~m}$, 2H), $6.75(\mathrm{~d}, J=7.3 \mathrm{~Hz}, 1 \mathrm{H}), 6.18(\mathrm{~s}, 1 \mathrm{H}), 5.22(\mathrm{t}, J=6.8 \mathrm{~Hz}, 1 \mathrm{H}), 4.52(\mathrm{t}, J=7.0 \mathrm{~Hz}, 1 \mathrm{H}), 3.87$ $(\mathrm{t}, J=8.3 \mathrm{~Hz}, 1 \mathrm{H}), 2.97(\mathrm{~s}, 6 \mathrm{H}) ;{ }^{13} \mathrm{C}$ NMR $\left(75 \mathrm{MHz}, \mathrm{CDCl}_{3}\right) \delta 150.6,139.3,138.2,129.1,128.5$, 128.1, 126.4, 114.8, 113.5, 110.6, 105.0, 78.7, 72.1, 40.6; IR (neat) 3031, 2879, 2805, 1679, 1607, 1583, 1500, 1453; HRMS (ESI) m/e calcd for $\mathrm{C}_{17} \mathrm{H}_{20} \mathrm{NO}_{2}(\mathrm{M}+\mathrm{H})$ 270.1494, found 270.1497 . 


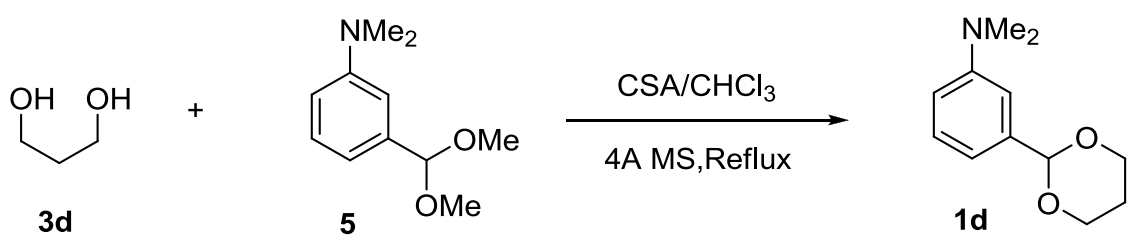

To a solution of the diol 3d $(45 \mathrm{mg}, 0.591 \mathrm{mmol})$ and the PPG reagent $\mathbf{5}(173 \mathrm{mg}, 0.887 \mathrm{mmol})$ in $\mathrm{CHCl}_{3}(3 \mathrm{~mL})$ was added a catalytic amount of CSA $(27 \mathrm{mg}, 0.118 \mathrm{mmol})$ and $4 \mathrm{~A}$ molecular sieve $\left(25 \mathrm{mg}\right.$ ) under $\mathrm{N}_{2}$ at room temperature. The reaction mixture was refluxed for $2 \mathrm{~h}$, and was then neutralized with TEA at room temperature. The solution was concentrated under reduced pressure and the crude reaction mixture was purified by column chromatography (PE/EA 10:1) to provide 1d (109 $\mathrm{mg}, 89 \%)$ as a colorless liquid: $\mathrm{Rf}=0.25$ (PE/EA 4:1); ${ }^{1} \mathrm{H}$ NMR (400 MHz, $\left.\mathrm{CDCl}_{3}\right) \delta 7.22(\mathrm{t}, J=7.8 \mathrm{~Hz}, 1 \mathrm{H}), 6.83(\mathrm{~m}, 2 \mathrm{H}), 6.72(\mathrm{dd}, J=8.0,2.5 \mathrm{~Hz}, 1 \mathrm{H}), 5.46(\mathrm{~s}, 1 \mathrm{H})$, $4.26(\mathrm{dd}, J=10.8,5.0 \mathrm{~Hz}, 2 \mathrm{H}), 3.98$ (dt, $J=11.8,2.0 \mathrm{~Hz}, 2 \mathrm{H}), 2.94$ (s, 6H), 2.17-2.29 (m, 1H), $1.45(\mathrm{~d}, J=13.3 \mathrm{~Hz}, 1 \mathrm{H}) ;{ }^{13} \mathrm{C} \mathrm{NMR}\left(75 \mathrm{MHz}, \mathrm{CDCl}_{3}\right) \delta 150.6,139.4,128.9,114.3,113.2$, 109.9, 102.1, 67.3, 40.6, 25.7; IR (neat) 2985, 2922, 2850, 2802, 1680, 1608, 1584, 1499, 1438; HRMS (ESI) m/e calcd for $\mathrm{C}_{12} \mathrm{H}_{18} \mathrm{NO}_{2}(\mathrm{M}+\mathrm{H})$ 208.1338, found 208.1342.

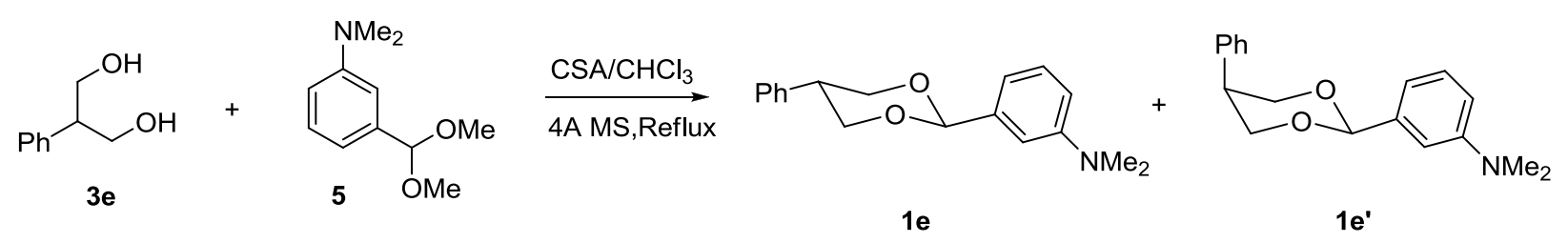

To a solution of the diol 3e $(99 \mathrm{mg}, 0.650 \mathrm{mmol})$ and the PPG reagent 5 (190 $\mathrm{mg}, 0.975 \mathrm{mmol})$ in $\mathrm{CH}_{3} \mathrm{Cl}(3 \mathrm{~mL})$ was added a catalytic amount of CSA $(30 \mathrm{mg}, 0.130 \mathrm{mmol})$ and $4 \mathrm{~A}$ MS $(25$ $\mathrm{mg}$ ) at room temperature under $\mathrm{N}_{2}$. The reaction mixture was refluxed for $2 \mathrm{~h}$. After completion of reaction, the reaction mixture was neutralized with TEA at room temperature and then was concentrated under reduced pressure. The crude product was purified by column chromatography (PE/EA 50:1 to 20:1) to provide 1e (115 mg) and its diastereomer 1e' (42 $\mathrm{mg}$ ) as colorless liquid in a combined $85 \%$ yield.

For 1e, $\mathrm{Rf}=0.4(\mathrm{PE} / \mathrm{EA} 20: 1) ;{ }^{1} \mathrm{H} \mathrm{NMR}\left(400 \mathrm{MHz}, \mathrm{CDCl}_{3}\right) \delta 7.34(\mathrm{~m}, 2 \mathrm{H}), 7.23-7.30(\mathrm{~m}, 5 \mathrm{H})$, $6.93(\mathrm{~s}, 1 \mathrm{H}), 6.88(\mathrm{~d}, J=8.0 \mathrm{~Hz}, 1 \mathrm{H}), 6.74(\mathrm{dd}, J=8.0,4.0 \mathrm{~Hz}, 1 \mathrm{H}), 5.55(\mathrm{~s}, 1 \mathrm{H}), 4.37(\mathrm{dd}, J=$ 12.0, $4.0 \mathrm{~Hz}, 2 \mathrm{H}), 4.04(\mathrm{t}, J=12.0 \mathrm{~Hz}, 2 \mathrm{H}) 3.43-3.35(\mathrm{~m}, 1 \mathrm{H}), 2.97(\mathrm{~s}, 6 \mathrm{H}) ;{ }^{13} \mathrm{C} \mathrm{NMR}(75 \mathrm{MHz}$, $\left.\mathrm{CDCl}_{3}\right) \delta 150.8,139.0,137.8,129.1,128.8,127.7,127.4,114.5,113.4,110.1,102.1,72.4,41.2$, 40.7; IR (neat) 3029, 2959, 2917, 2850, 2802, 1679, 1605, 1583, 1495; HRMS (ESI) m/e calcd for $\mathrm{C}_{18} \mathrm{H}_{22} \mathrm{NO}_{2}(\mathrm{M}+\mathrm{H})$ 284.1651, found 284.1652.

For 1e', Rf = $0.35(\mathrm{PE} / \mathrm{EA} 20: 1) ;{ }^{1} \mathrm{H}$ NMR $\left(400 \mathrm{MHz}, \mathrm{CDCl}_{3}\right) \delta 7.62(\mathrm{~d}, \mathrm{~J}=7.7,2 \mathrm{H}), 7.33(\mathrm{t}, \mathrm{J}$ = 7.7, $2 \mathrm{H}), 7.26-7.24(\mathrm{~m}, 2 \mathrm{H}), 6.91(\mathrm{~s}, 1 \mathrm{H}), 6.89(\mathrm{~d}, J=7.7 \mathrm{~Hz}, 1 \mathrm{H}), 6.73(\mathrm{dd}, J=8.4,2.1 \mathrm{~Hz}$, $1 \mathrm{H}), 5.63(\mathrm{~s}, 1 \mathrm{H}), 4.39-4.34(\mathrm{~m}, 4 \mathrm{H}), 2.95(\mathrm{~s}, 6 \mathrm{H}), 2.76(\mathrm{~s}, 1 \mathrm{H}) ;{ }^{13} \mathrm{C} \mathrm{NMR}\left(176 \mathrm{MHz}, \mathrm{CDCl}_{3}\right)$ 
$\delta 150.8,142.9,139.3,129.1,128.6,128.3,126.5,114.5,113.3,110.4,102.1,71.1,40.7,39.4$; IR (neat); HRMS (ESI) m/e calcd for $\mathrm{C}_{18} \mathrm{H}_{22} \mathrm{NO}_{2}(\mathrm{M}+\mathrm{H}) 284.1651$, found 284.1648.

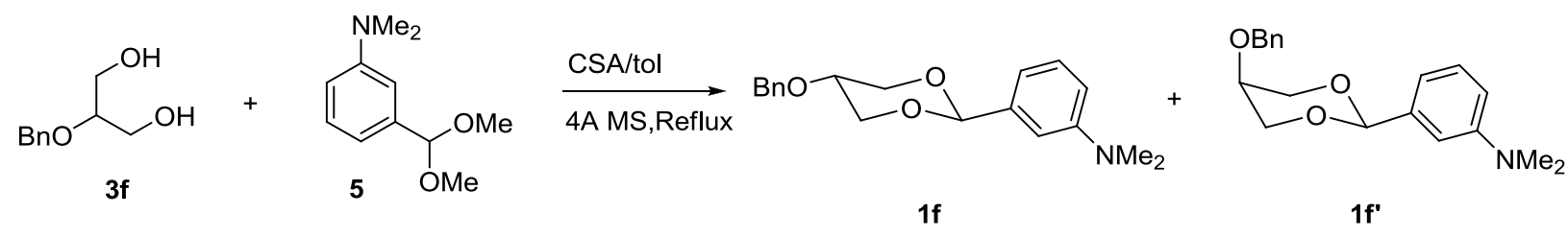

To a solution of the diol $\mathbf{3 f}(120 \mathrm{mg}, 0.659 \mathrm{mmol})$ and the PPG reagent 5 (193 $\mathrm{mg}, 0.988 \mathrm{mmol})$ in toluene $(2 \mathrm{~mL})$ was added a catalytic amount of CSA $(31 \mathrm{mg}, 0.132 \mathrm{mmol})$ and 4A MS (25 $\mathrm{mg}$ ) at room temperature under $\mathrm{N}_{2}$. The reaction mixture was refluxed for $3.5 \mathrm{~h}$. After completion of reaction, the reaction mixture was neutralized with TEA at room temperature and then was concentrated under reduced pressure. The crude product was purified by column chromatography (PE/EA 20:1 to 5:1) to provide $1 \mathrm{~g}(138 \mathrm{mg})$ and its isomer 1g' $(50 \mathrm{mg})$ as a colorless liquid in a combined $91 \%$ yield.

For 1f, $\mathrm{Rf}=0.2(\mathrm{PE} / \mathrm{EA} 20: 1) ;{ }^{1} \mathrm{H}$ NMR $\left(400 \mathrm{MHz}, \mathrm{CDCl}_{3}\right) \delta$ 7.31-7.39 (m. 5H), 7.19-7.25 (t, $J$ $=7.8 \mathrm{~Hz}, 1 \mathrm{H}), 6.81(\mathrm{~m}, 2 \mathrm{H}), 6.70(\mathrm{dd}, J=8.3,2.7 \mathrm{~Hz}, 1 \mathrm{H}), 5.35(\mathrm{~s}, 1 \mathrm{H}), 4.60(\mathrm{~s}, 2 \mathrm{H}), 4.35(\mathrm{dd}, \mathrm{J}$ $=10.6,4.8 \mathrm{~Hz}, 2 \mathrm{H}), 3.79(\mathrm{~m}, 1 \mathrm{H}), 3.62-3.67(\mathrm{~m}, 2 \mathrm{H}), 2.94(\mathrm{~s}, 6 \mathrm{H}) ;{ }^{13} \mathrm{C} \mathrm{NMR}\left(75 \mathrm{MHz}, \mathrm{CDCl}_{3}\right) \delta$ 150.6, 136.3, 137.9, 128.9, 128.5, 128.0, 127.7, 114.3, 113.3, 109.9, 101.8, 71.7, 70.1, 67.9, 40.6; IR (neat) 2858, 1678, 1608, 1100; HRMS (ESI) m/e calcd for $\mathrm{C}_{19} \mathrm{H}_{24} \mathrm{NO}_{3}(\mathrm{M}+\mathrm{H})$ 314.1756 , found 314.1755 .

For 1f', Rf = $0.5(\mathrm{PE} / \mathrm{EA} 5: 1) ;{ }^{1} \mathrm{H}$ NMR $\left(400 \mathrm{MHz}, \mathrm{CDCl}_{3}\right) \delta$ 7.20-7.42 (m, 6H), $6.91(\mathrm{~s}, 1 \mathrm{H})$, $6.86(\mathrm{~d}, J=7.3 \mathrm{~Hz}, 1 \mathrm{H}), 6.71(\mathrm{dd}, J=8.0,2.2 \mathrm{~Hz}, 1 \mathrm{H}), 5.52(\mathrm{~s}, 1 \mathrm{~h}), 4.71(\mathrm{~s}, 2 \mathrm{H}), 4.35$ (d, $J=$ $12.3 \mathrm{~Hz}, 2 \mathrm{H}), 4.02(\mathrm{~d}, J=12.6 \mathrm{~Hz}, 2 \mathrm{H}), 3.34(\mathrm{~s}, 1 \mathrm{H}), 2.95(\mathrm{~s}, 6 \mathrm{H}) ;{ }^{13} \mathrm{C}$ NMR $\left(75 \mathrm{MHz}, \mathrm{CDCl}_{3}\right)$ 8150.6, 138.8, 138.2, 128.8, 128.4, 127.7, 127.6, 114.6, 113.3, 110.2, 101.9, 70.2, 69.3, 68.9, 40.7; IR (neat) 2969; 2852, 1607, 14981343; HRMS (ESI) m/e calcd for $\mathrm{C}_{19} \mathrm{H}_{24} \mathrm{NO}_{3}(\mathrm{M}+\mathrm{H})$ 314.1756, found 314.1757 .

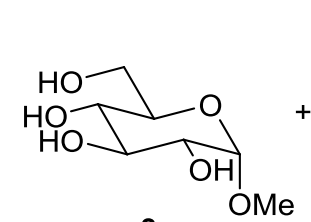

$3 g$

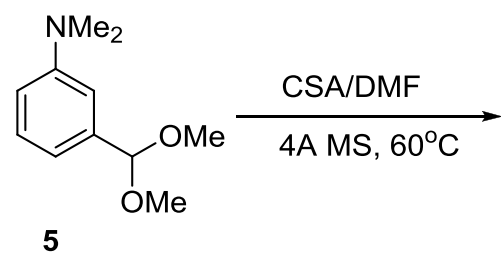

5

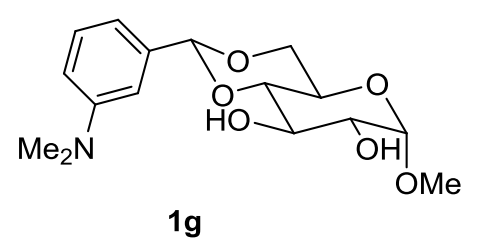

$1 \mathrm{~g}$

To a solution of the methyl glucoside $\mathbf{3 g}(97 \mathrm{mg}, 0.5 \mathrm{mmol})$ and the PPG reagent 5 (146 mg, $0.75 \mathrm{mmol})$ in DMF (2 mL) was added a catalytic amount of CSA $(23 \mathrm{mg}, 0.1 \mathrm{mmol})$ and $4 \mathrm{~A}$ molecular sieve $(25 \mathrm{mg})$ at room temperature under $\mathrm{N}_{2}$. The reaction mixture was stirred at $60{ }^{\circ} \mathrm{C}$ for $2 \mathrm{~h}$. After completion of reaction, the reaction mixture was neutralized with TEA and purified 
with column chromatography (DCM/MeOH 20:1) to produce $\mathbf{1 g}$ as a colorless liquid which solidifies at $0{ }^{\circ} \mathrm{C}(138 \mathrm{mg}, 85 \%) . \mathrm{Rf}=0.4(\mathrm{DCM} / \mathrm{MeOH}=20: 1) ;{ }^{1} \mathrm{H} \mathrm{NMR}\left(400 \mathrm{MHz}, \mathrm{CDCl}_{3}\right) \delta$ $7.21(\mathrm{t}, J=8.0 \mathrm{~Hz}, 1 \mathrm{H}), 6.82(\mathrm{~m}, 2 \mathrm{H}), 6.72(\mathrm{dd}, \mathrm{J}=8.0,2.5 \mathrm{~Hz}, 1 \mathrm{H}), 5.47(\mathrm{~s}, 1 \mathrm{H}), 4.78(\mathrm{~d}, J=$ $3.7 \mathrm{~Hz}, 1 \mathrm{H}), 4.29$ (dd, $J=10.1,4.8 \mathrm{~Hz}, 1 \mathrm{H}), 3.94(\mathrm{t}, J=9.3 \mathrm{~Hz}, 1 \mathrm{H}), 3.80(\mathrm{~m}, 1 \mathrm{H}), 3.72(\mathrm{~m}, 1 \mathrm{H})$, $3.63(\mathrm{~m}, 1 \mathrm{H}), 3.46(\mathrm{~s}, 3 \mathrm{H}), 2.95(\mathrm{~s}, 6 \mathrm{H}) ;{ }^{13} \mathrm{C} \mathrm{NMR}\left(75 \mathrm{MHz}, \mathrm{CDCl}_{3}\right) \delta 150.6,137.7,129.0$, $114.5,113.6,110.2,102.6,99.7,81.0,72.7,71.7,68.9,62.3,55.5,40.6$; IR (neat) 3433, 2912, 1608, 1501, 1073; HRMS (ESI) m/e calcd for $\mathrm{C}_{16} \mathrm{H}_{24} \mathrm{NO}_{6}(\mathrm{M}+\mathrm{H}) 326.1604$, found 326.1607.

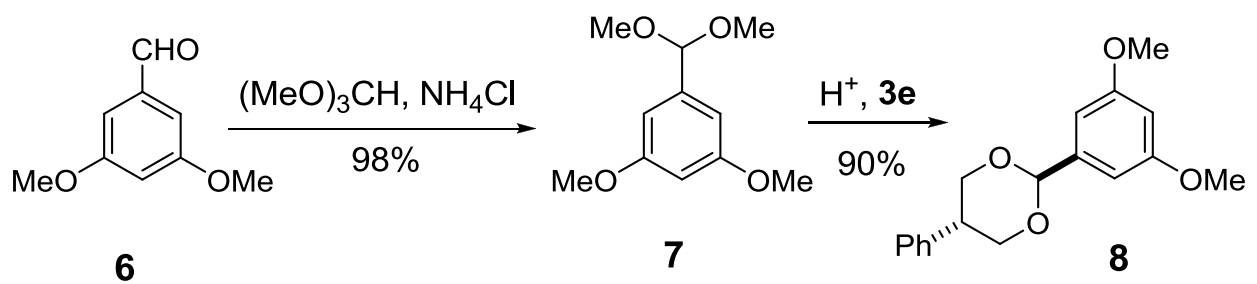

To a solution of the benzaldehyde $6(2.0 \mathrm{~g}, 12.0 \mathrm{mmol})$ and and trimethylorthoformate $(7.0 \mathrm{~mL}$, $60.2 \mathrm{mmol})$ in methanol $(20 \mathrm{~mL})$ was added $\mathrm{NH}_{4} \mathrm{Cl}(64.0 \mathrm{mg}, 1.2 \mathrm{mmol})$. The reaction mixture was stirred for $24 \mathrm{~h}$. After completion, the reaction mixture was concentrated under reduced pressure. The residue was purified by column chromatography (PE/EA 19:1) to provide the desired acetal $7(2.4 \mathrm{~g}, 98 \%)$ as a colorless oil. $\mathrm{Rf}=0.5(\mathrm{PE} / \mathrm{EA}=19: 1) ;{ }^{1} \mathrm{H} \mathrm{NMR}(700 \mathrm{MHz}$, $\left.\mathrm{CDCl}_{3}\right) \delta 6.62(\mathrm{~s}, 2 \mathrm{H}), 6.43(\mathrm{~s}, 1 \mathrm{H}), 5.31(\mathrm{~s}, 1 \mathrm{H}), 3.80(\mathrm{~s}, 6 \mathrm{H}), 3.34(\mathrm{~s}, 6 \mathrm{H}) ;{ }^{13} \mathrm{C} \mathrm{NMR}(176 \mathrm{MHz}$, $\left.\mathrm{CDCl}_{3}\right) \delta 160.8,140.5,104.5,103.1,100.7,55.4,52.8$; IR (neat) 2943, 2833, 1599, 1460, 1356, 1298; HRMS (ESI) m/e calcd for $\mathrm{C}_{11} \mathrm{H}_{16} \mathrm{O}_{4} \mathrm{Na}(\mathrm{M}+\mathrm{Na}) 235.0946$, found 235.0936.

To a solution of the diol $3 \mathbf{e}(100.0 \mathrm{mg}, 0.66 \mathrm{mmol})$ and the PPG reagent 7 (278.0 $\mathrm{mg}, 1.31$ $\mathrm{mmol})$ in DCM (2 mL) was added a catalytic amount of $p$-TsOH $(25 \mathrm{mg}, 0.13 \mathrm{mmol})$ at room temperature under $\mathrm{N}_{2}$. The reaction mixture was stirred for $7 \mathrm{~h}$. After completion of reaction, the reaction mixture was concentrated and purified with column chromatography (PE/EA 39:1) to produce $8(178 \mathrm{mg}, 90 \%)$ as a white solid. $\mathrm{Rf}=0.2(\mathrm{PE} / \mathrm{EA}=39: 1) ;{ }^{1} \mathrm{H} \mathrm{NMR}(700 \mathrm{MHz}$, $\left.\mathrm{CDCl}_{3}\right) \delta 7.36(\mathrm{t}, J=7.4 \mathrm{~Hz}, 2 \mathrm{H}), 7.30(\mathrm{t}, J=7.4 \mathrm{~Hz}, 1 \mathrm{H}), 7.24(\mathrm{~d}, J=7.5 \mathrm{~Hz}, 2 \mathrm{H}), 6.72(\mathrm{~s}, 2 \mathrm{H})$, $6.46(\mathrm{~s}, 1 \mathrm{H}), 5.53(\mathrm{~s}, 1 \mathrm{H}), 4.37(\mathrm{dd}, J=11.3,4.5 \mathrm{~Hz}, 2 \mathrm{H}), 4.04(\mathrm{t}, J=11.3 \mathrm{~Hz}, 1 \mathrm{H}), 3.82(\mathrm{~s}, 6 \mathrm{H})$, $3.38(\mathrm{~m}, 1 \mathrm{H}) ;{ }^{13} \mathrm{C} \mathrm{NMR}\left(176 \mathrm{MHz}, \mathrm{CDCl}_{3}\right) \delta 160.8,140.4,137.6,128.9,127.7,127.5,103.9$, 101.6, 101.4, 72.4, 55.4, 41.1; IR (neat) 3007, 2964, 2940, 2854, 1597, 1461, 1378,1201; HRMS (ESI) m/e calcd for $\mathrm{C}_{18} \mathrm{H}_{21} \mathrm{O}_{4}(\mathrm{M}+\mathrm{H})$ 301.1440, found 301.1435.

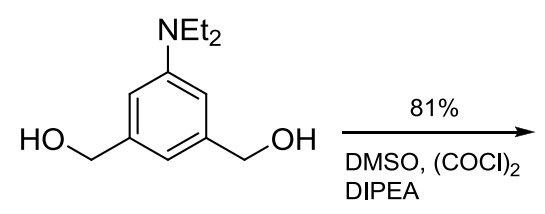

9

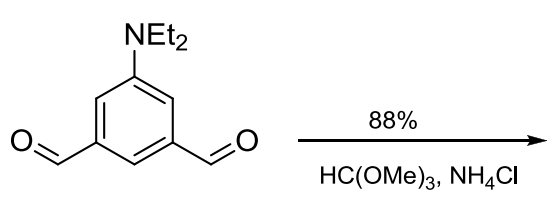

15

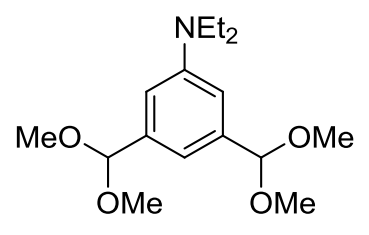

10 
To a stirred solution of oxalyl chloride $(0.68 \mathrm{~mL}, 7.5 \mathrm{mmol})$ in dry DCM $(50 \mathrm{~mL})$ at $-78{ }^{\circ} \mathrm{C}$ under nitrogen atmosphere, DMSO $(1.065 \mathrm{~mL}, 15 \mathrm{mmol})$ was added. The reaction mixture was stirred for 30 min before addition of the alcohol $9(0.523 \mathrm{~g}, 2.5 \mathrm{mmol})$. After stirring for $1 \mathrm{~h}$ at $78{ }^{\circ} \mathrm{C}$, DIPEA $(3.48 \mathrm{~mL}, 20 \mathrm{mmol})$ was added and the reaction mixture was stirred for another $30 \mathrm{~min}$. The reaction mixture was then gradually warmed up to $0{ }^{\circ} \mathrm{C}$ and was quenched with $\mathrm{H}_{2} \mathrm{O}$ $(25 \mathrm{~mL})$. The organic phase was separated and the aqueous phase was extracted with DCM $(1 \times 25 \mathrm{~mL})$. The combined organic phase was washed with water $(3 \times 15 \mathrm{~mL})$ and brine, dried over anhydrous $\mathrm{Na}_{2} \mathrm{SO}_{4}$, and concentrated under reduced pressure. The crude product was purified by column chromatography (PE/EA 30:1) to provide the desired dialdehyde $\mathbf{1 5}$ (418 $\mathrm{mg}, 81 \%)$ as a yellow solid. $\mathrm{Rf}=0.2\left(\mathrm{PE} / \mathrm{EA} \mathrm{30:1);}{ }^{1} \mathrm{H} \mathrm{NMR}\left(400 \mathrm{MHz}, \mathrm{CDCl}_{3}\right) \delta 10.01(\mathrm{~s}, 2 \mathrm{H}), 7.58(\mathrm{~s}, 1 \mathrm{H})\right.$, $7.37(\mathrm{~s}, 2 \mathrm{H}), 3.46(\mathrm{q}, J=7.1 \mathrm{~Hz}, 4 \mathrm{H}), 1.22(\mathrm{t}, J=7.1 \mathrm{~Hz}, 6 \mathrm{H}) ;{ }^{13} \mathrm{C} \mathrm{NMR}\left(176 \mathrm{MHz}, \mathrm{CDCl}_{3}\right) \delta$ 192.2, 148.6, 138.0, 119.4, 116.0, 44.6, 12.3; IR (neat) 2972, 2833, 1687, 1592; HRMS (ESI) $\mathrm{m} / \mathrm{e}$ calcd for $\mathrm{C}_{12} \mathrm{H}_{16} \mathrm{NO}_{2}(\mathrm{M}+\mathrm{H})$ 206.1181, found 206.1178 .

To a solution of the dialdehyde $(250 \mathrm{mg}, 1.22 \mathrm{mmol})$ and trimethylorthoformate $(1.1 \mathrm{~mL}, 9.736$ mmol) in methanol $(5 \mathrm{~mL})$ was added $\mathrm{NH}_{4} \mathrm{Cl}(13 \mathrm{mg}, 0.24 \mathrm{mmol})$ at room temperature under $\mathrm{N}_{2}$. The reaction mixture was refluxed and stirred for $24 \mathrm{~h}$. After completion, the reaction mixture was concentrated under reduced pressure. The residue was purified by column chromatography $(\mathrm{Hex} / \mathrm{Tol} / \mathrm{EA}$ 15:15:1) to provide the desired diacetal $10(319 \mathrm{mg}, 88 \%)$ as a yellow solid. $\mathrm{Rf}=$ $0.2(\mathrm{Hex} / \mathrm{Tol} / \mathrm{EA} 15: 15: 1) ;{ }^{1} \mathrm{H} \mathrm{NMR}\left(400 \mathrm{MHz}, \mathrm{CDCl}_{3}\right) \delta 6.79(\mathrm{~s}, 1 \mathrm{H}), 6.73(\mathrm{~s}, 2 \mathrm{H}), 5.29(\mathrm{~s}, 2 \mathrm{H})$, 3.40-3.34 (m, 16H), 1.14 (t, $J=7.0 \mathrm{~Hz}, 6 \mathrm{H}) ;{ }^{13} \mathrm{C} \mathrm{NMR}\left(176 \mathrm{MHz}, \mathrm{CDCl}_{3}\right) \delta 147.9,138.9,112.6$, 110.0, 103.8, 52.9, 44.3, 12.6; IR (neat) 2967, 2933, 2894, 2827, 1602, 1465; HRMS (ESI) m/e calcd for $\mathrm{C}_{16} \mathrm{H}_{28} \mathrm{NO}_{4}(\mathrm{M}+\mathrm{H})$ 298.2018, found 298.2020.
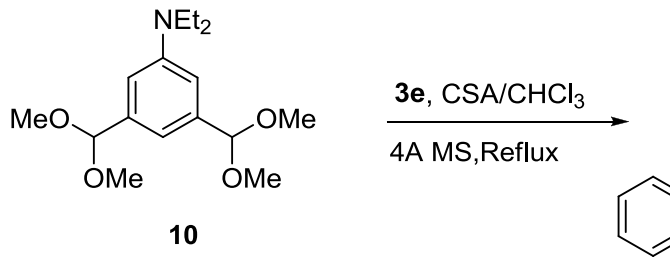

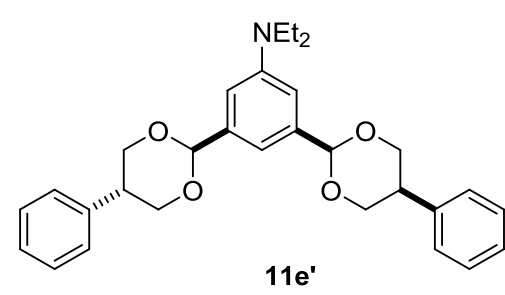

$11 e^{\prime}$

To a solution of diol 3e (202 $\mathrm{mg}, 1.33 \mathrm{mmol})$ and the PPG reagent $10(158 \mathrm{mg}, 0.53 \mathrm{mmol})$ in $\mathrm{CH}_{3} \mathrm{Cl}(3 \mathrm{~mL})$ was added a catalytic amount of CSA $(25 \mathrm{mg}, 0.11 \mathrm{mmol})$ and $4 \mathrm{~A} \mathrm{MS}(25 \mathrm{mg})$ at room temperature under $\mathrm{N}_{2}$. The reaction mixture was refluxed for $3 \mathrm{~h}$. After completion of reaction, the reaction solution was neutralized with TEA at room temperature and then concentrated under reduced pressure. The crude product was purified by column chromatography (PE/EA 30:1 to 10:1) to provide the pure diacetal 11e (95 mg), 11e' $(62 \mathrm{mg})$, and a mixture of $11 \mathrm{e}$ and $11 \mathrm{e}^{\prime}(58 \mathrm{mg})$ in a combined $86 \%$ yield.

For 11e, $\mathrm{Rf}=0.3(\mathrm{PE} / \mathrm{EA} 10: 1) ;{ }^{1} \mathrm{H}$ NMR $\left(700 \mathrm{MHz}, \mathrm{CDCl}_{3}\right) \delta 7.35(\mathrm{t}, J=7.5 \mathrm{~Hz}, 4 \mathrm{H}), 7.28(\mathrm{t}, J$ $=7.3 \mathrm{~Hz}, 2 \mathrm{H}), 7.24(\mathrm{~d}, J=7.2 \mathrm{~Hz}, 4 \mathrm{H}), 6.99(\mathrm{~s}, 1 \mathrm{H}), 6.85(\mathrm{~s}, 2 \mathrm{H}), 5.54(\mathrm{~s}, 2 \mathrm{H}), 4.36(\mathrm{dd}, J=$ $11.5,4.6 \mathrm{~Hz}, 4 \mathrm{H}), 4.02(\mathrm{t}, J=11.5 \mathrm{~Hz}, 4 \mathrm{H}), 3.41-3.36(\mathrm{~m}, 6 \mathrm{H}), 1.17(\mathrm{t}, J=7.0 \mathrm{~Hz}, 6 \mathrm{H}) ;{ }^{13} \mathrm{C}$ NMR $\left(176 \mathrm{MHz}, \mathrm{CDCl}_{3}\right) \delta 148.1,139.3,137.9,128.8,127.7,111.2,110.0,101.9,72.3,44.2$, 
41.1, 12.7; IR (neat) 2966, 2921, 2872, 1598,1488, 1089; HRMS (ESI) m/e calcd for $\mathrm{C}_{30} \mathrm{H}_{36} \mathrm{NO}_{4}$ (M+H) 474.2644, found 474.2635.

For 11e', Rf $=0.28(\mathrm{PE} / \mathrm{EA} 10: 1) ;{ }^{1} \mathrm{H}$ NMR $\left(700 \mathrm{MHz}, \mathrm{CDCl}_{3}\right) \delta 7.64(\mathrm{~d}, J=7.0 \mathrm{~Hz}, 2 \mathrm{H}), 7.37$ (t, $J=7.5 \mathrm{~Hz}, 2 \mathrm{H}), 7.34(\mathrm{t}, J=7.8 \mathrm{~Hz}, 2 \mathrm{H}), 7.31(\mathrm{t}, J=7.5 \mathrm{~Hz}, 1 \mathrm{H}), 7.26(\mathrm{~d}, J=7.5 \mathrm{~Hz}, 3 \mathrm{H})$, $6.97(\mathrm{~s}, 1 \mathrm{H}), 6.90(\mathrm{~s}, 1 \mathrm{H}), 6.87(\mathrm{~s}, 1 \mathrm{H}), 5.68(\mathrm{~s}, 1 \mathrm{H}), 5.56(\mathrm{~s}, 1 \mathrm{H}), 4.39-4.36(\mathrm{~m}, 6 \mathrm{H}), 4.04(\mathrm{t}, J=$ $11.0 \mathrm{~Hz}, 2 \mathrm{H}), 3.43-3.40(\mathrm{~m}, 5 \mathrm{H}), 1.19(\mathrm{t}, J=7.0 \mathrm{~Hz}, 6 \mathrm{H}) ;{ }^{13} \mathrm{C} \mathrm{NMR}\left(176 \mathrm{MHz}, \mathrm{CDCl}_{3}\right) \delta 148.1$, $142.9,139.5,139.2,137.9,128.8,128.6,128.3,127.7,127.4,126.5,111.4,110.1,109.8,102.0$, 101.7, 72.4, 70.9, 44.4, 41.2, 39.6, 12.7; IR (neat) 2968, 2922, 2865, 1600, 1488, 1404; HRMS (ESI) $\mathrm{m} / \mathrm{e}$ calcd for $\mathrm{C}_{30} \mathrm{H}_{36} \mathrm{NO}_{4}(\mathrm{M}+\mathrm{H}) 474.2644$, found 474.2633 .

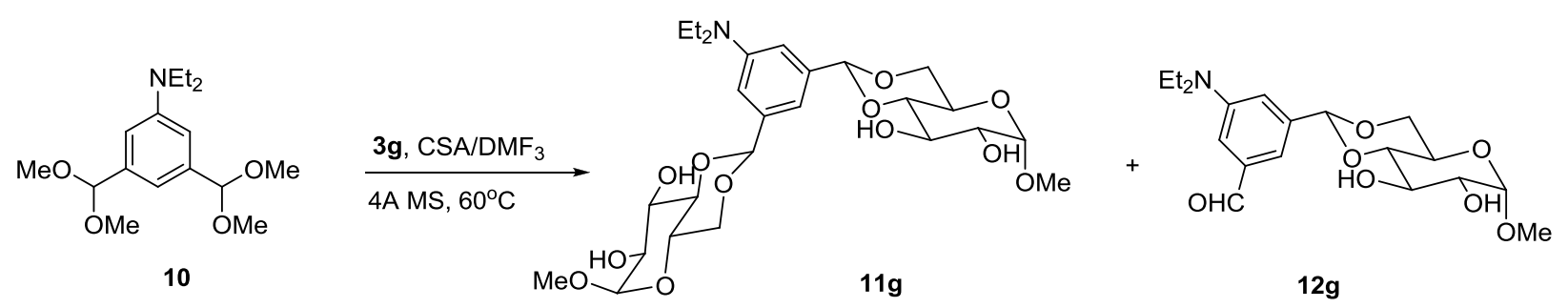

To a solution of $\mathbf{3 g}(158 \mathrm{mg}, 0.81 \mathrm{mmol})$ and the PPG reagent 10 (97 $\mathrm{mg}, 0.33 \mathrm{mmol})$ in DMF (3 $\mathrm{mL})$ was added a catalytic amount of CSA $(15 \mathrm{mg}, 0.065 \mathrm{mmol})$ and $4 \mathrm{~A}$ molecular sieve $(25 \mathrm{mg})$ at room temperature under $\mathrm{N}_{2}$. The reaction mixture was heated at $60^{\circ} \mathrm{C}$ for $4 \mathrm{~h}$. After completion, the reaction mixture was neutralized with TEA and directly purified with column chromatography (DCM/MeOH 50:1) to provide $\mathbf{1 1 g}$ (66 mg, 29\%) and $\mathbf{1 2 g}(54 \mathrm{mg}, 35 \%$ ).

For 11g, $\quad \mathrm{Rf}=0.25(\mathrm{DCM} / \mathrm{MeOH} 50: 1) ;{ }^{1} \mathrm{H}$ NMR $\left(700 \mathrm{MHz}, \mathrm{CDCl}_{3} / \mathrm{CD}_{3} \mathrm{CN} 5: 2\right) \delta 6.85(\mathrm{~s}$, $1 \mathrm{H}), 6.74(\mathrm{~s}, 2 \mathrm{H}), 5.44(\mathrm{~s}, 2 \mathrm{H}), 4.74(\mathrm{~d}, J=3.8 \mathrm{~Hz}, 2 \mathrm{H}) ; 4.22$ (dd, $J=10.2,4.8 \mathrm{~Hz}, 2 \mathrm{H}) ; 3.80$ (td, $J=9.1,3.5 \mathrm{~Hz}, 2 \mathrm{H}) ; 3.74(\mathrm{td}, J=9.8,4.2 \mathrm{~Hz}, 2 \mathrm{H}) ; 3.69(\mathrm{t}, J=9.2 \mathrm{~Hz}, 2 \mathrm{H}) ; 3.53(\mathrm{td}, J=9.2,4.2$ $\mathrm{Hz}, 2 \mathrm{H}) ; 3.43$ (s, 6H), 3.42 (t, $J=9.1 \mathrm{~Hz}, 2 \mathrm{H}) ; 3.38-3.35(\mathrm{~m}, 6 \mathrm{H}), 2.88(\mathrm{~d}, J=8.4 \mathrm{~Hz}, 2 \mathrm{H}) ; 1.13$ $(\mathrm{t}, J=7.0 \mathrm{~Hz}, 3 \mathrm{H}) ;{ }^{13} \mathrm{C}$ NMR $\left(700 \mathrm{MHz}, \mathrm{CDCl}_{3} / \mathrm{CD}_{3} \mathrm{CN} 5: 2\right) \delta 152.9,143.9,116.8,115.8$, 107.54, 107.52, 105.5, 86.5, 78.2, 76.4, 74.1, 67.8, 60.6, 49.5, 17.6; IR (neat) 3347, 2926, 2867, 1606, 1364; HRMS (ESI) m/e calcd for $\mathrm{C}_{26} \mathrm{H}_{40} \mathrm{NO}_{12}(\mathrm{M}+\mathrm{H})$ 558.2551, found 558.2548.

For 12g, $\quad \mathrm{Rf}=0.35(\mathrm{DCM} / \mathrm{MeOH} 15: 1) ;{ }^{1} \mathrm{H}$ NMR $\left(400 \mathrm{MHz}, \mathrm{CDCl}_{3}\right) \delta 9.92(\mathrm{~s}, 1 \mathrm{H}), 7.27(\mathrm{~s}$, 1H), $7.12(\mathrm{~s}, 1 \mathrm{H}), 7.00(\mathrm{~s}, 1 \mathrm{H}), 5.52(\mathrm{~s}, 1 \mathrm{H}), 4.81(\mathrm{~s}, 1 \mathrm{H}), 4.31(\mathrm{dd}, J=10.1,4.8 \mathrm{~Hz}, 1 \mathrm{H}) ; 3.94(\mathrm{t}$, $J=8.2 \mathrm{~Hz}, 1 \mathrm{H}) ; 3.83(\mathrm{~m}, 1 \mathrm{H}), 3.75(\mathrm{t}, J=10.3 \mathrm{~Hz}, 1 \mathrm{H}) ; 3.64(\mathrm{~m}, 1 \mathrm{H}), 3.51(\mathrm{t}, J=9.4 \mathrm{~Hz}, 1 \mathrm{H})$; $3.47(\mathrm{~s}, 3 \mathrm{H}), 3.44-3.36(\mathrm{~m}, 5 \mathrm{H}), 2.88(\mathrm{~s}, 1 \mathrm{H}), 2.39(\mathrm{~d}, J=14 \mathrm{~Hz}, 1 \mathrm{H}) ; 1.69(\mathrm{~s}, 1 \mathrm{H}) ; 1.17(\mathrm{t}, J=$ $7.0 \mathrm{~Hz}, 3 \mathrm{H}) ;{ }^{13} \mathrm{C}$ NMR $\left(700 \mathrm{MHz}, \mathrm{CDCl}_{3}\right) \delta 193.1,148.2,139.0,137.6,116.0,115.5,111.5$, 101.8, 99.8, 81.0, 72.9, 71.8, 69.0, 62.3, 55.7, 44.4, 12.6; IR (neat) 3385, 3246, 2935, 2869, 1699, 1600, 1075; HRMS (ESI) m/e calcd for $\mathrm{C}_{19} \mathrm{H}_{28} \mathrm{NO}_{7}(\mathrm{M}+\mathrm{H}) 382.1866$, found 382.1859 . 
General procedure of photoreaction in NMR tubes: A $5.0 \mathrm{mM}$ solution of $\mathbf{1 g}$ ( $39 \mathrm{mg}$ in 24 $\mathrm{mL}$ of $\mathrm{MeOH}$ ) was equally distributed into 16 of $5 \mathrm{~mm}$ NMR tubes (1.5 $\mathrm{mL} \times 16)$ in order to be irradiated under the same optimal conditions. (Typically, we first conducted photoreactions in NMR tubes to optimize the reaction conditions because we use ${ }^{1} \mathrm{H}$ NMR to monitor the reactions. Photo-deprotection reactions can also be carried out in other reaction vessels, for example, in a $250 \mathrm{~mL}$ reaction vessel in the regular setting of a Hanovia photoreactor. ${ }^{[1]}$ ) The NMR tubes were sealed and bound to the immersion well condenser of a Hanovia photoreactor with a $450 \mathrm{~W}$ medium pressure UV lamp. The reaction solution was irradiated with the UV light filtered through a Pyrex filter sleeve for $30 \mathrm{~min}$. The reaction solutions in all NMR tubes were combined, concentrated and purified with column chromatography (DCM/MeOH 15:1 to 10:1) to provide the methyl glucoside $\mathbf{3 g}$ ( $22 \mathrm{mg}$, 94\%).

For the photo deprotection reaction of $\mathbf{1 b}$, the product $\mathbf{3 b}$ was derivatized with $\mathrm{TBSOTf} / \mathrm{Et}_{3} \mathrm{~N}$, and isolated as the corresponding TBS-protected diol $\mathbf{S 1}$ in $81 \%$ yield. $\mathrm{Rf}=0.80(\mathrm{PE} / \mathrm{EA} 19: 1) ;{ }^{1} \mathrm{H}$ NMR $\left(400 \mathrm{MHz}, \mathrm{CDCl}_{3}\right) \delta 5.81(\mathrm{~m}, 1 \mathrm{H}), 5.18(\mathrm{dd}, J=17.2,1.6 \mathrm{~Hz}, 1 \mathrm{H}), 5.08(\mathrm{dd}, J=10.4,1.4 \mathrm{~Hz}$, $1 \mathrm{H}), 3.91(\mathrm{~m}, 2 \mathrm{H}), 3.75(\mathrm{~m}, 1 \mathrm{H}), 3.50-3.41(\mathrm{~m}, 3 \mathrm{H}), 3.30(\mathrm{~m}, 1 \mathrm{H}), 0.8(\mathrm{~s}, 18 \mathrm{H}),-0.03(\mathrm{~s}, 12 \mathrm{H})$; ${ }^{13} \mathrm{C}$ NMR $\left(75 \mathrm{MHz}, \mathrm{CDCl}_{3}\right) \delta 135.0,116.5,72.7,72.3,65.1,25.9,18.3,18.2$; IR (neat) 2954, 2929, 2885, 2857, 1472, 1253; HRMS (ESI) m/e calcd for $\mathrm{C}_{18} \mathrm{H}_{41} \mathrm{O}_{3} \mathrm{Si}_{2}(\mathrm{M}+\mathrm{H}) 361.2594$, found 361.2594 .

Quantum yield determination: A $5.0 \mathrm{mM}$ solution of $\mathbf{1}$ (in methanol) and a $5.0 \mathrm{mM}$ solution of DEABnprotected ethylene glycol monoallyl ether (with known quantum yield of 0.26$)^{[2]}\left(\mathrm{CD}_{3} \mathrm{CN}_{2} / \mathrm{D}_{2} \mathrm{O} 4: 1\right.$ ) in NMR tube were placed behind a standard $1 \mathrm{~cm}$ quartz UV cuvette contain the filter solution. Filtered light centered at $312 \mathrm{~nm}$ was obtained by passing light from the $450 \mathrm{~W}$ medium pressure mercury lamp through a solution of $2.0 \mathrm{mM} \mathrm{K} \mathrm{CrO}_{4}$ in a $5 \% \mathrm{~K}_{2} \mathrm{CO}_{3}$ aqueous solution. ${ }^{[3]}$ The yields of photo reactions were determined by ${ }^{1} \mathrm{H}$ NMR analysis.

\section{References:}

[1] Wang, P.; Devalankar, D.; Lu, W. J. Org. Chem. 2016, 81, 6195.

[2] Wang, P.; Lu, W.; Devalankar, D. A.; Ding, Z. Org. Lett. 2015, 17, 2114.

[3] Filter solutions: (a) Zimmerman, H.; Nuss, J.; Tantillo, A. J. Org. Chem. 1988, 53, 3792. (b) Zimmerman, H. Mol. Photochem. 1971, 3, 281. 


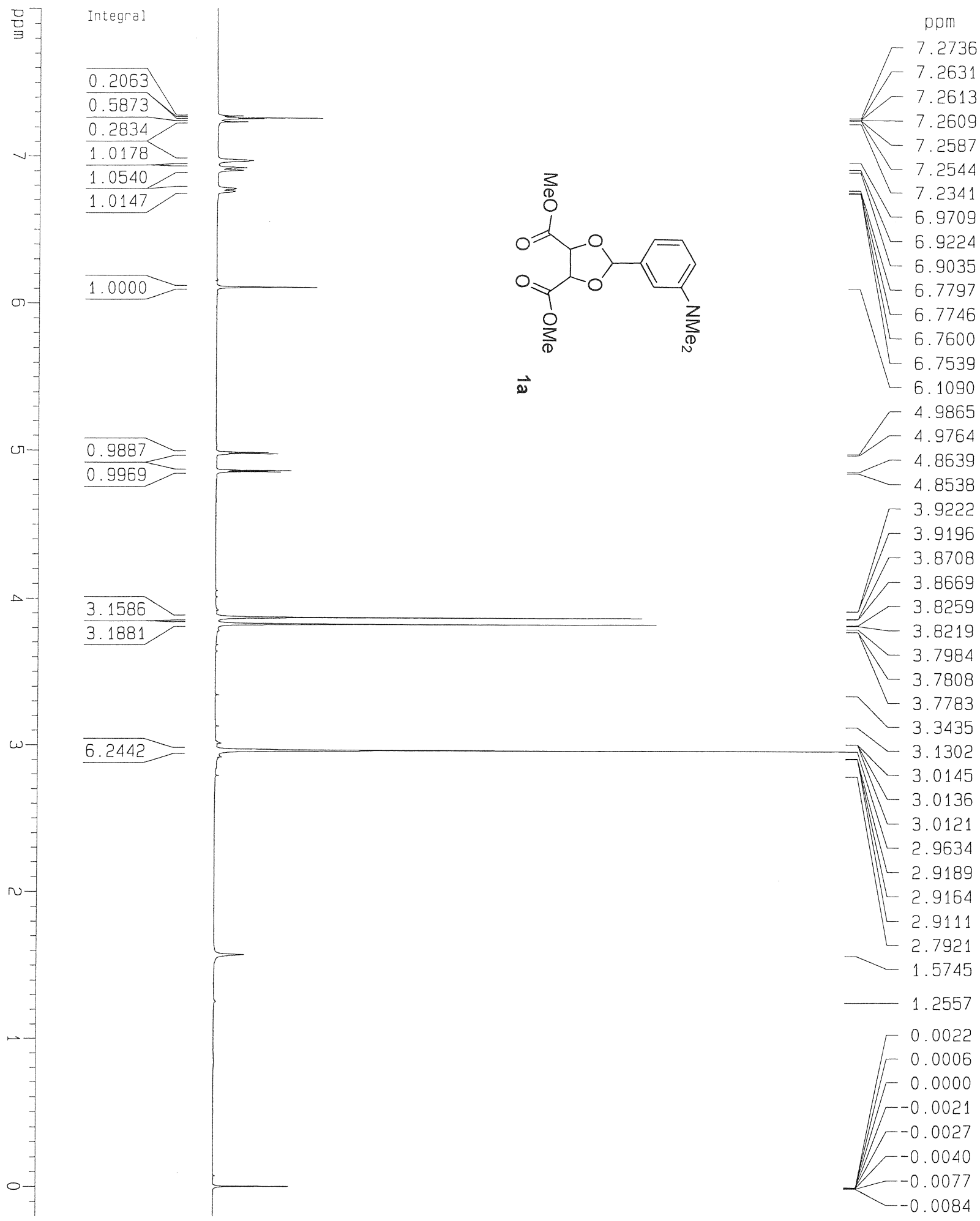

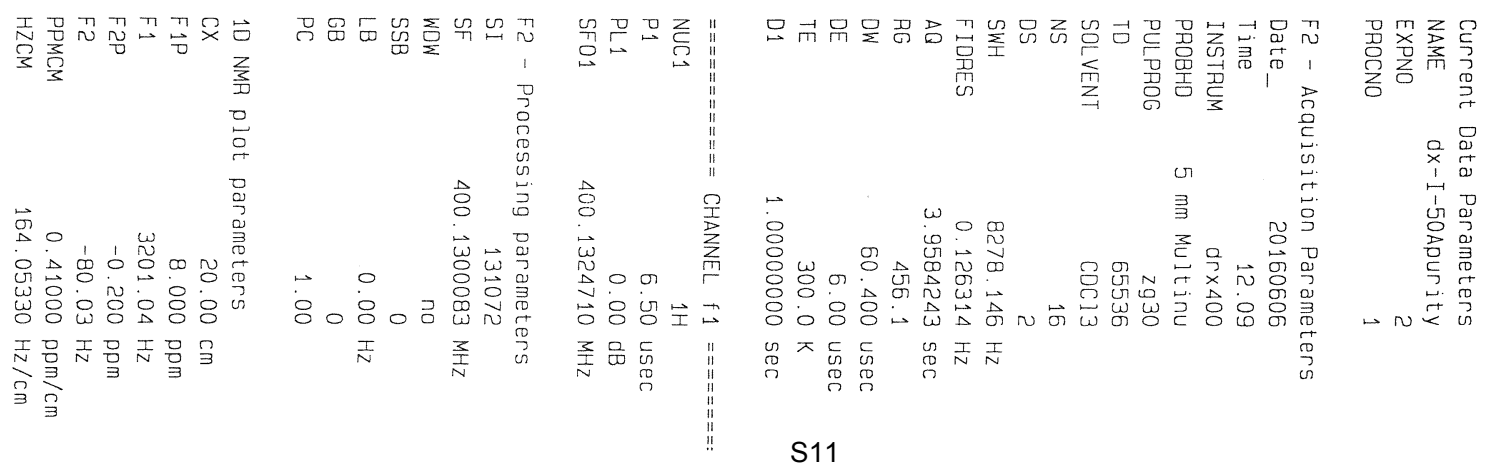




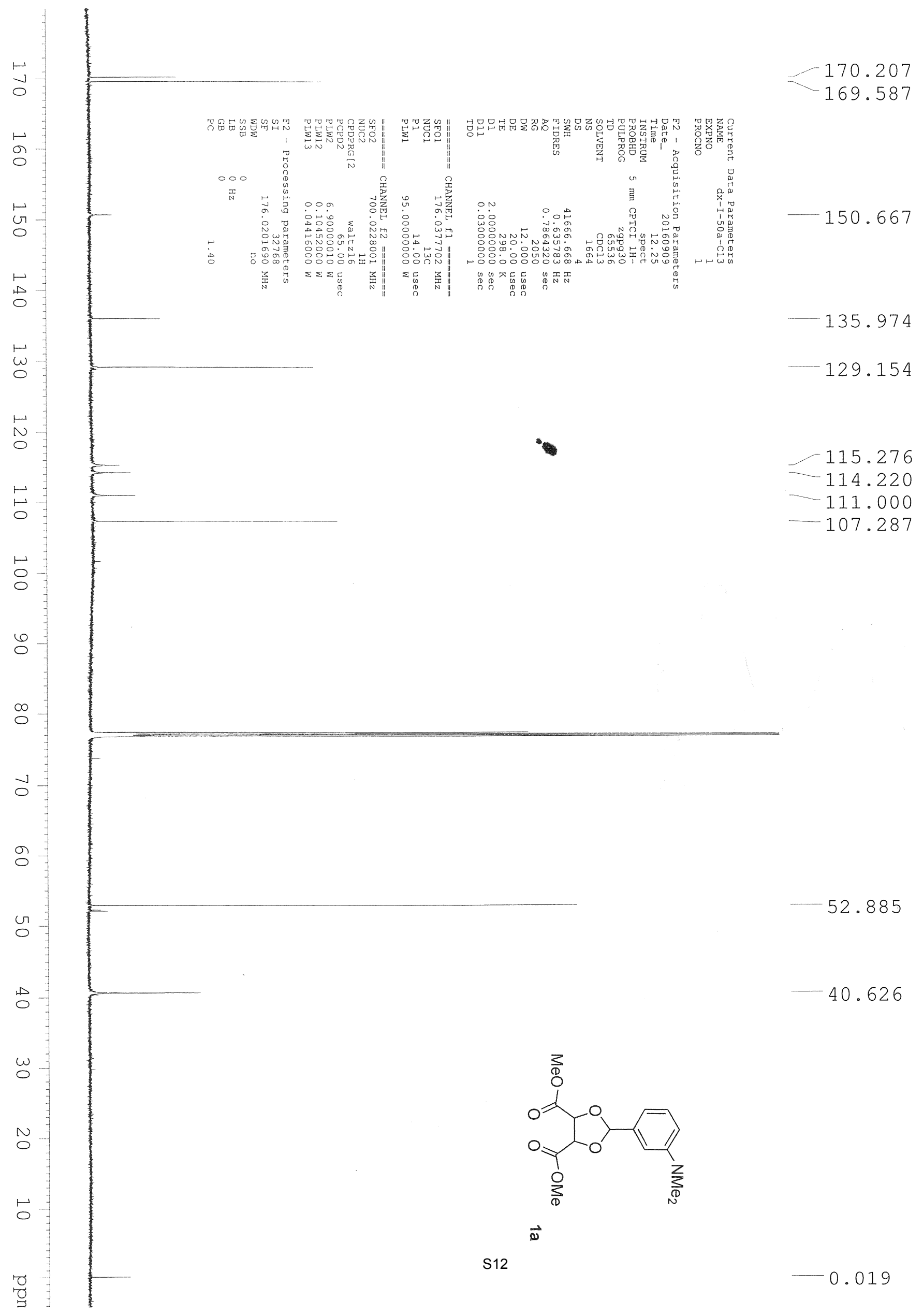




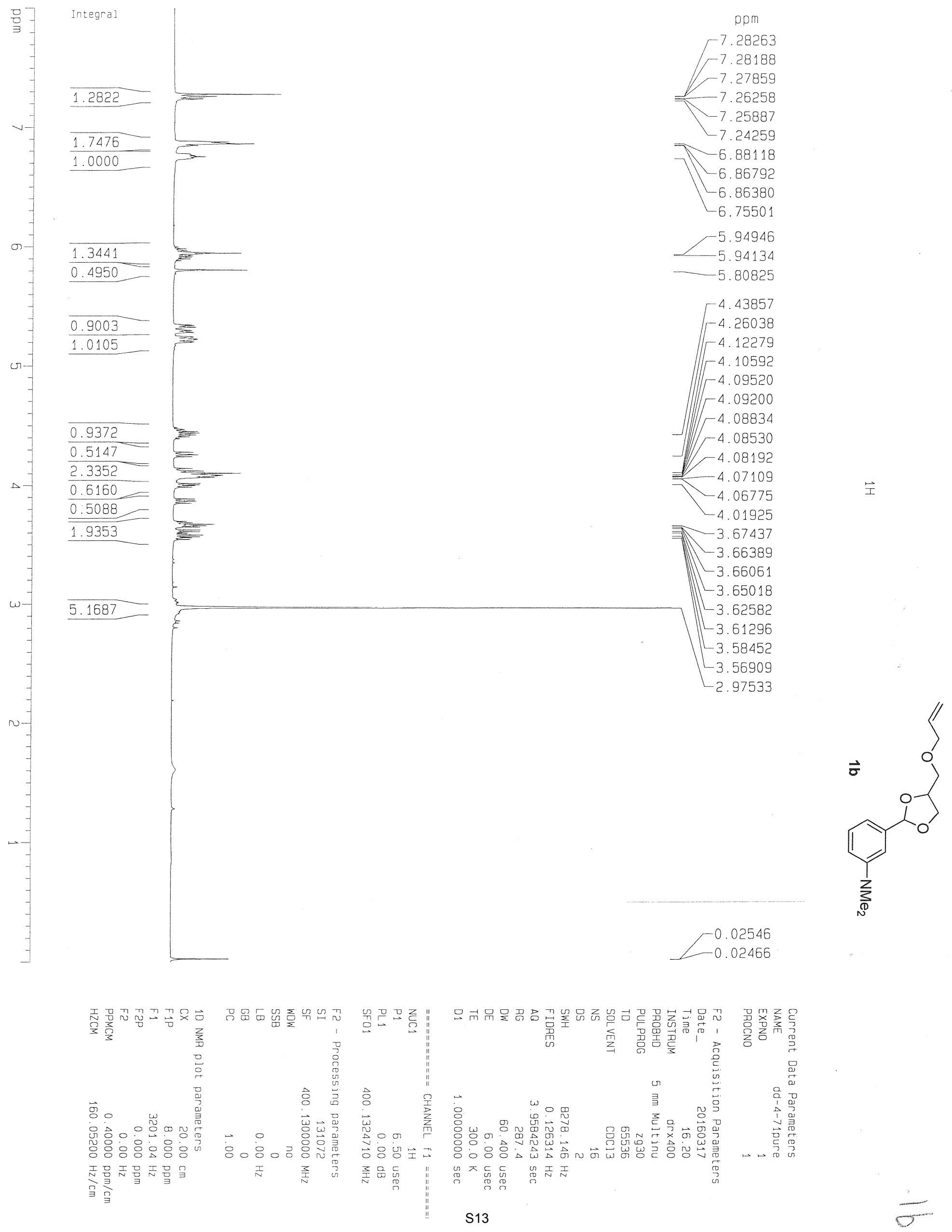



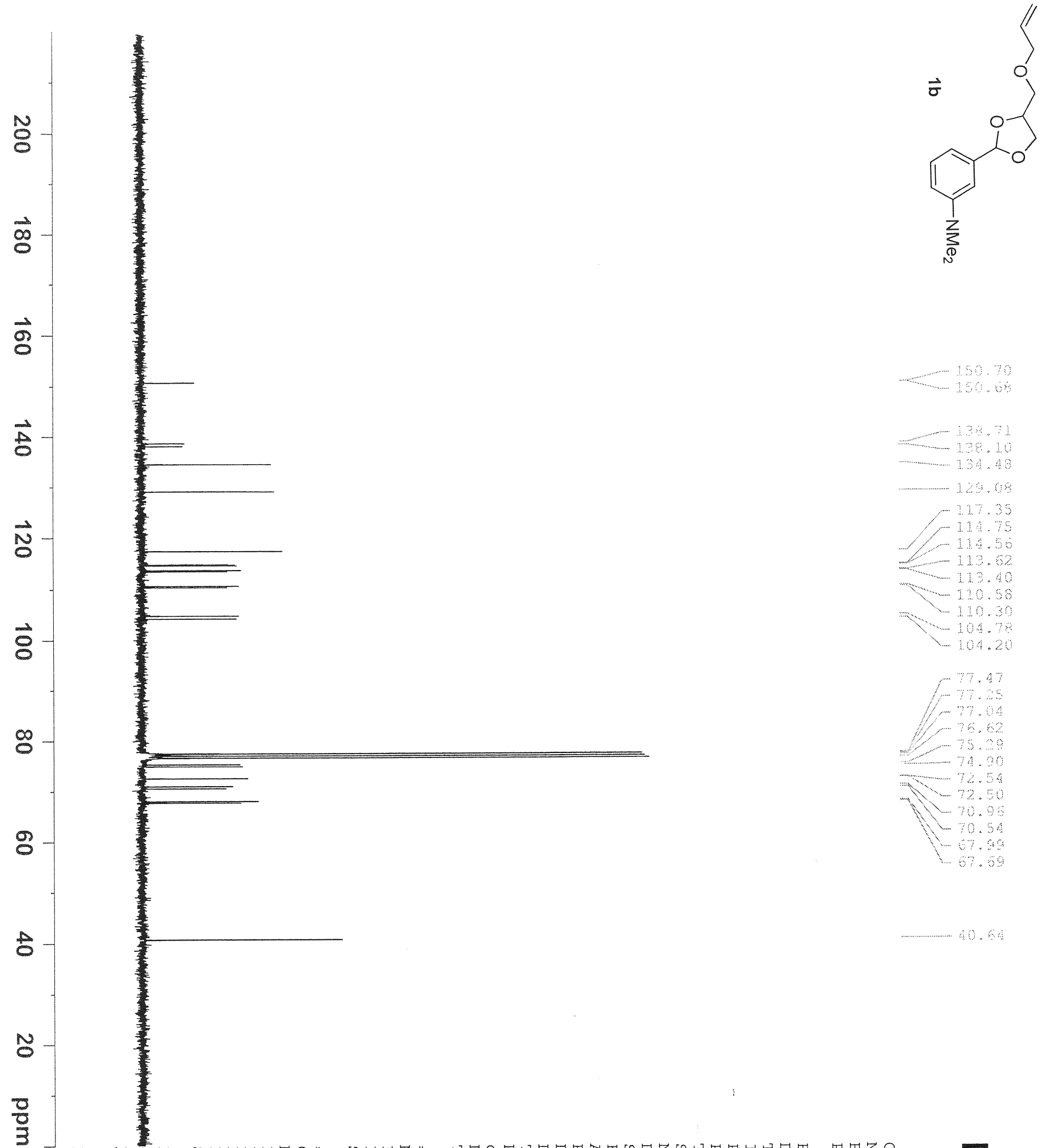

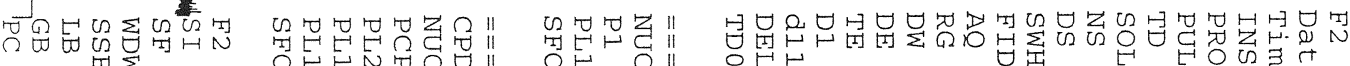

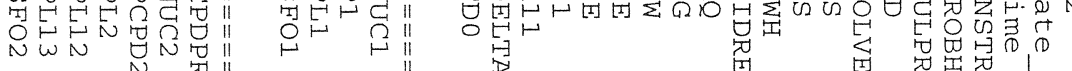
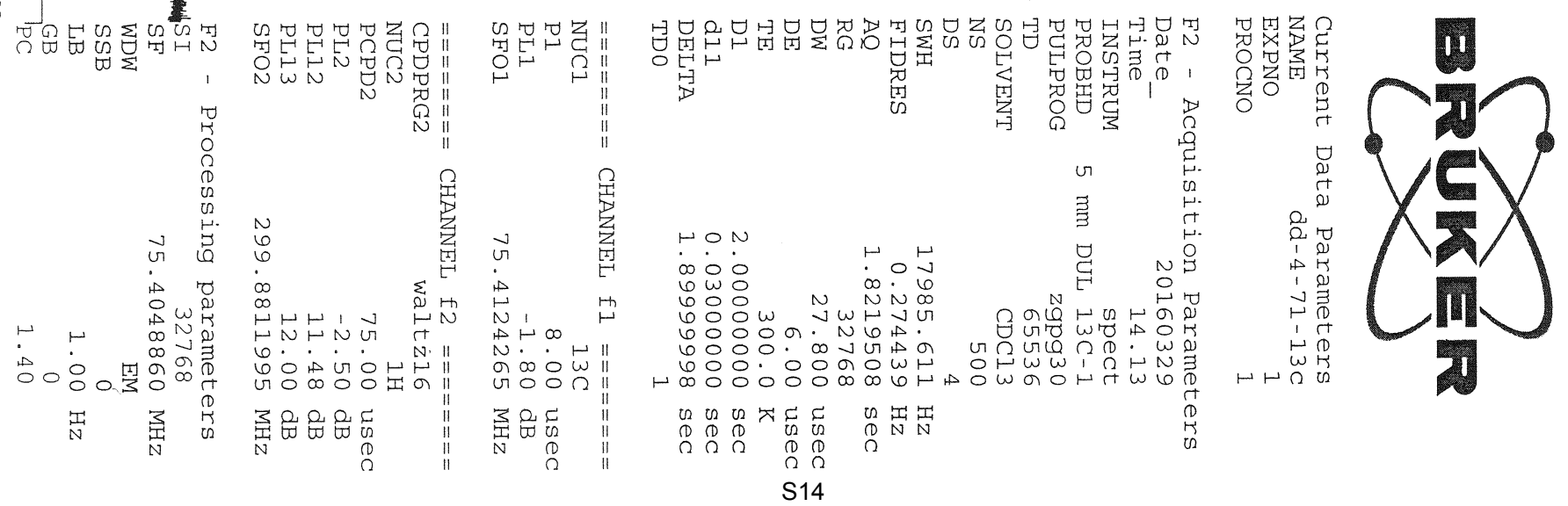


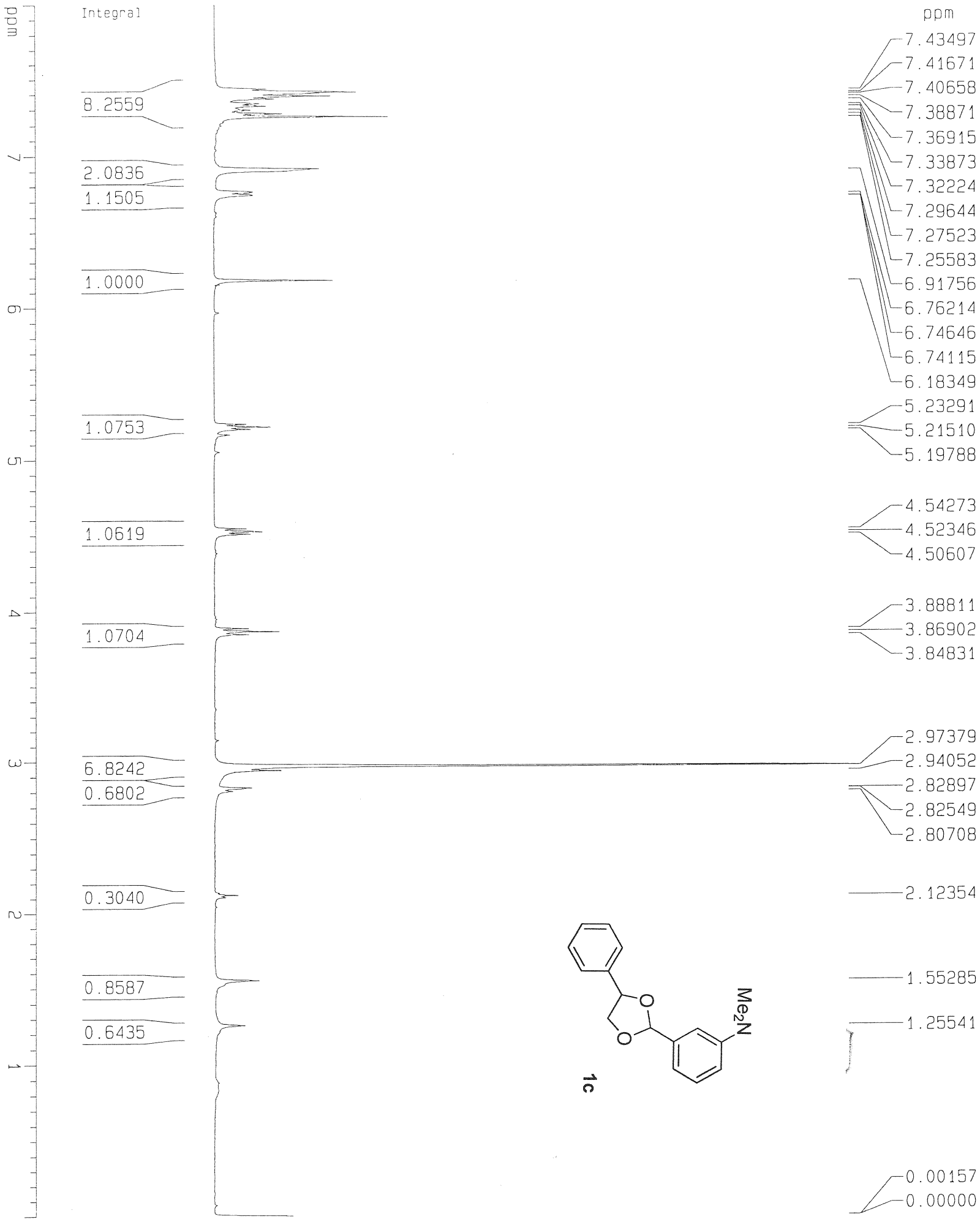

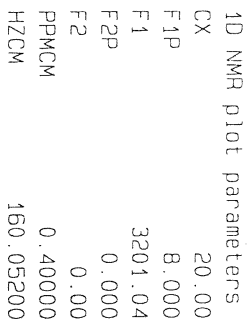

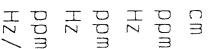
高旁

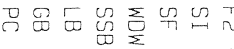

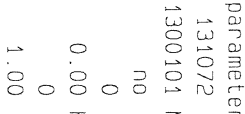
늄뭄몰

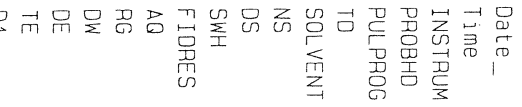

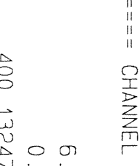
苛ㅇㅇㅇ요ㅇㅗㅗ

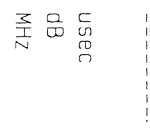
폰 좃

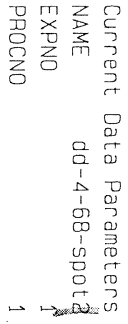

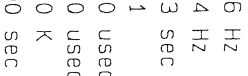

$\frac{\frac{8.2559}{1.0836}}{\frac{1.0000}{1.0753}}$ 


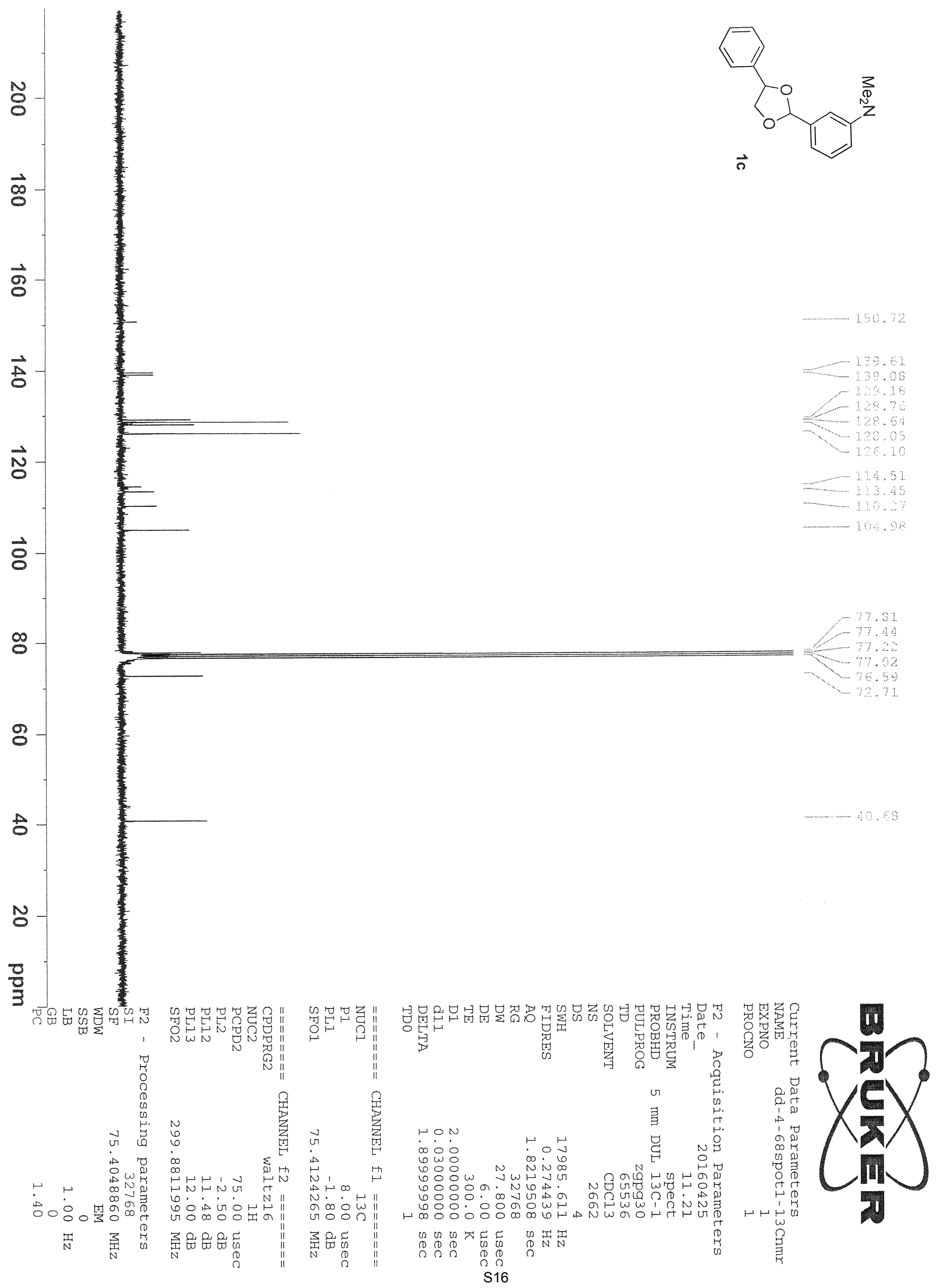




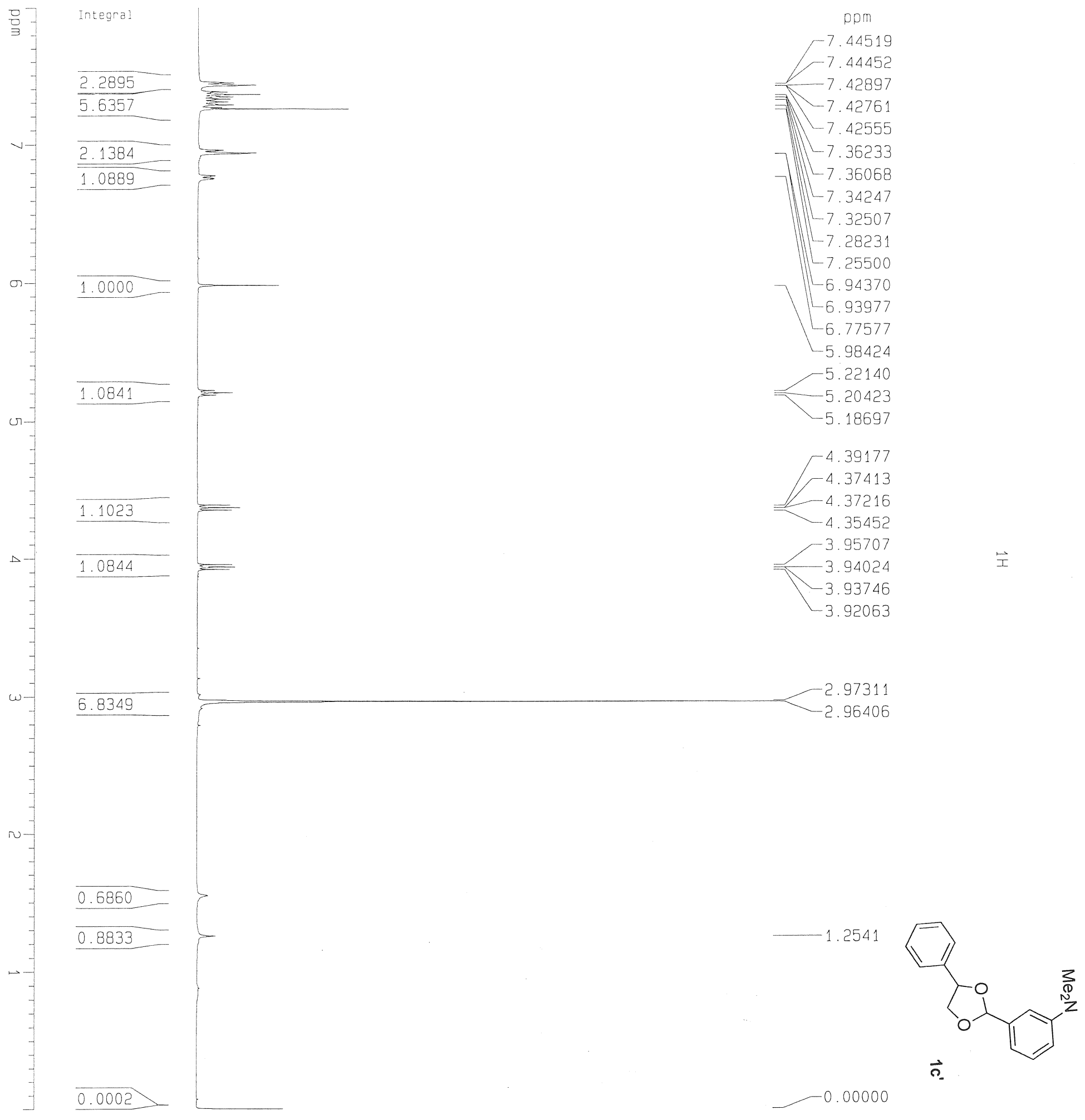

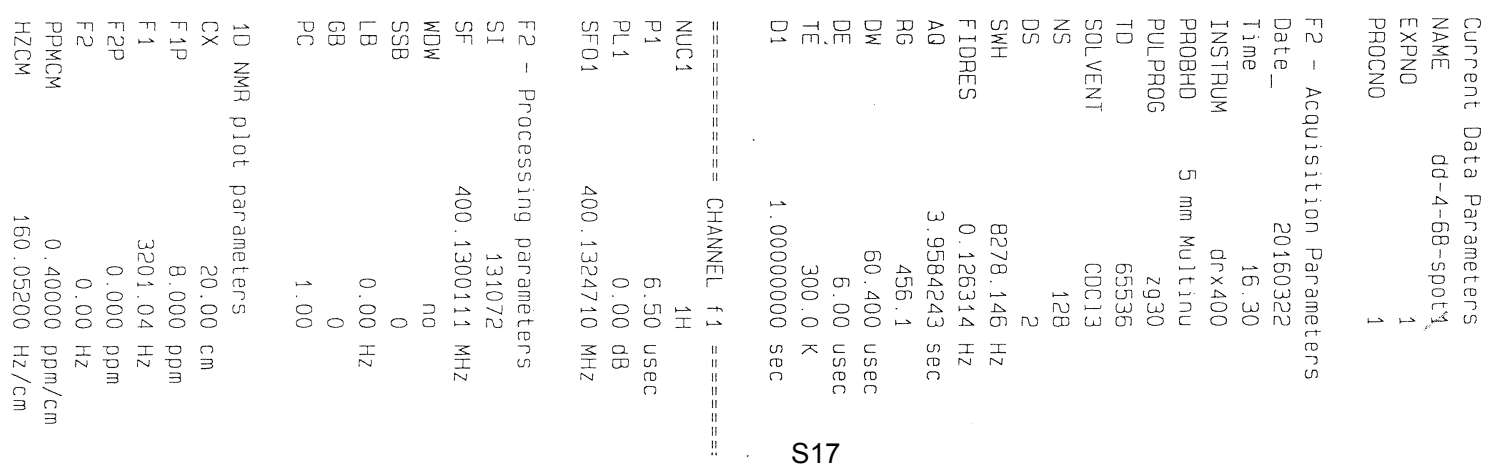




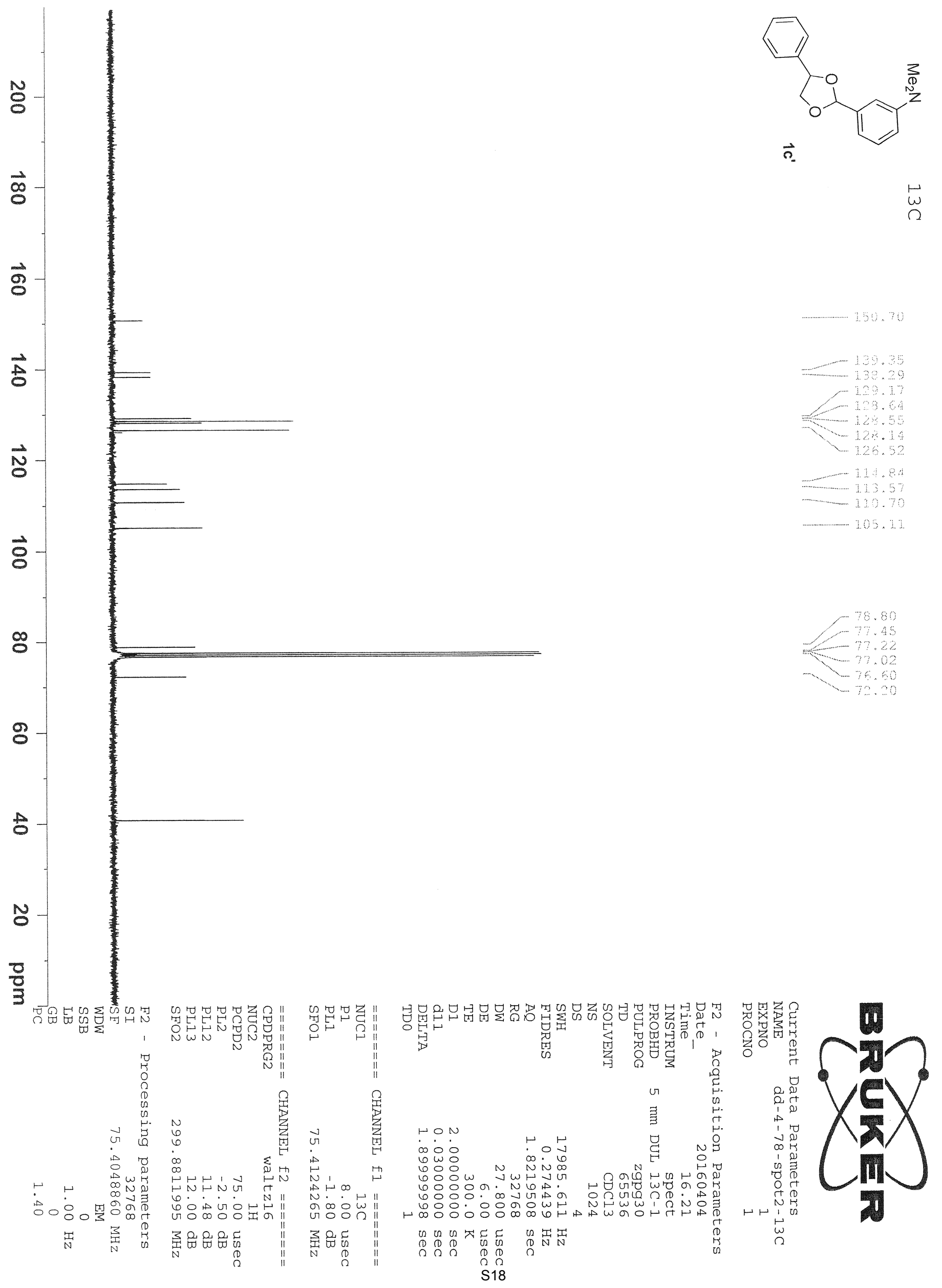



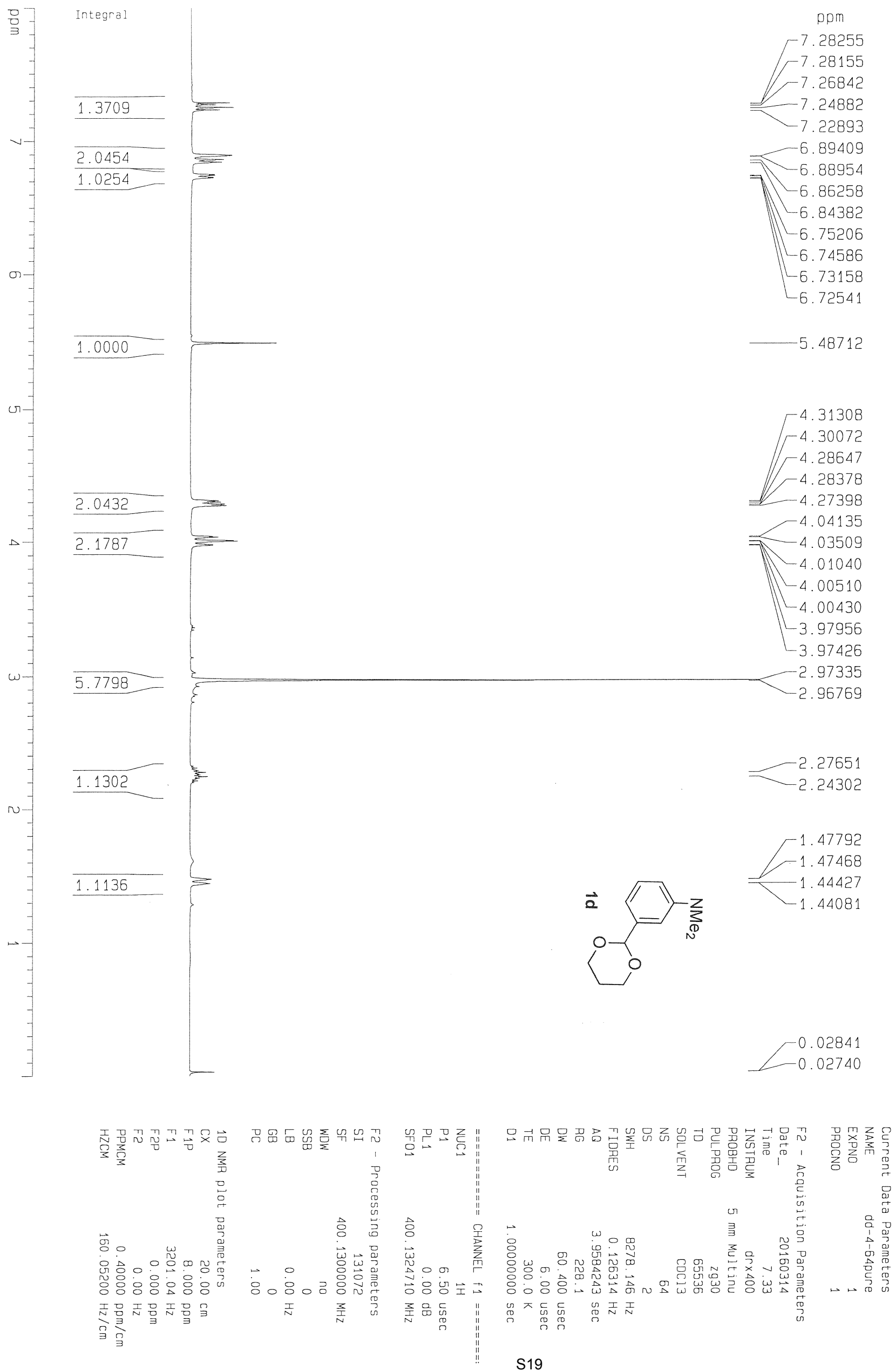


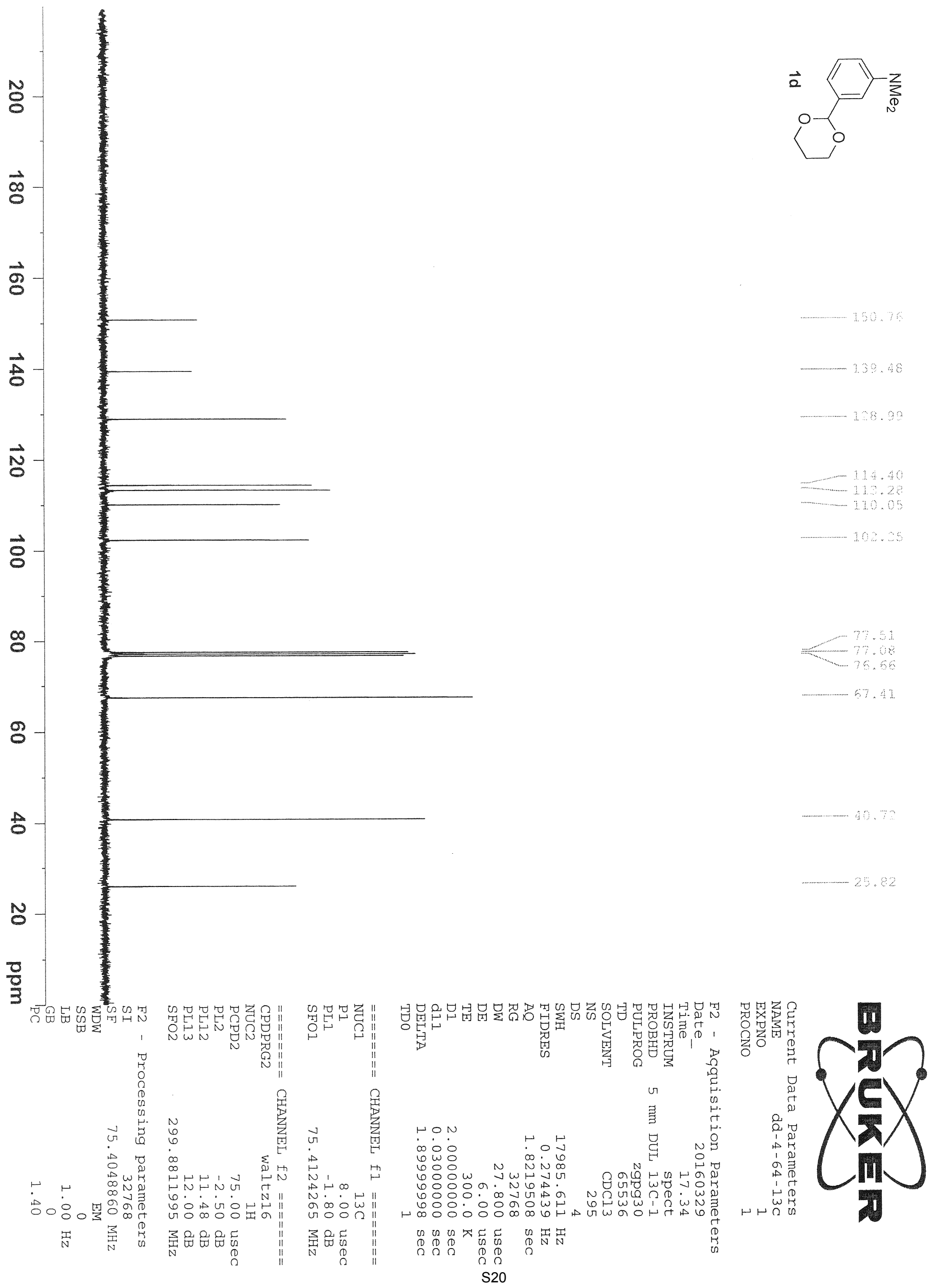



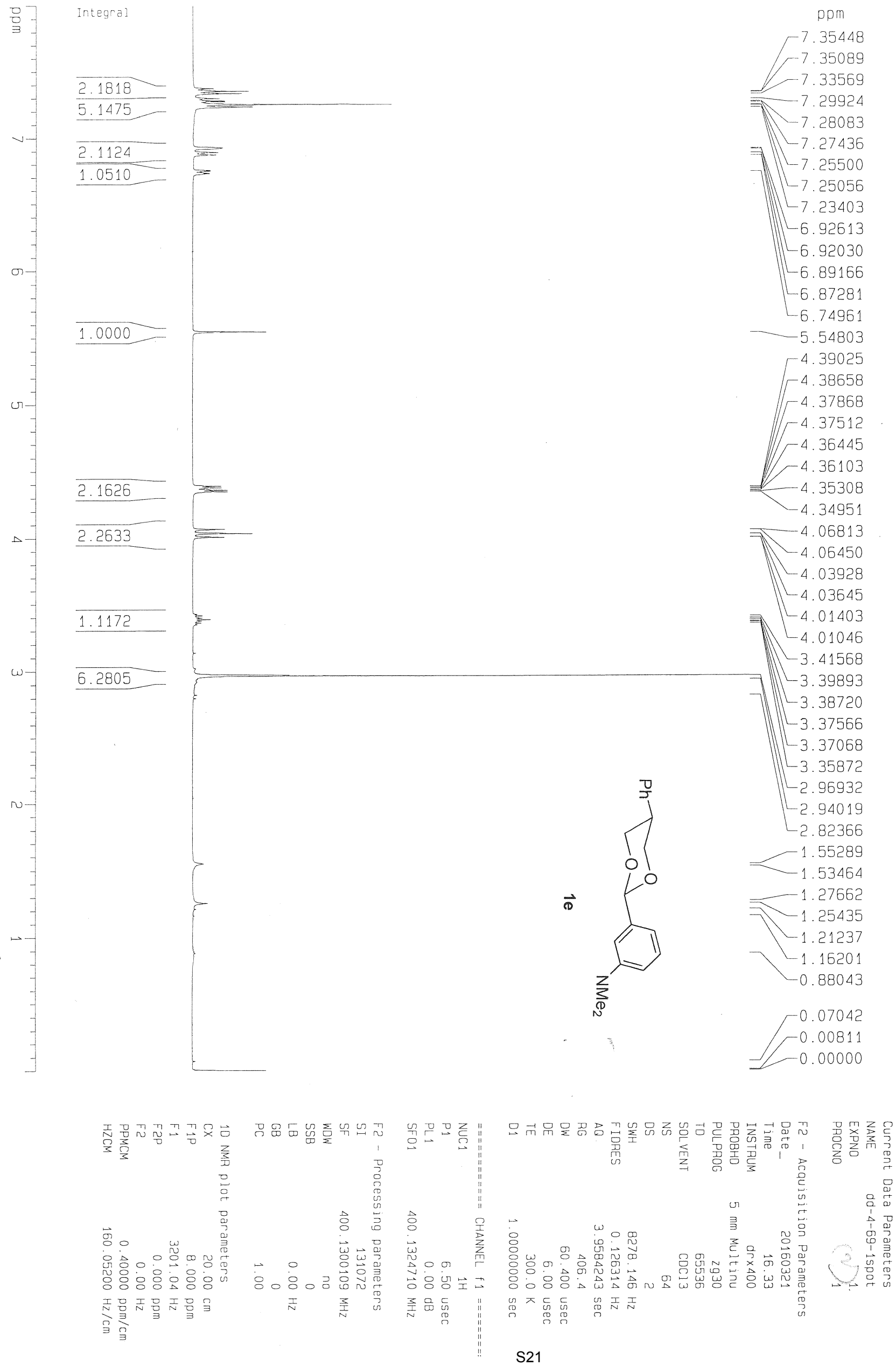


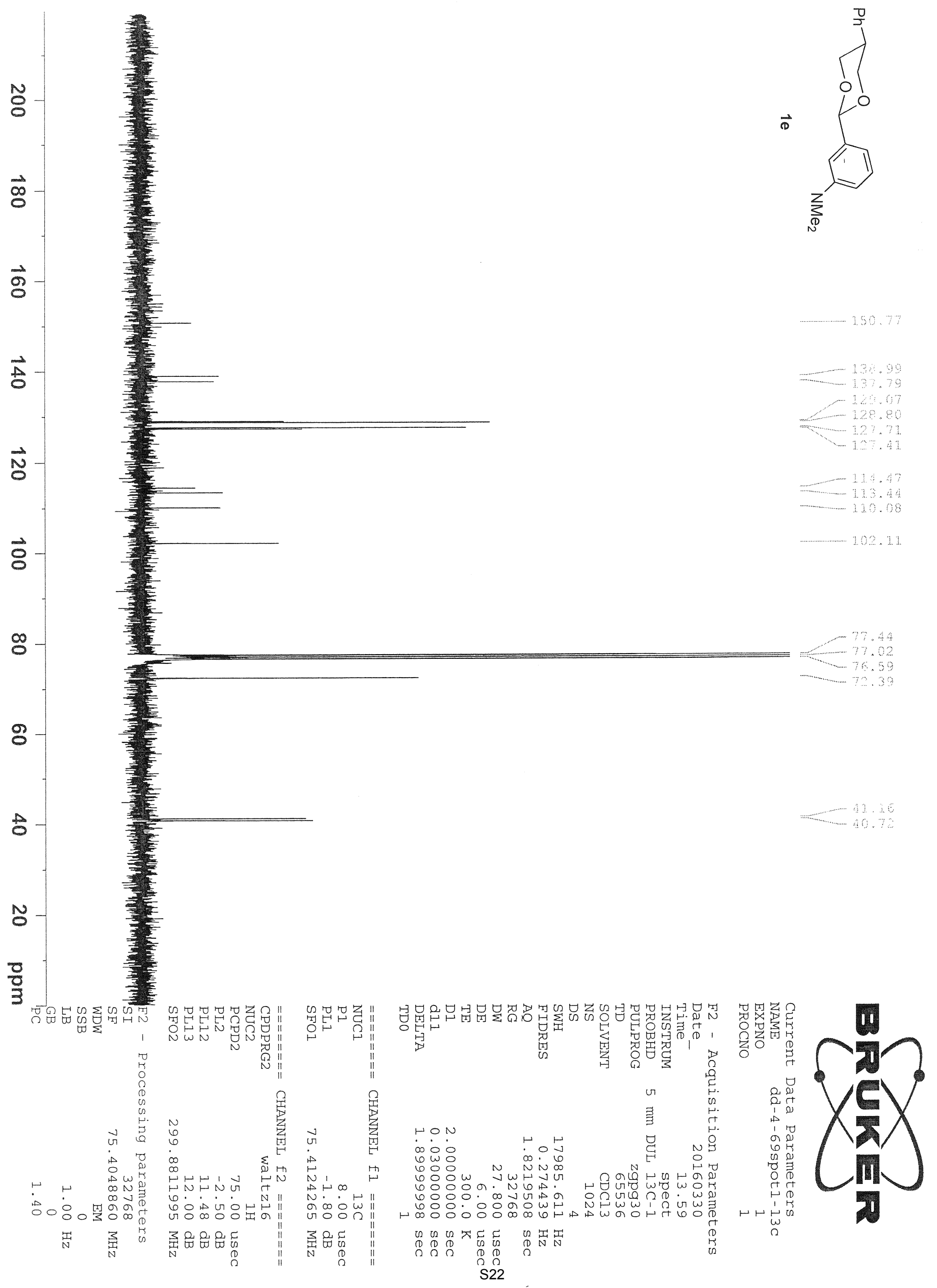



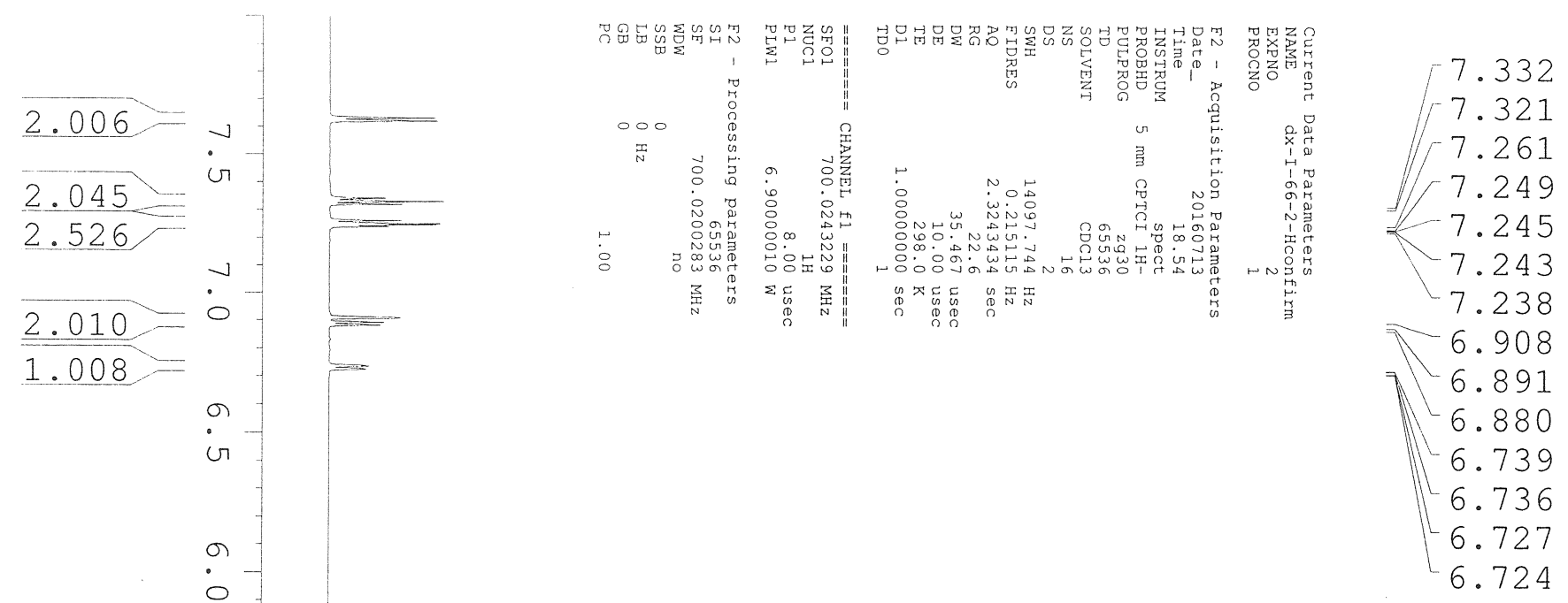

$1.000=G$
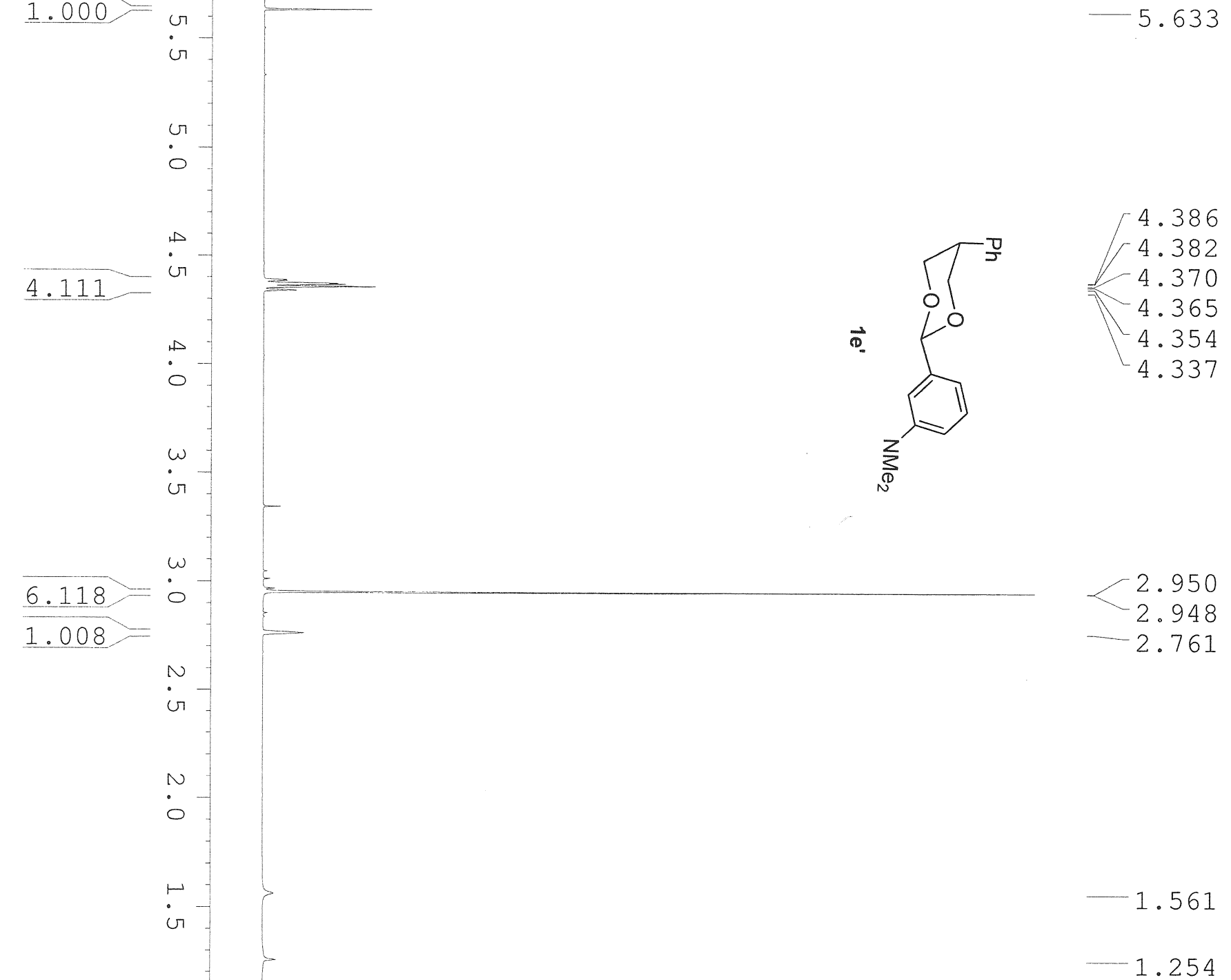


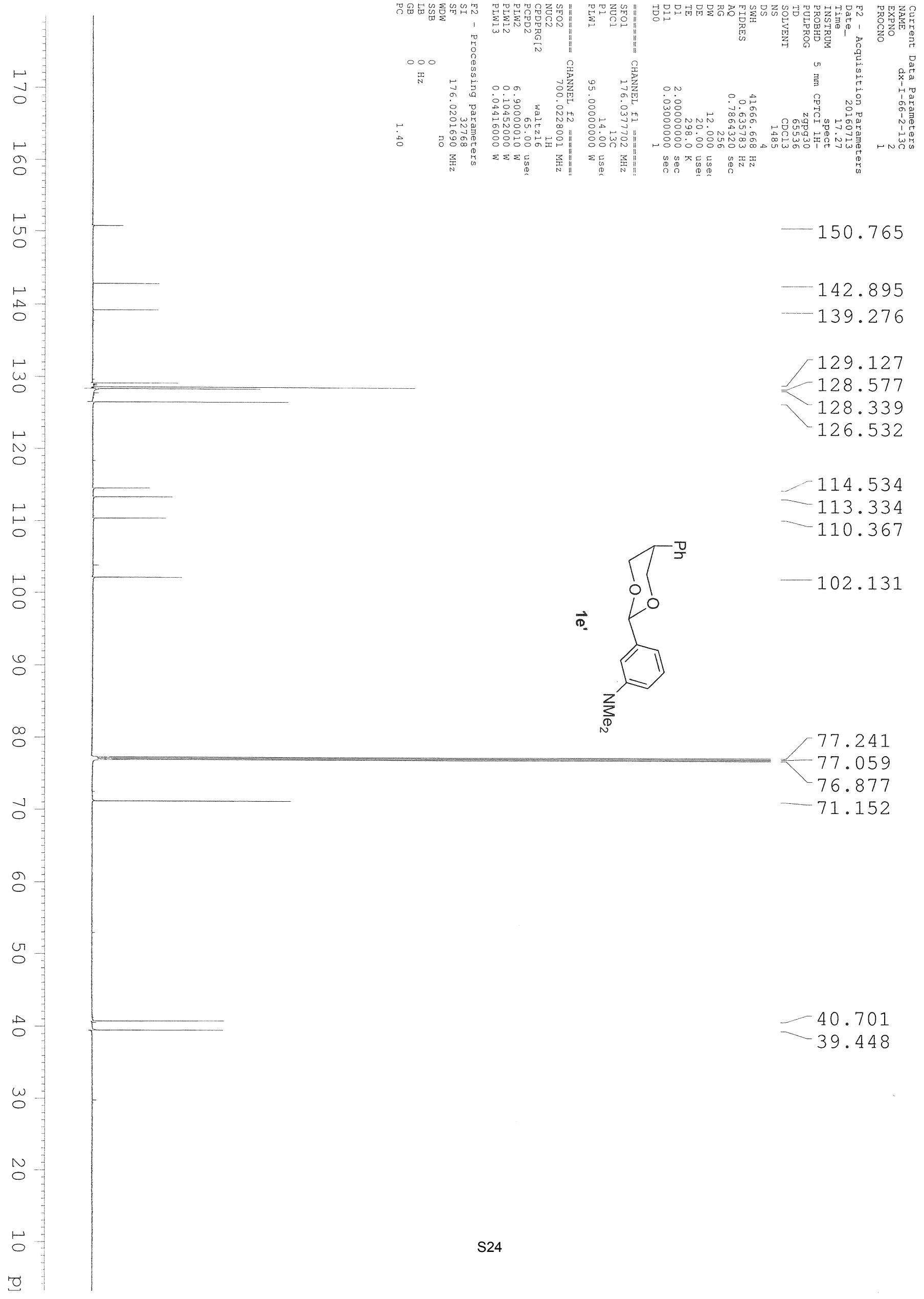



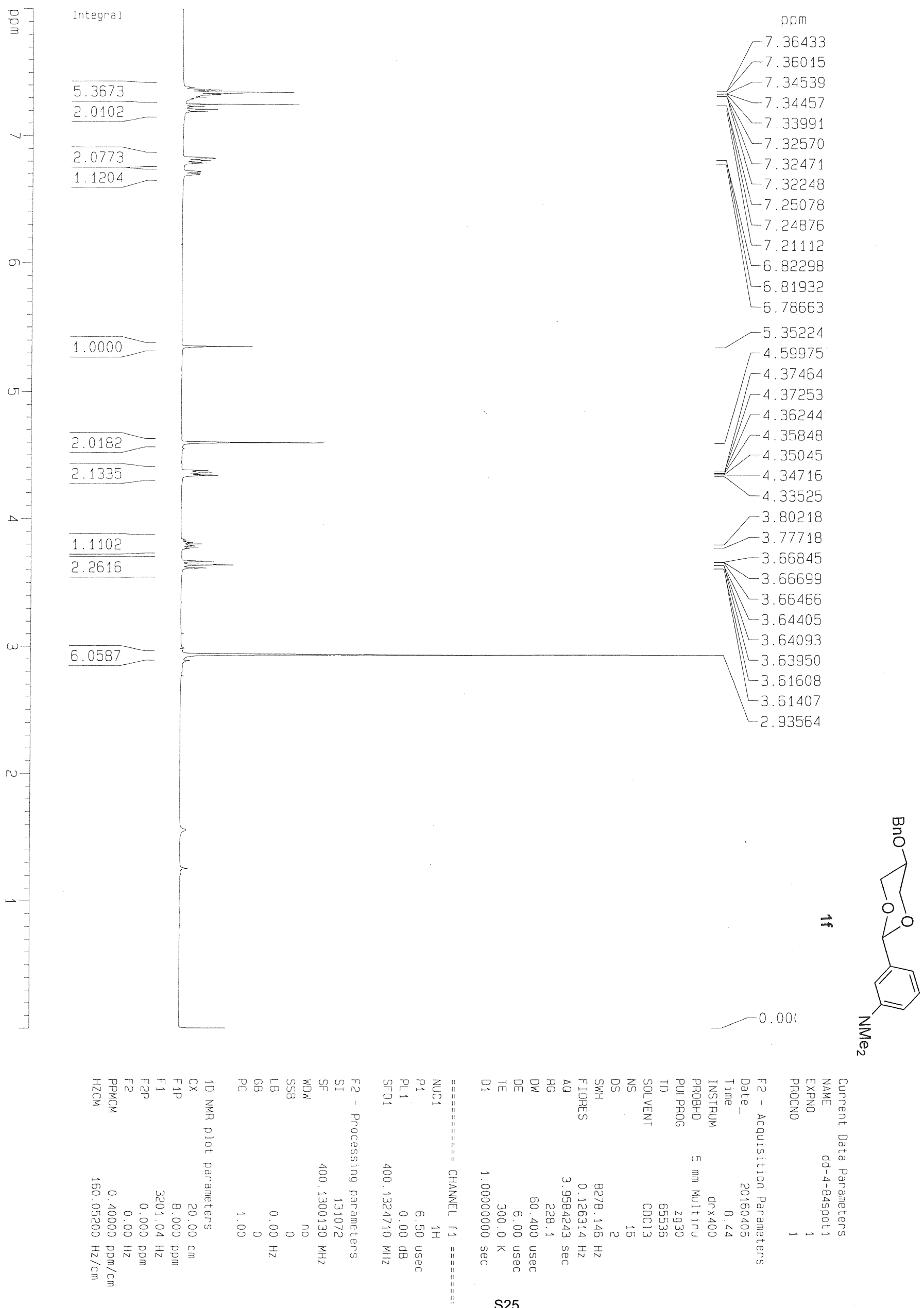


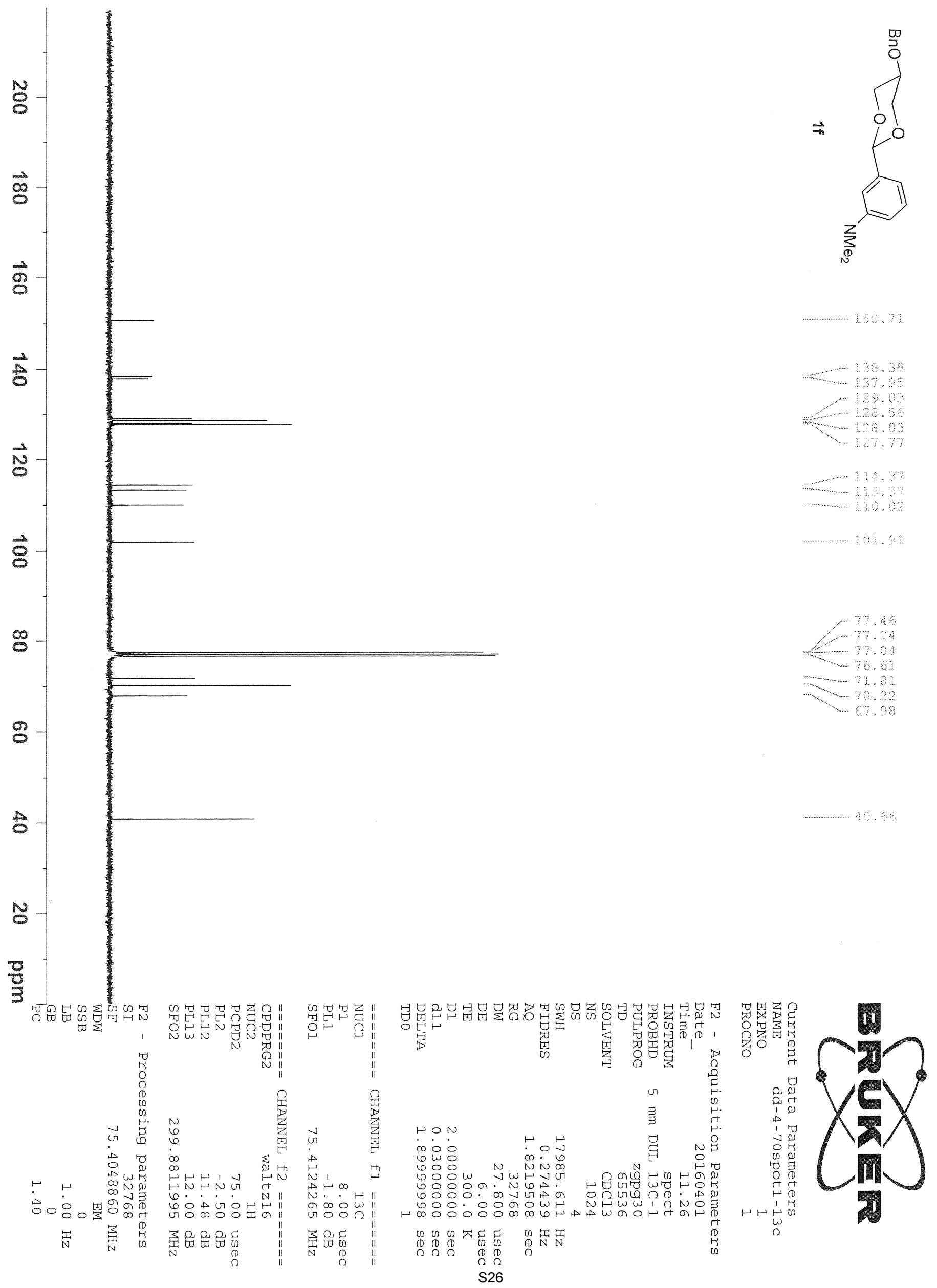



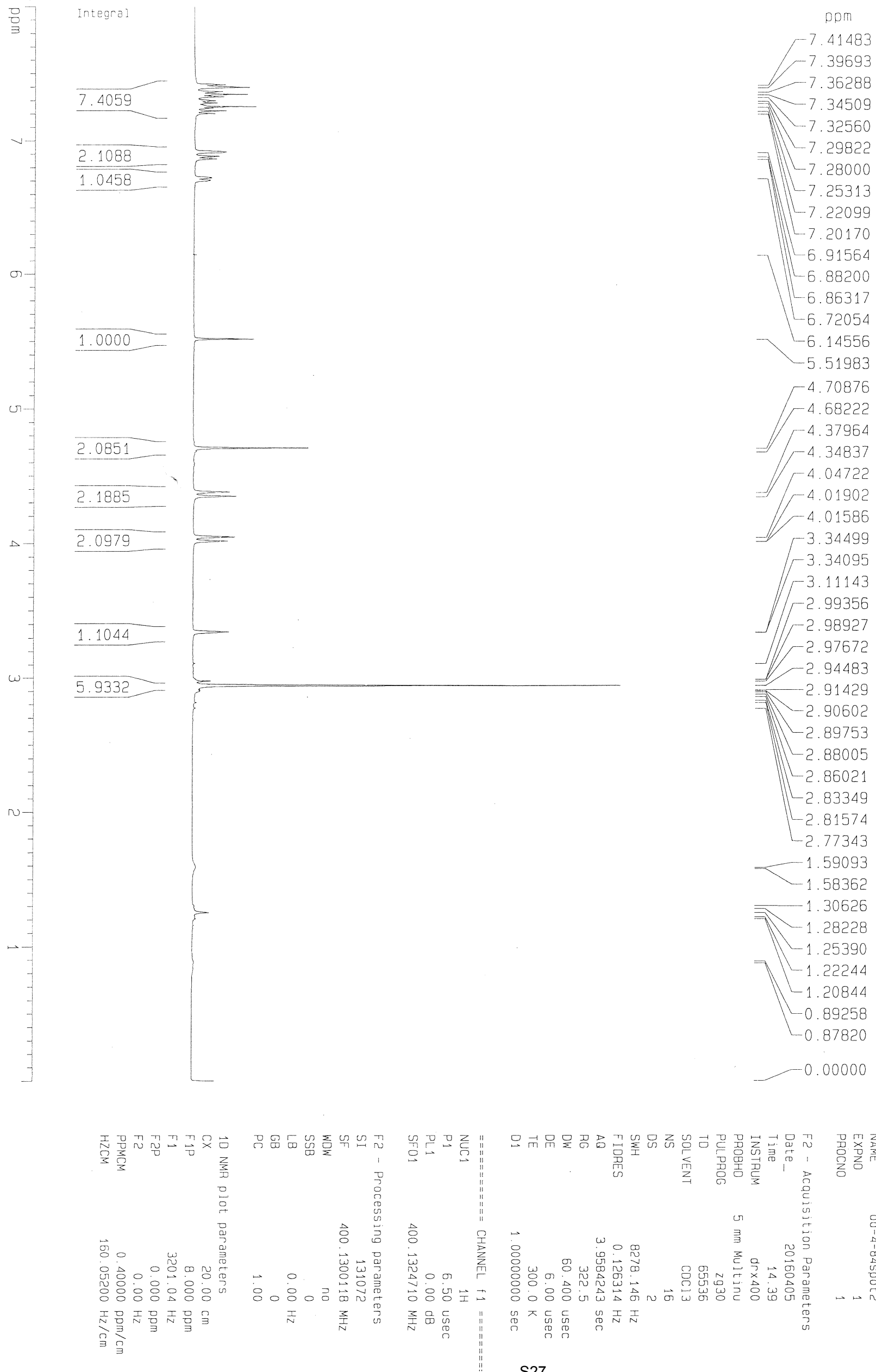

음 


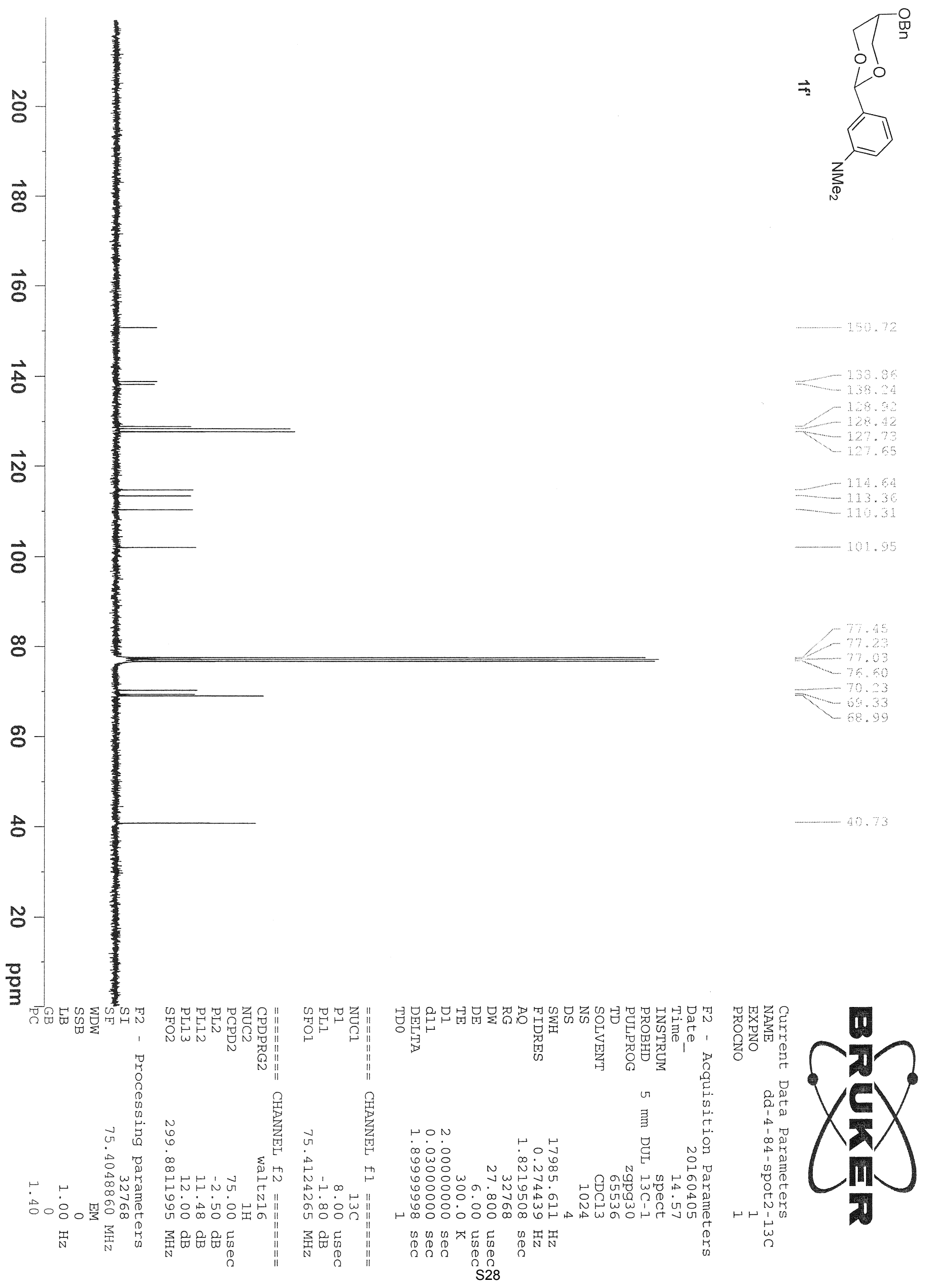




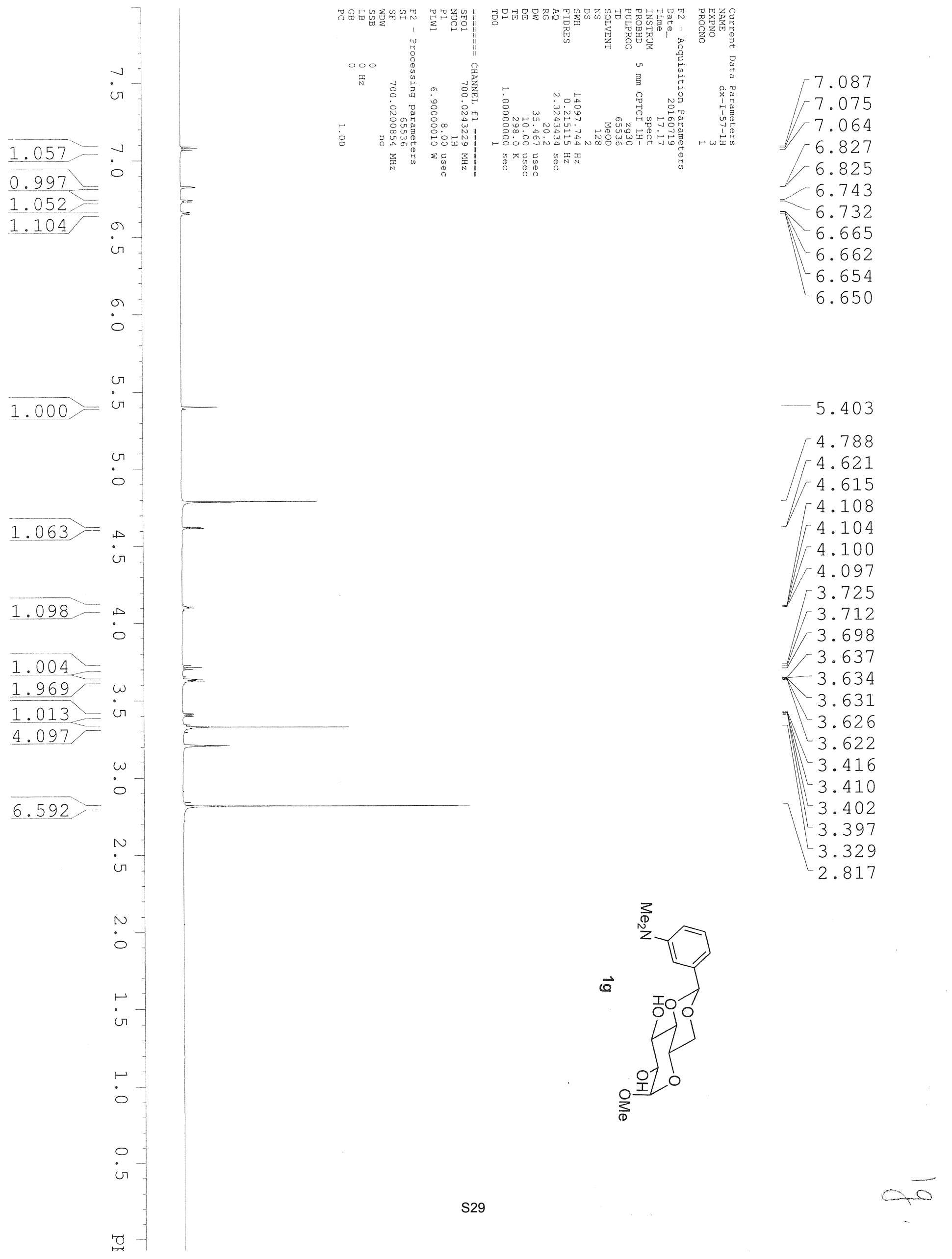




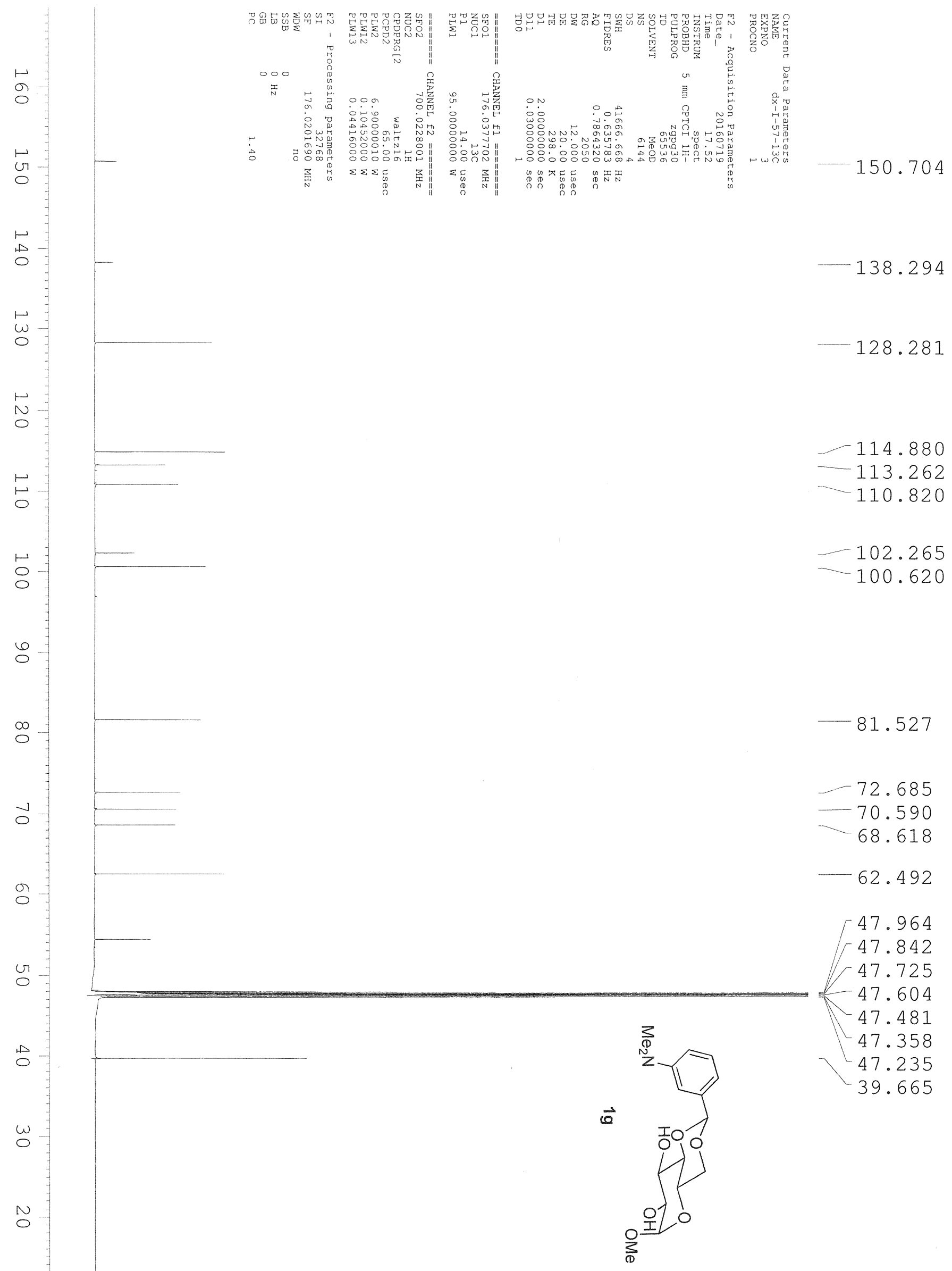



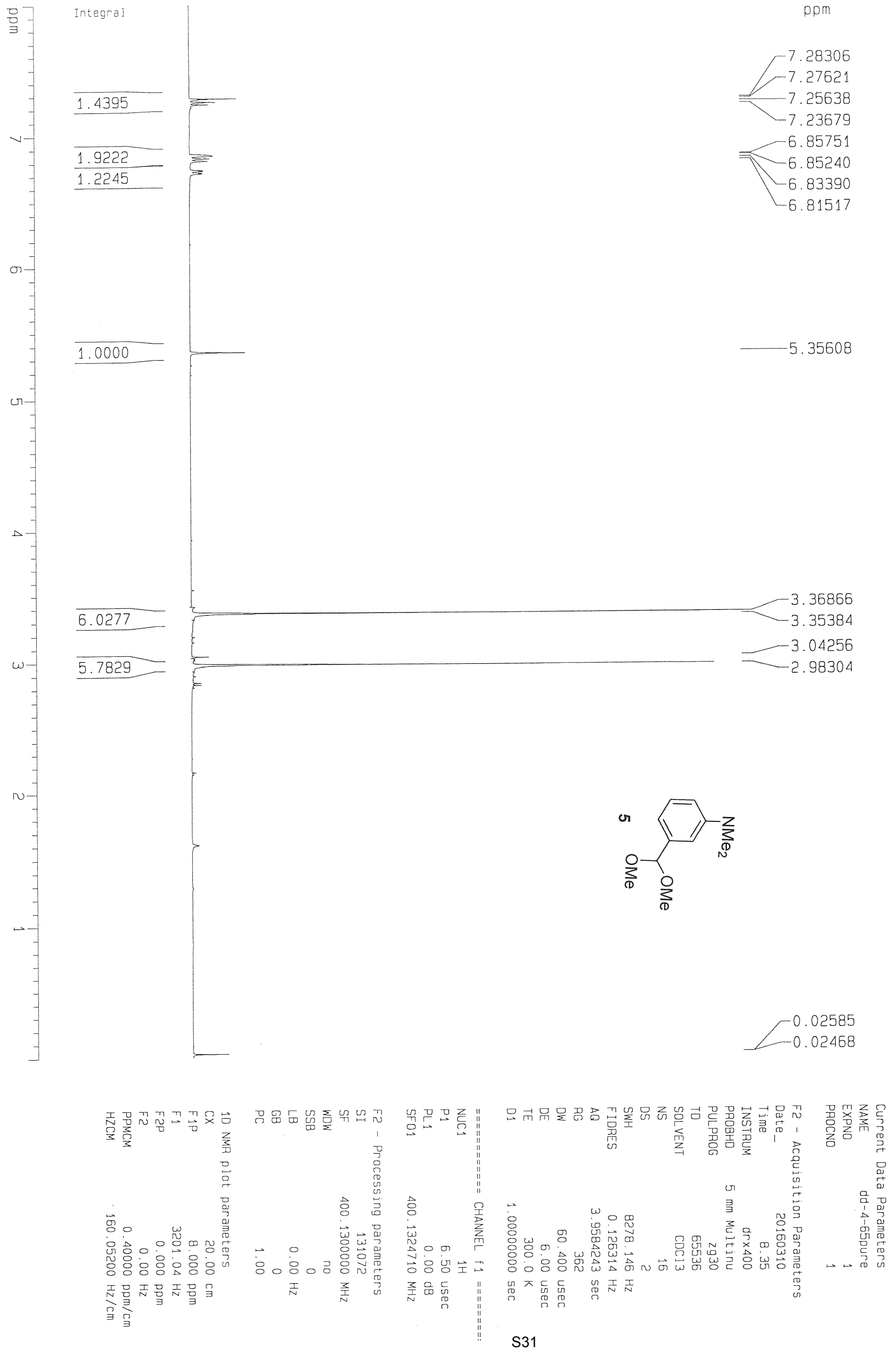


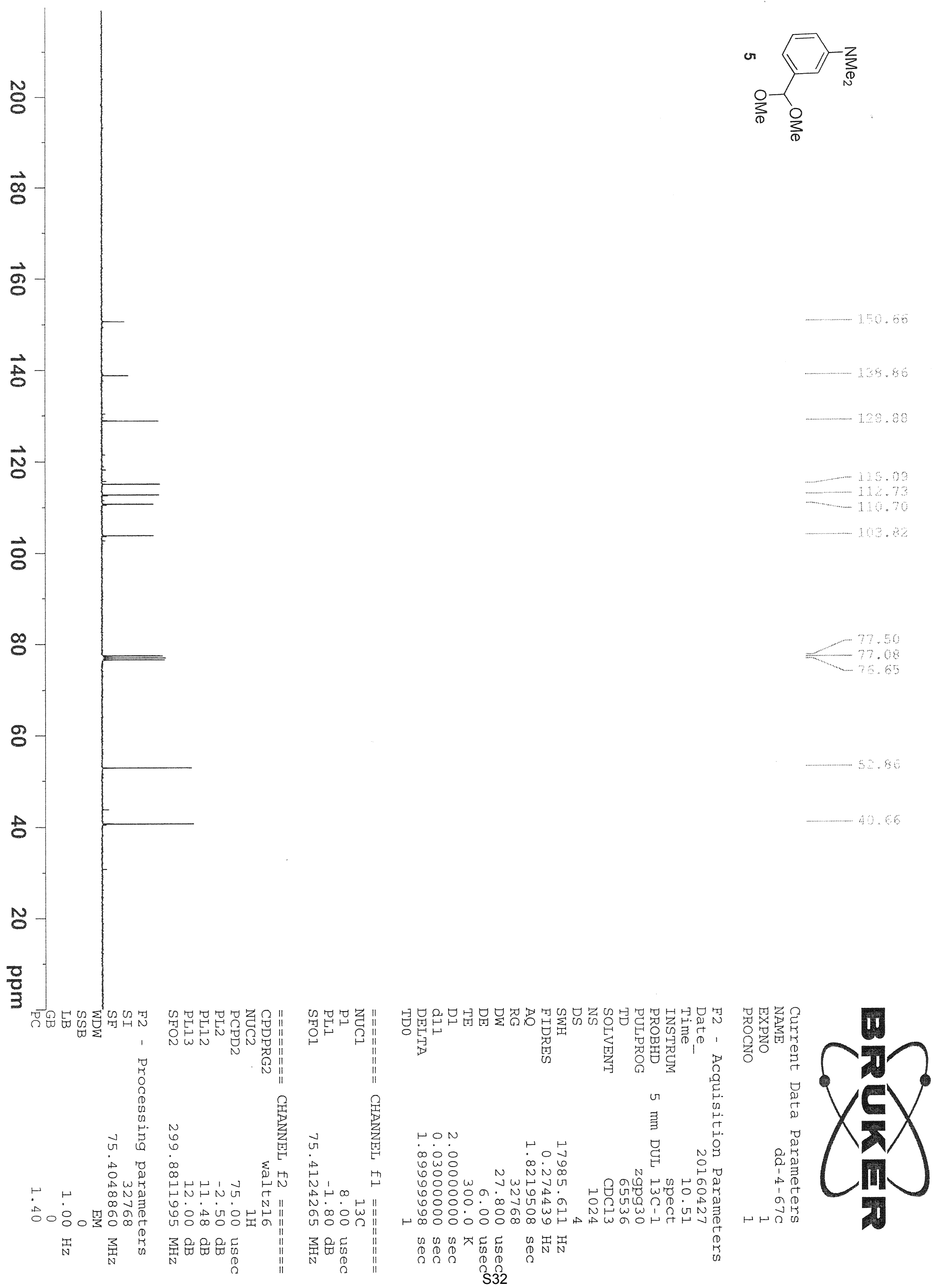




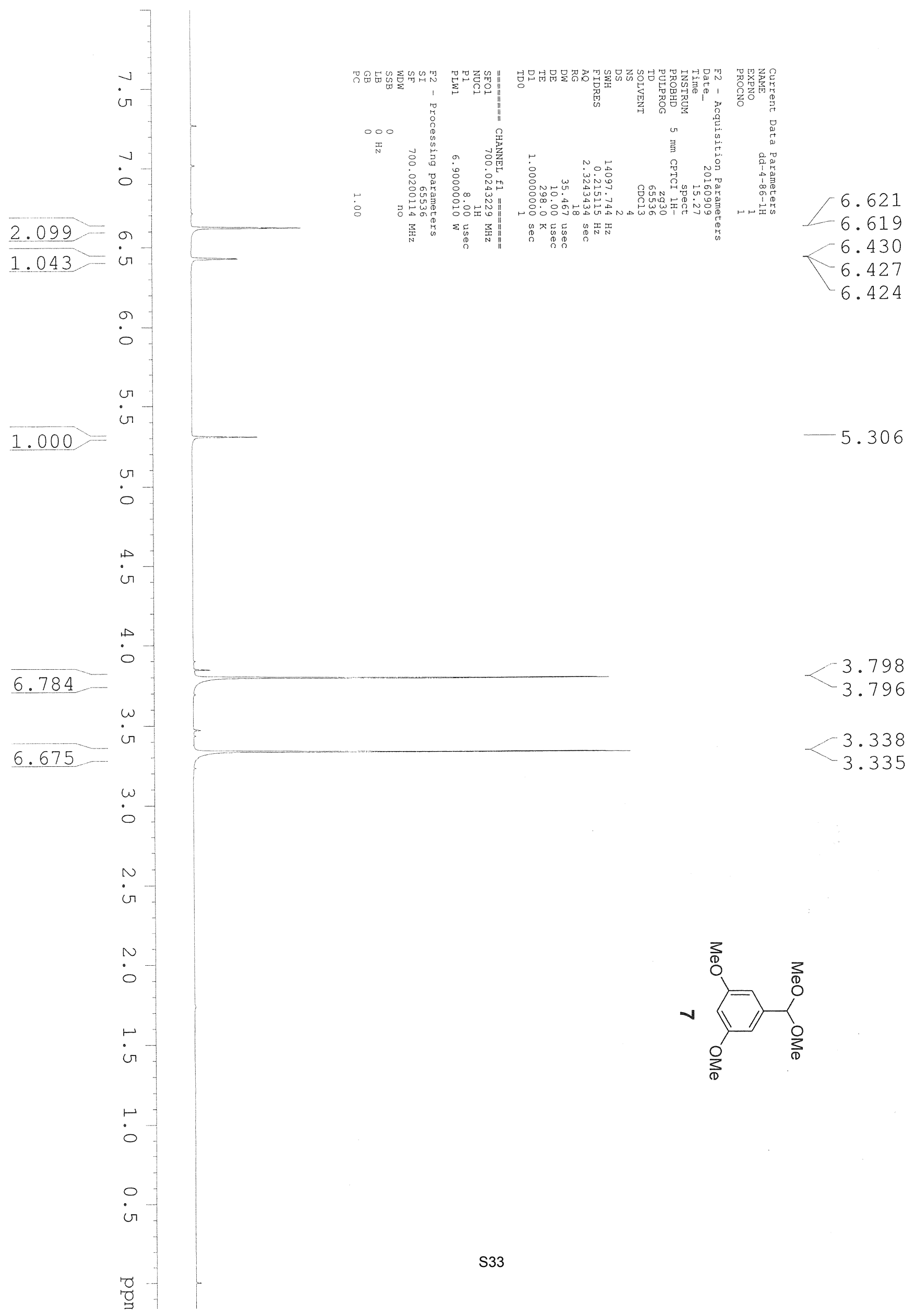




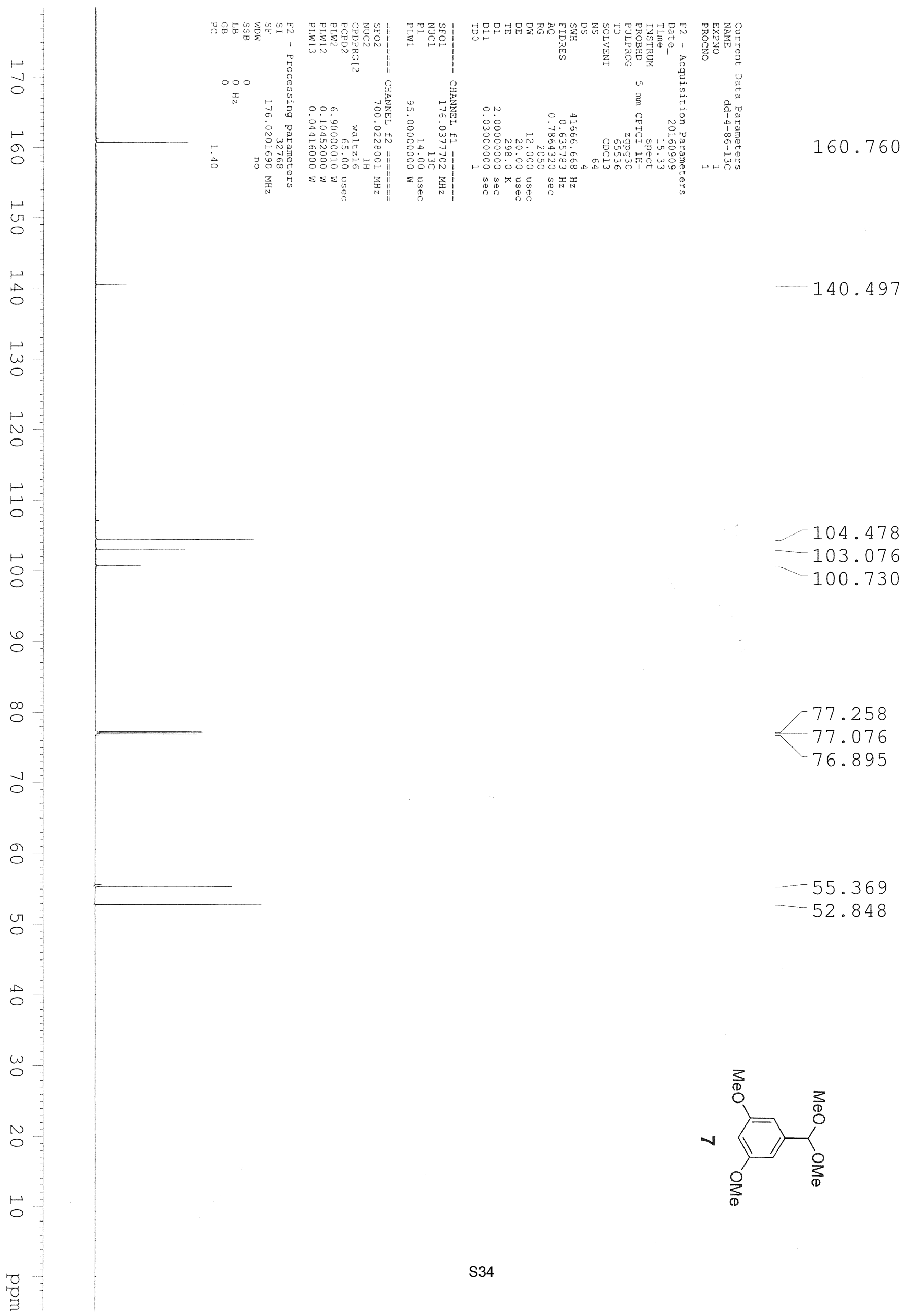




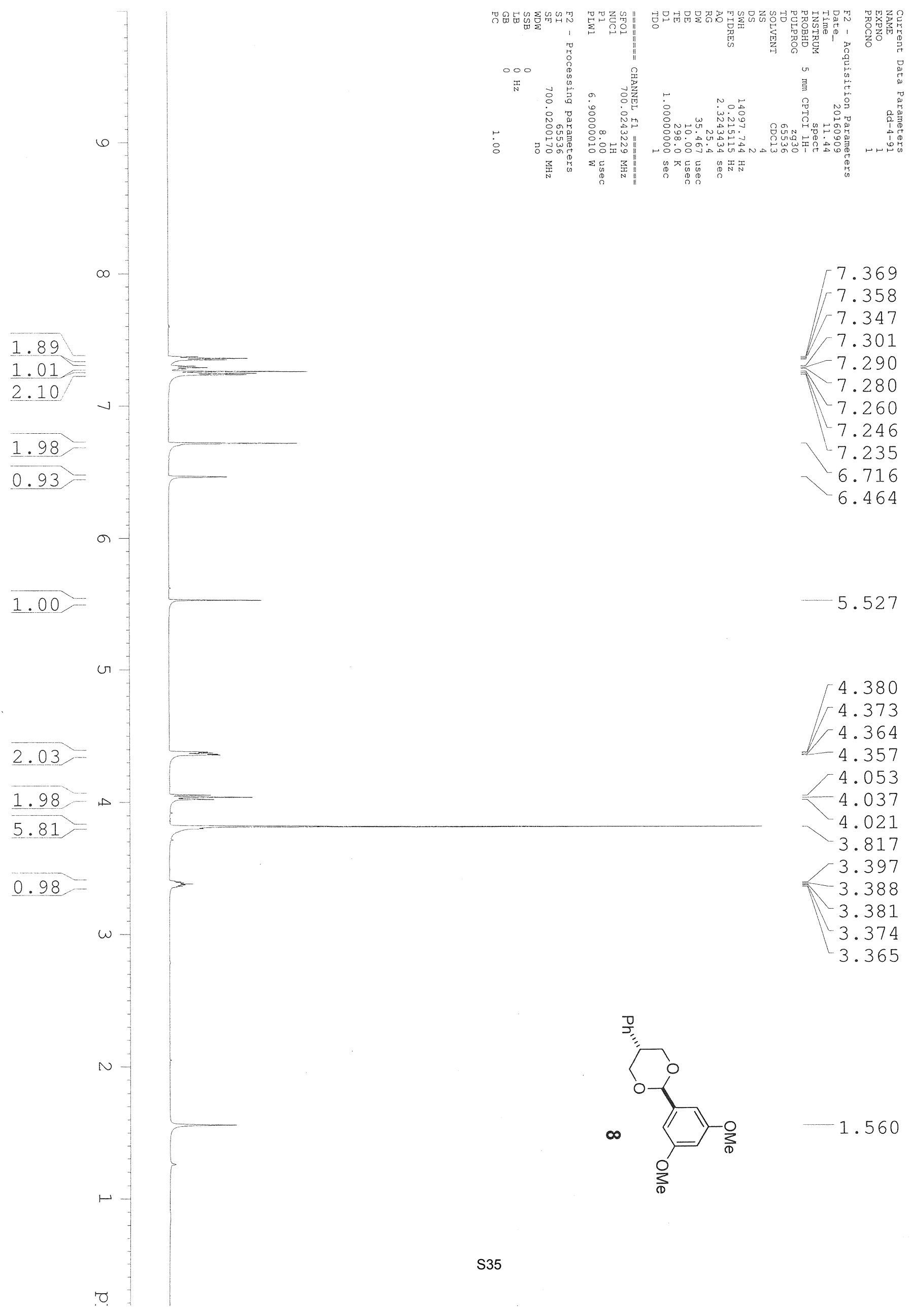




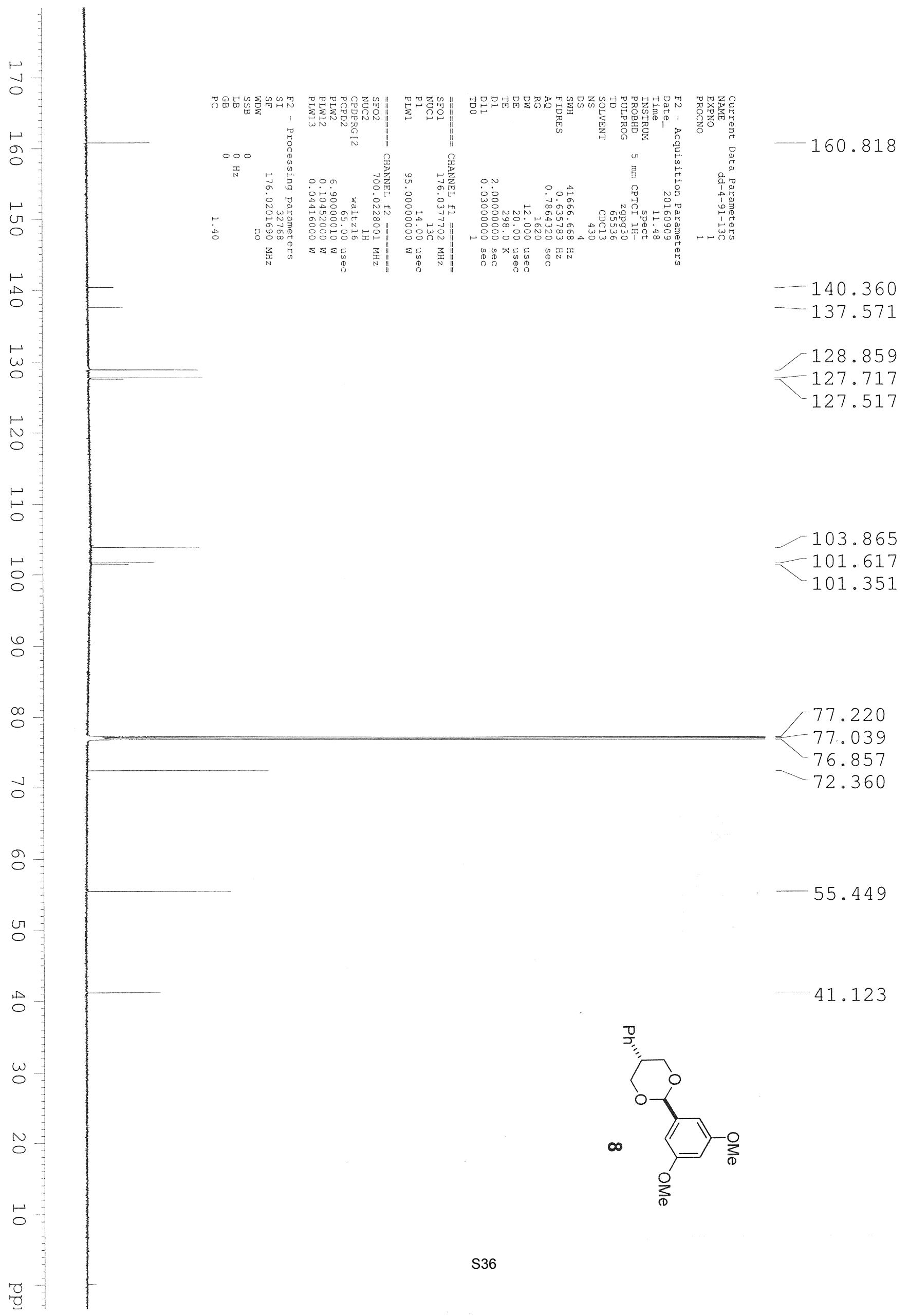



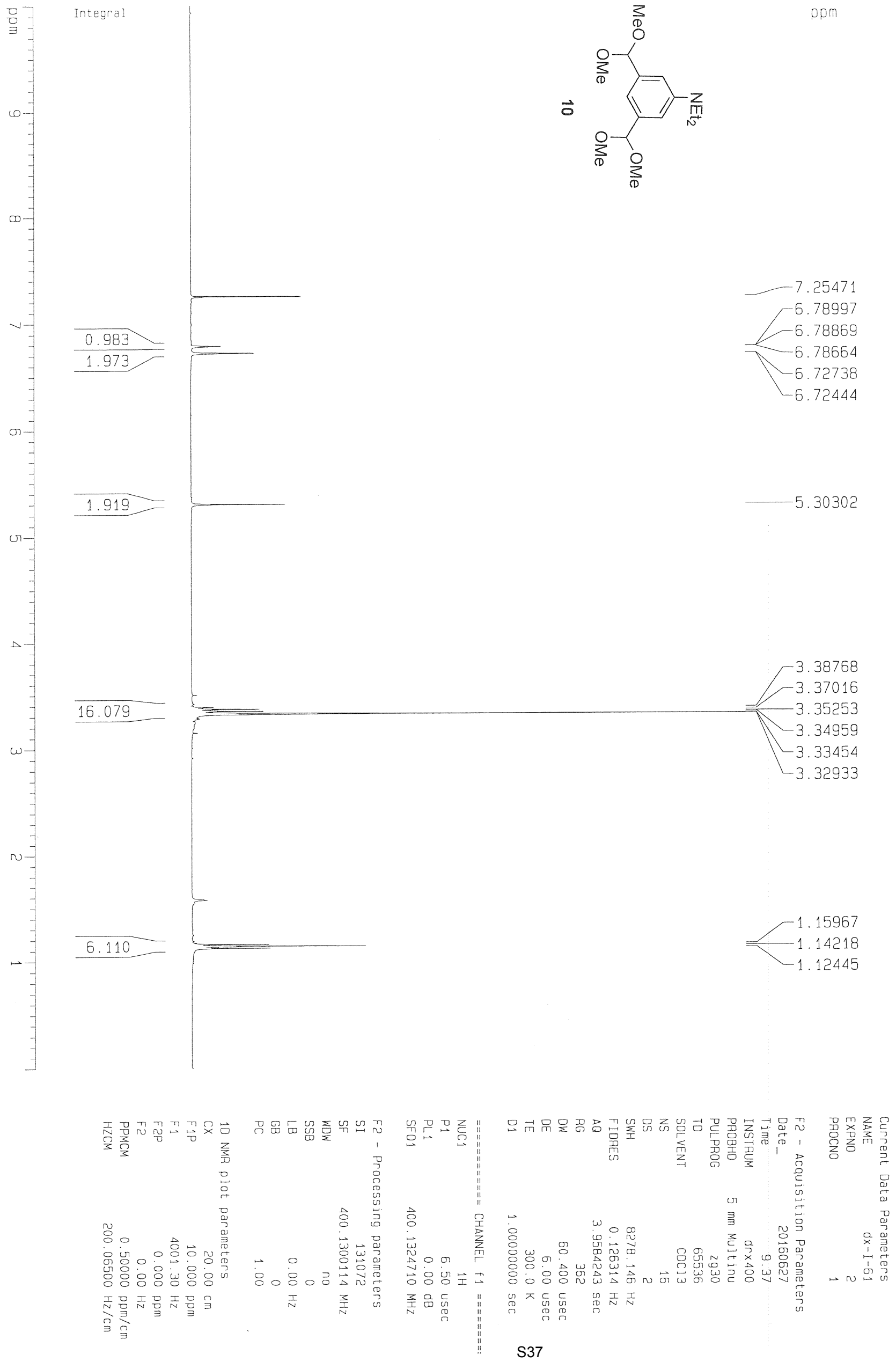


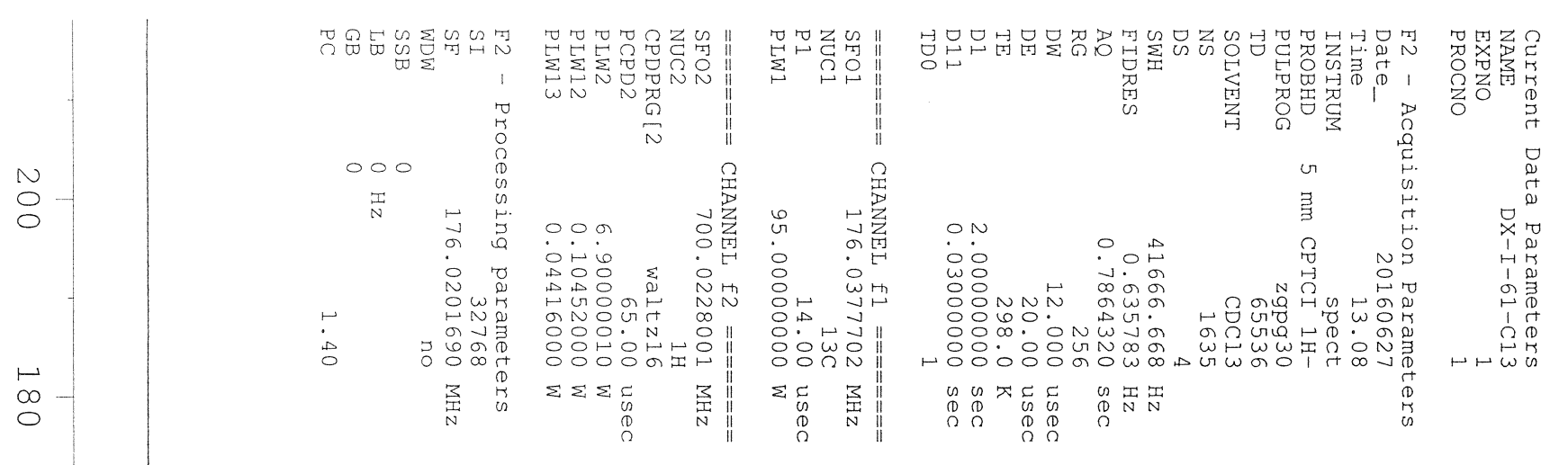

$\stackrel{\bullet}{\circ}$

$\stackrel{\bullet}{\circ}$

$\stackrel{\leftrightarrow}{\circ}$

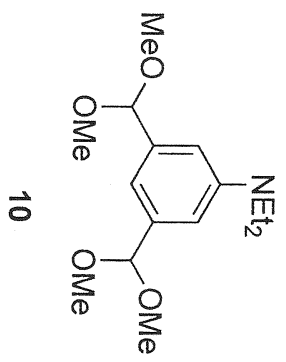

$-112.601$

138.911

$\stackrel{\bullet}{0}$

$\stackrel{\bullet}{\circ}$

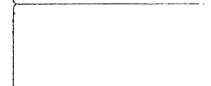

77.202

77.021

76.839

0

$-52.887$

$-44.325$

$\$$

$-12.585$

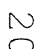




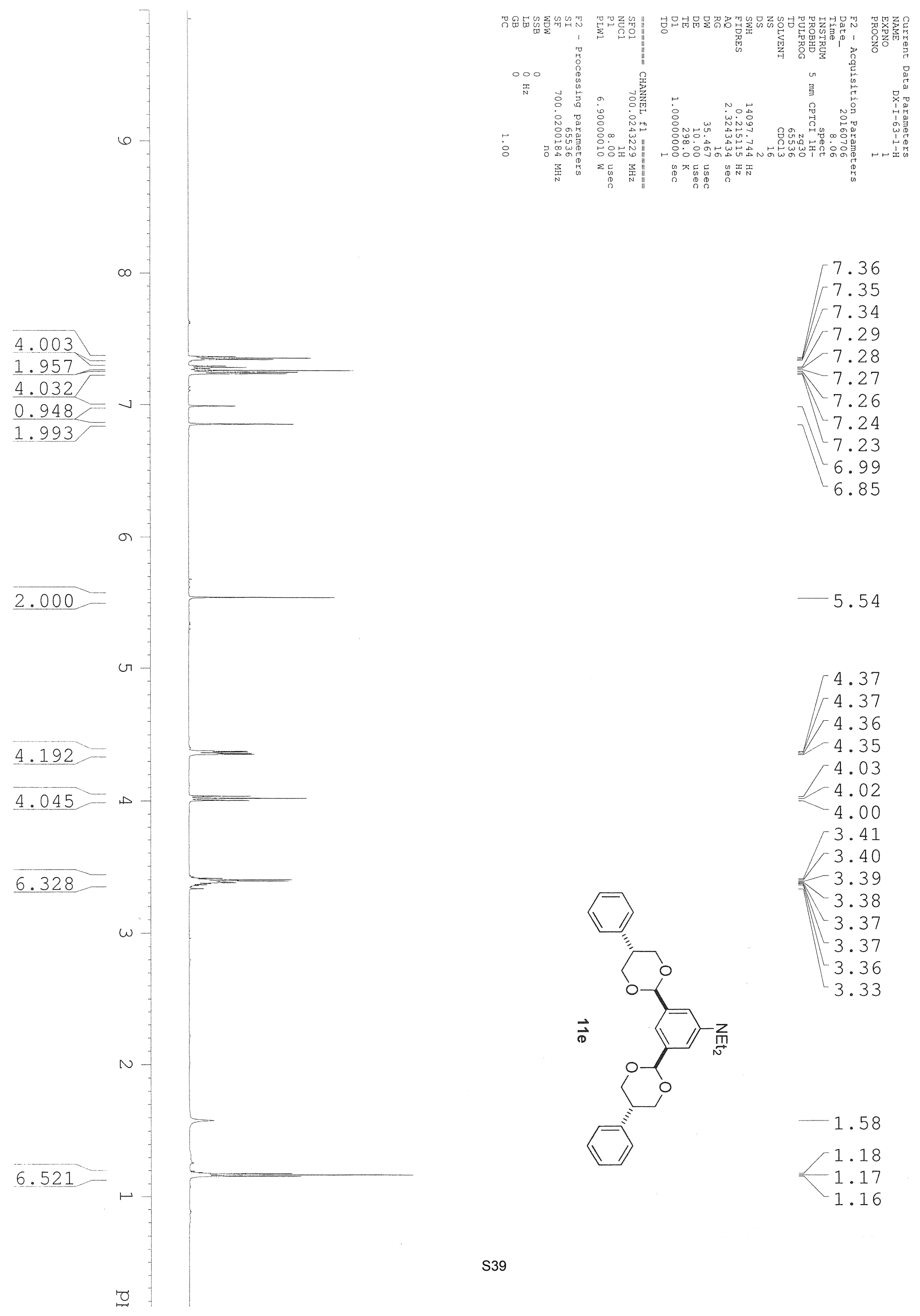




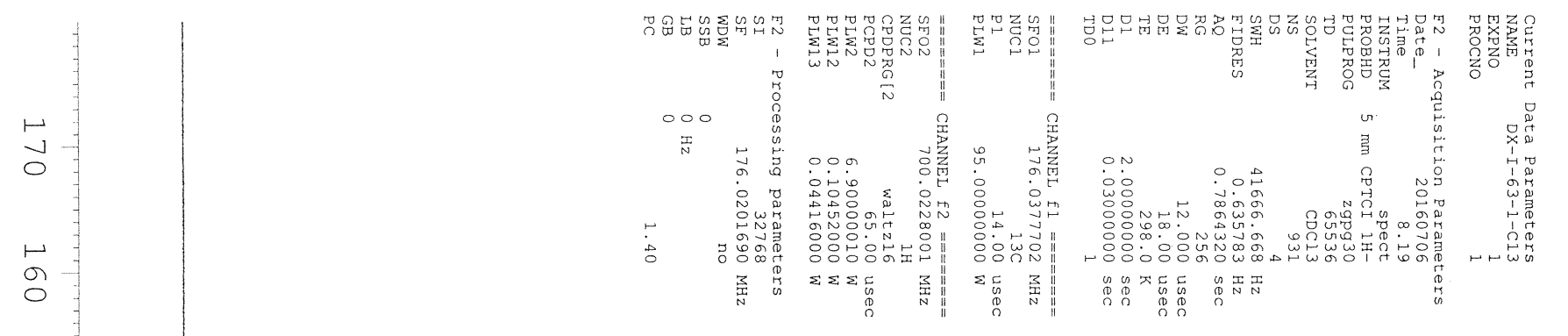

$-148.086$

$-139.272$

$-137.878$

$-128.769$

$-127.714$

$-111.199$

$-109.958$

$-101.911$

8

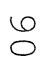

$\infty$

$\infty$

77.195

$-77.013$

$-76.830$

$-72.327$

๖े

$+$

๑

v

G

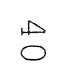
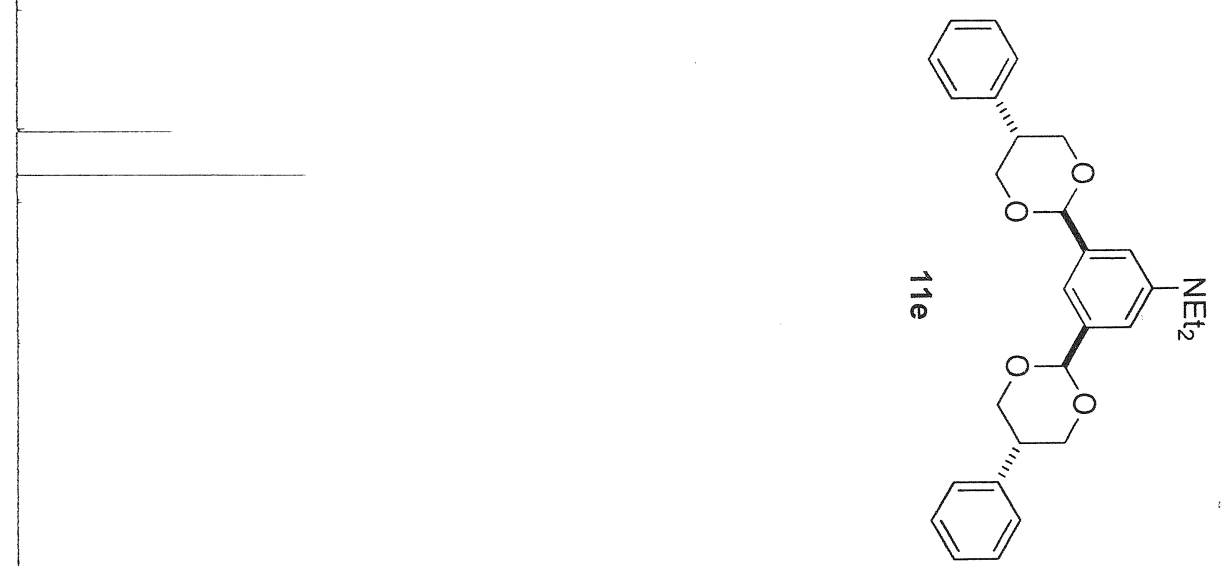

44.241

41.142

$w$

N

○

官 


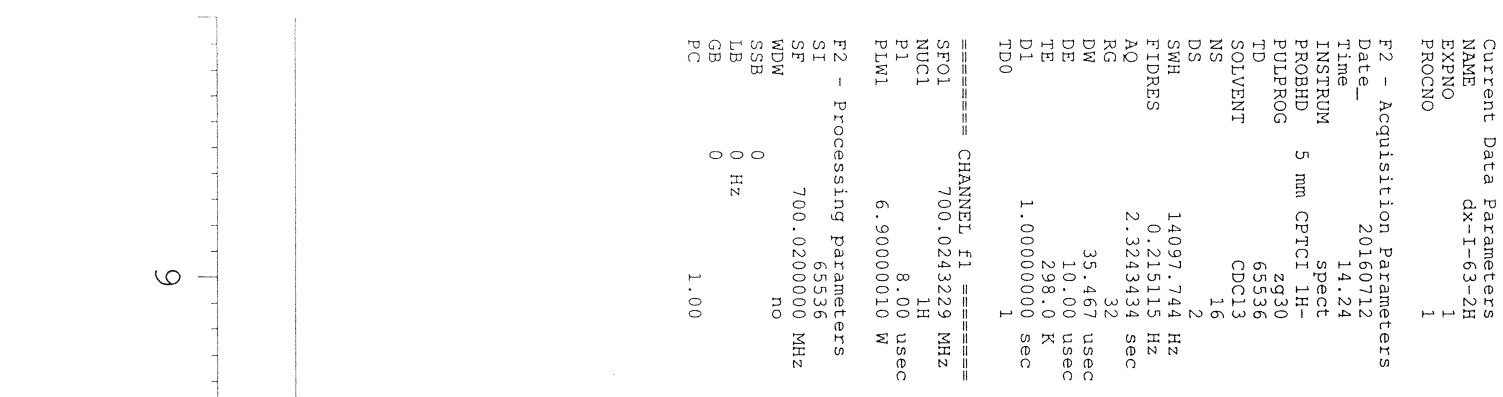

7.64

$-7.63$

$-7.38$

$-7.38$

$-7.37$

$-7.37$

$-7.36$

$-7.36$

7.35

7.34

7.34

7.31

$-7.31$

$-7.29$

7.28

7.27

$-7.26$

6.97

6.90

$-6.87$

5.68

5.56

4.39

4.39

4.38

4.37

$-4.37$

$-4.36$

4.36

4.36

4.34

$-4.06$

$-4.04$

4.03

$-3.43$

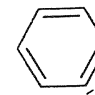

3.42

3. 41

3.40

2.80

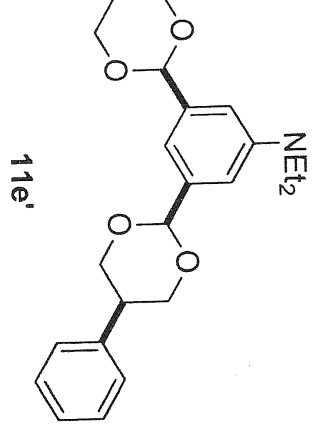

1.20

1.20

- 1.19

$-1.19$

1.18 

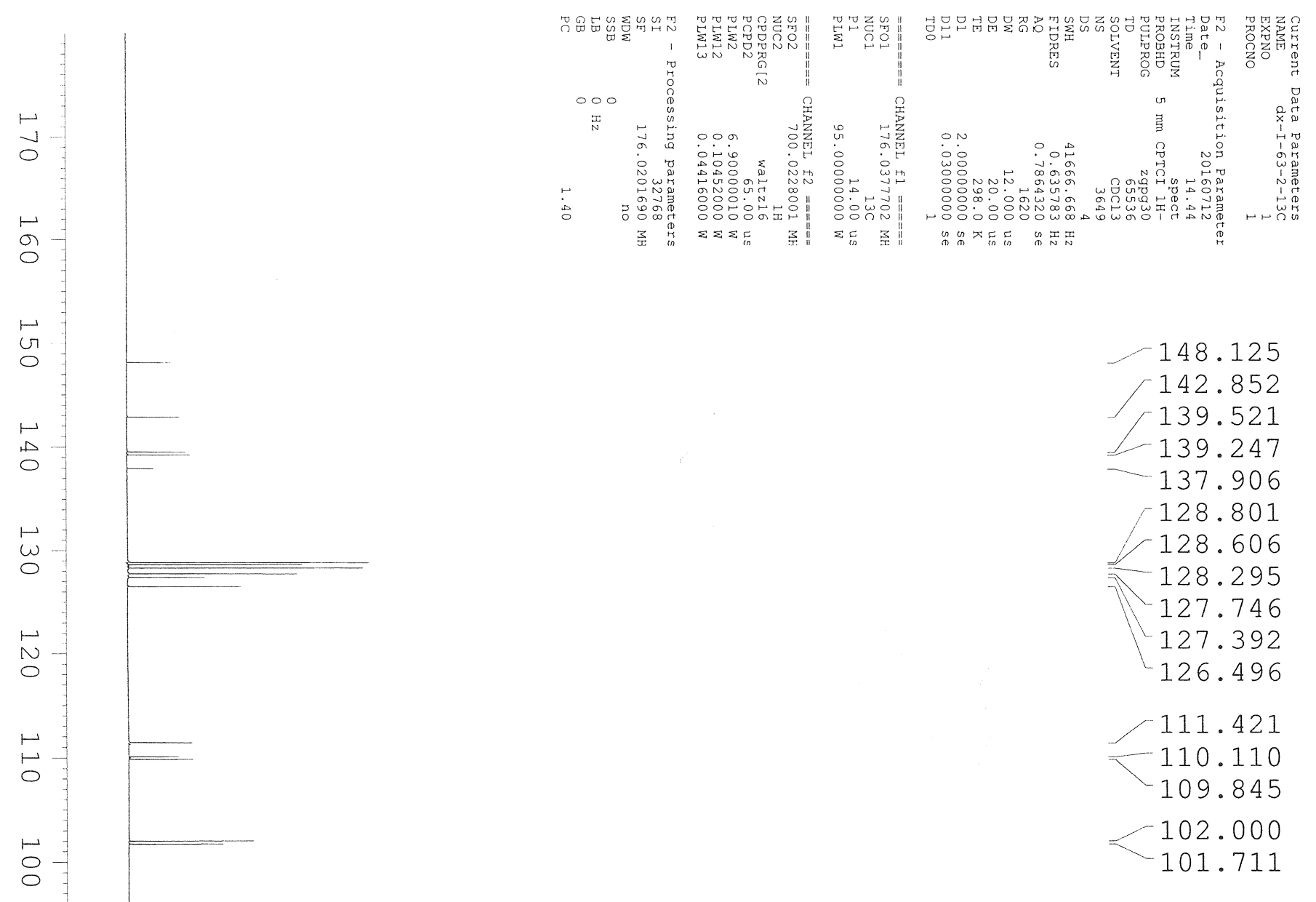

6

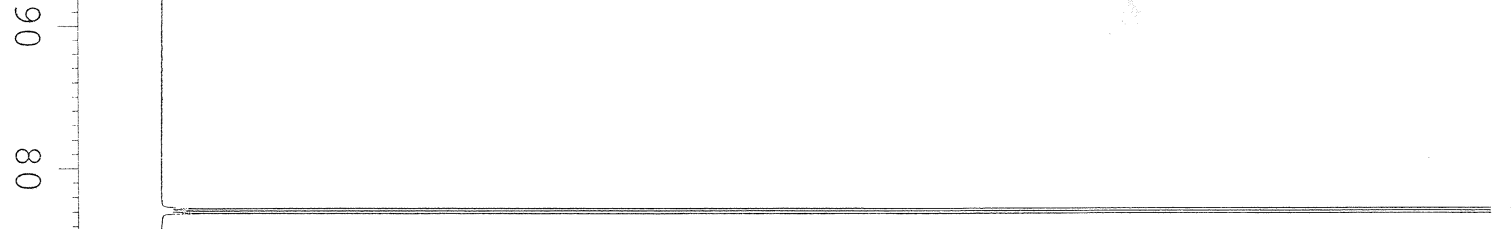

$-77.228$

77.046

76.864

72.360

70.879

J

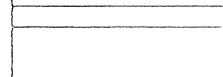

o
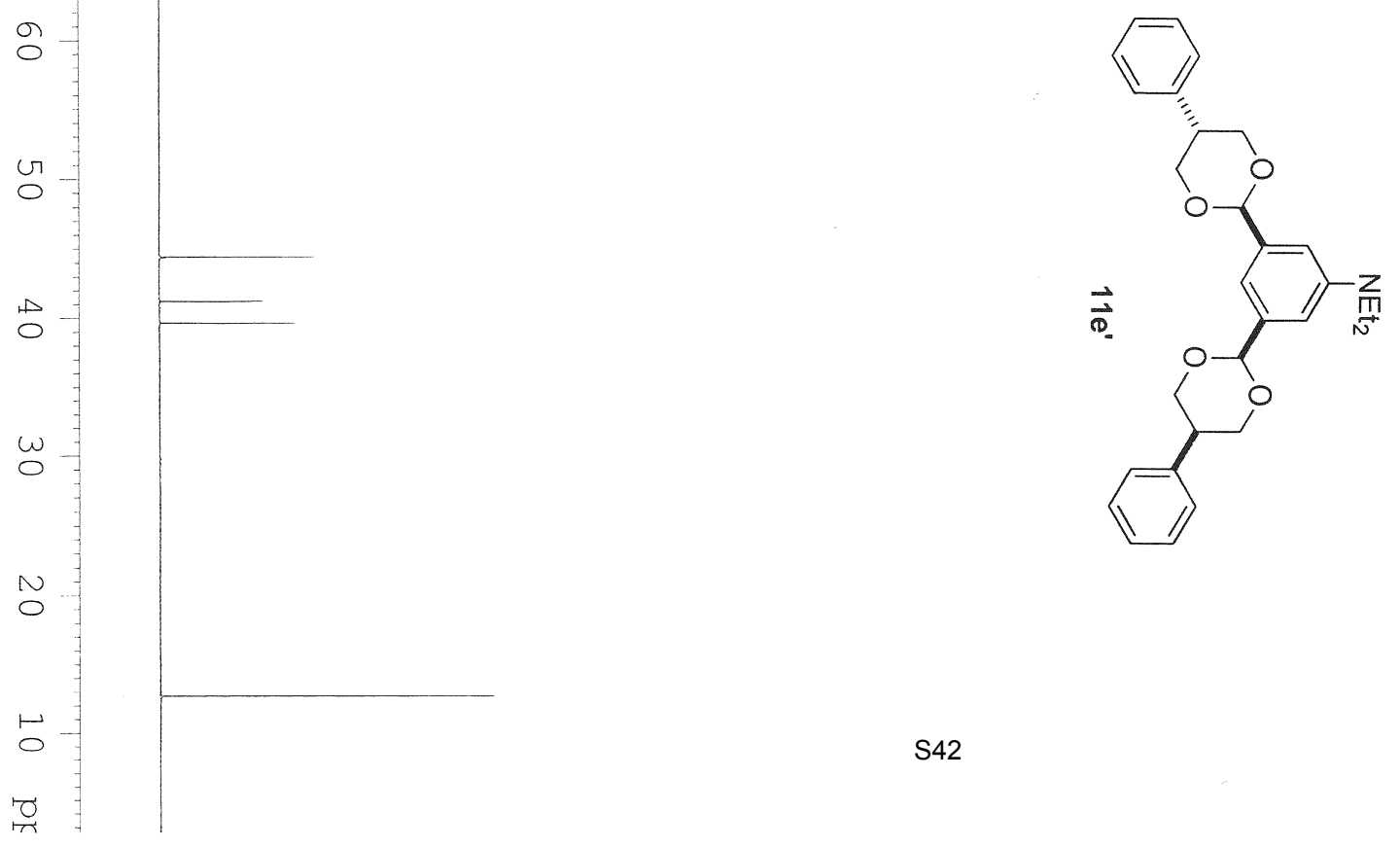

$-44.365$

$-41.173$

$-39.606$ 


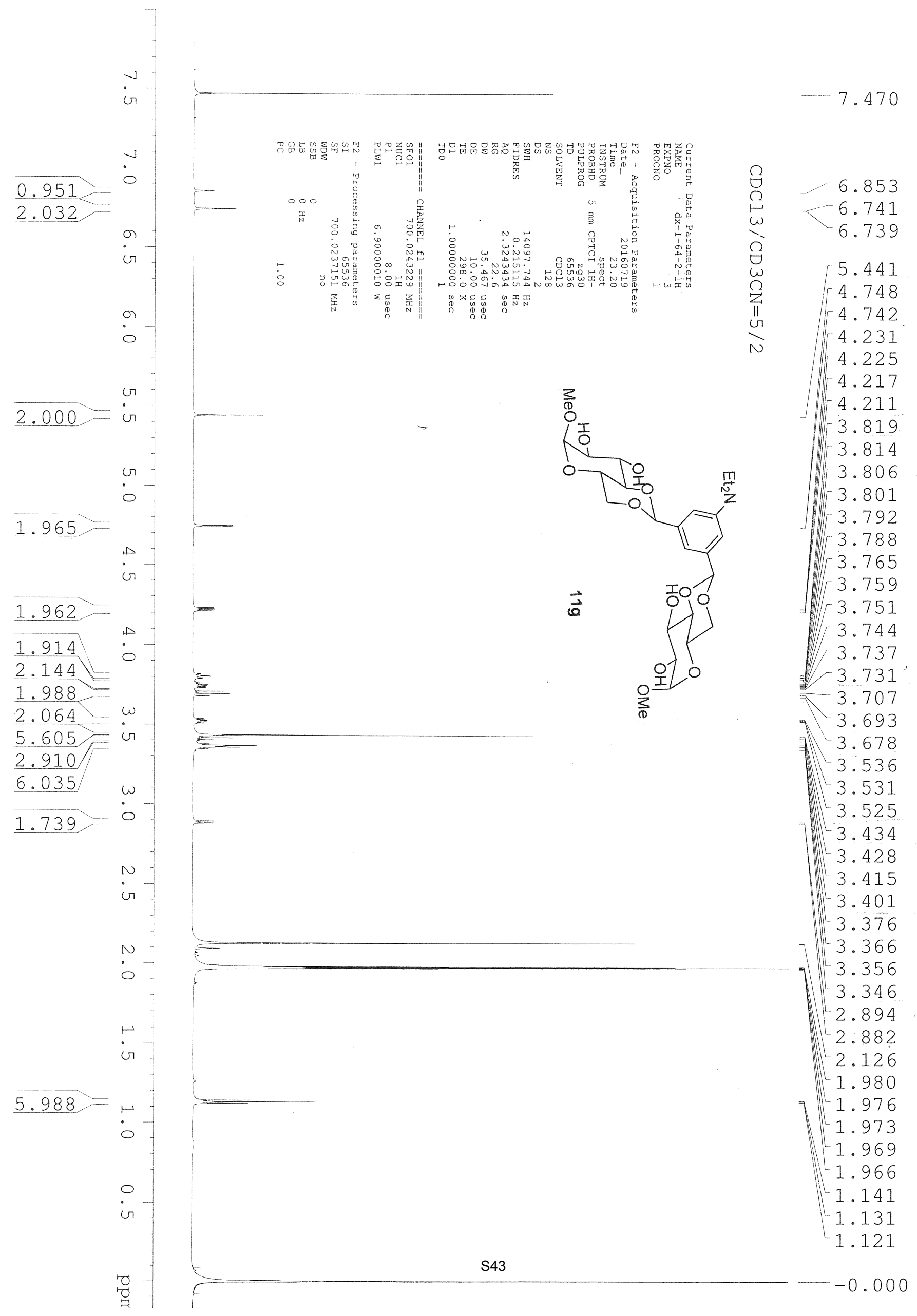



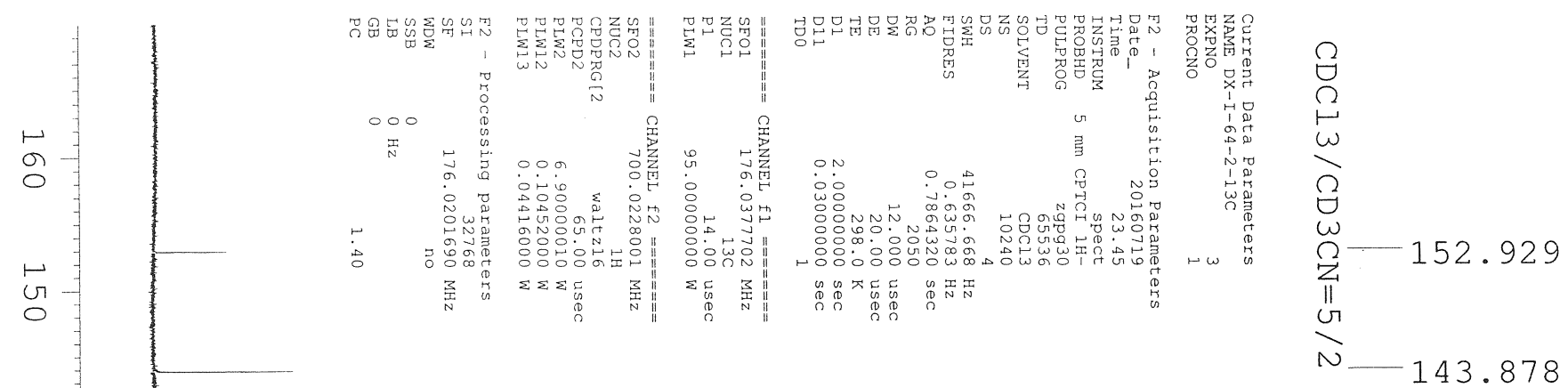

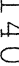

$\omega$

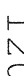

官

๖

6

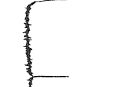

122.297

$-116.826$

115.789

$-107.537$

$-107.519$

$-105.468$

86.513

83.285

83.101

$\infty$

82.916

78.190

76.391

74.116

○े

3

o

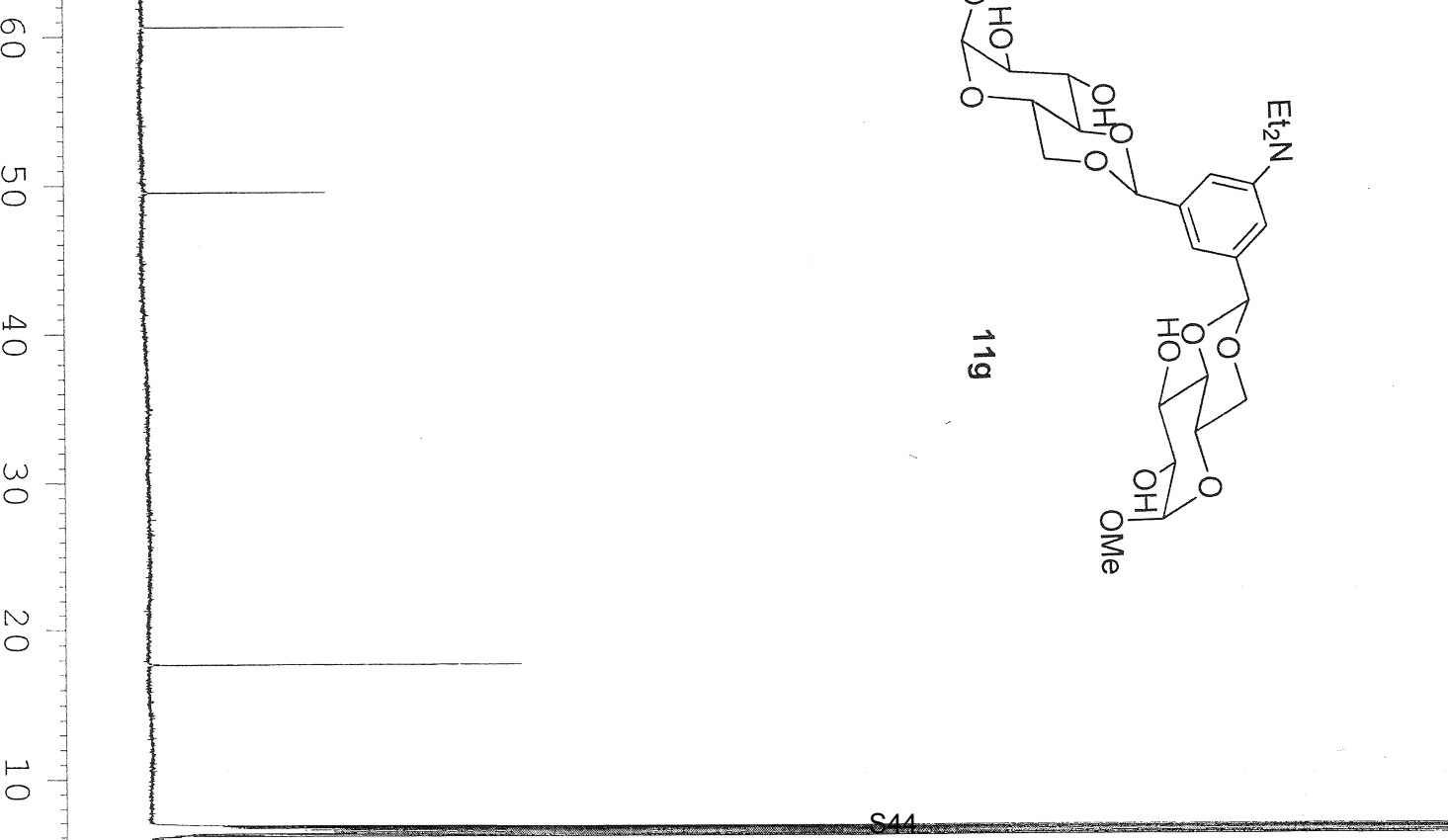

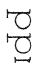

1

17.639
6.687
6.571
6.454
6.333
6.217




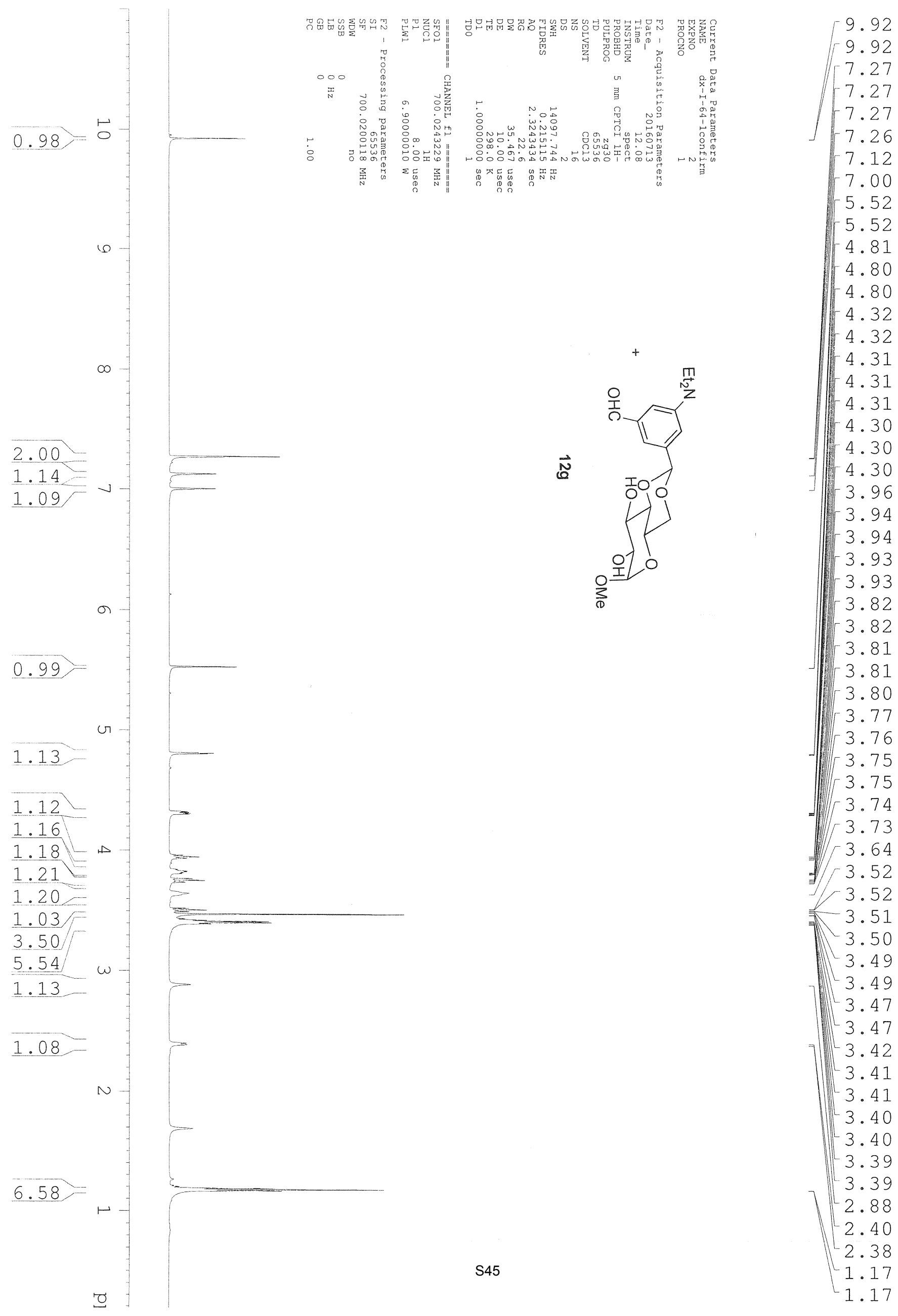




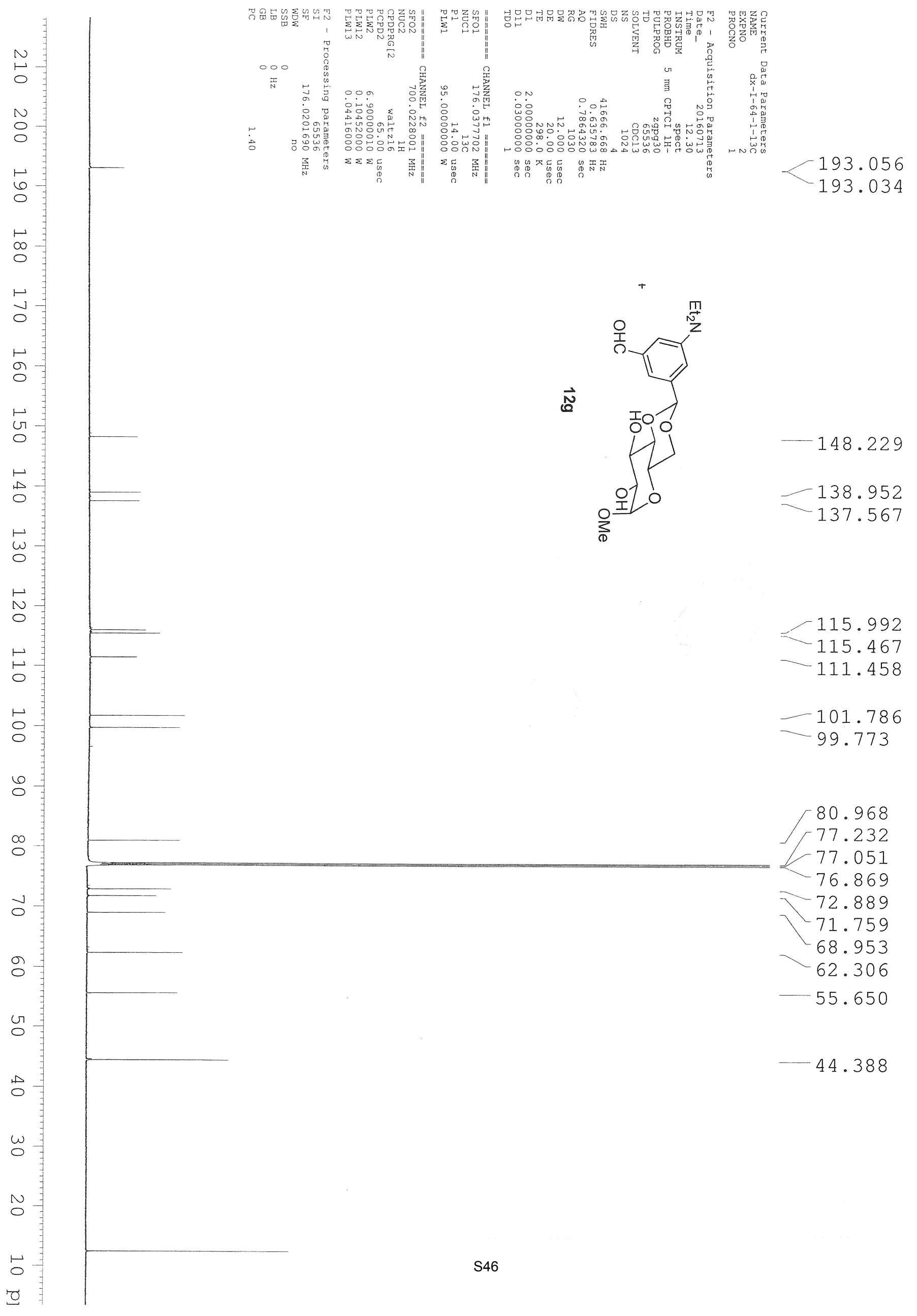



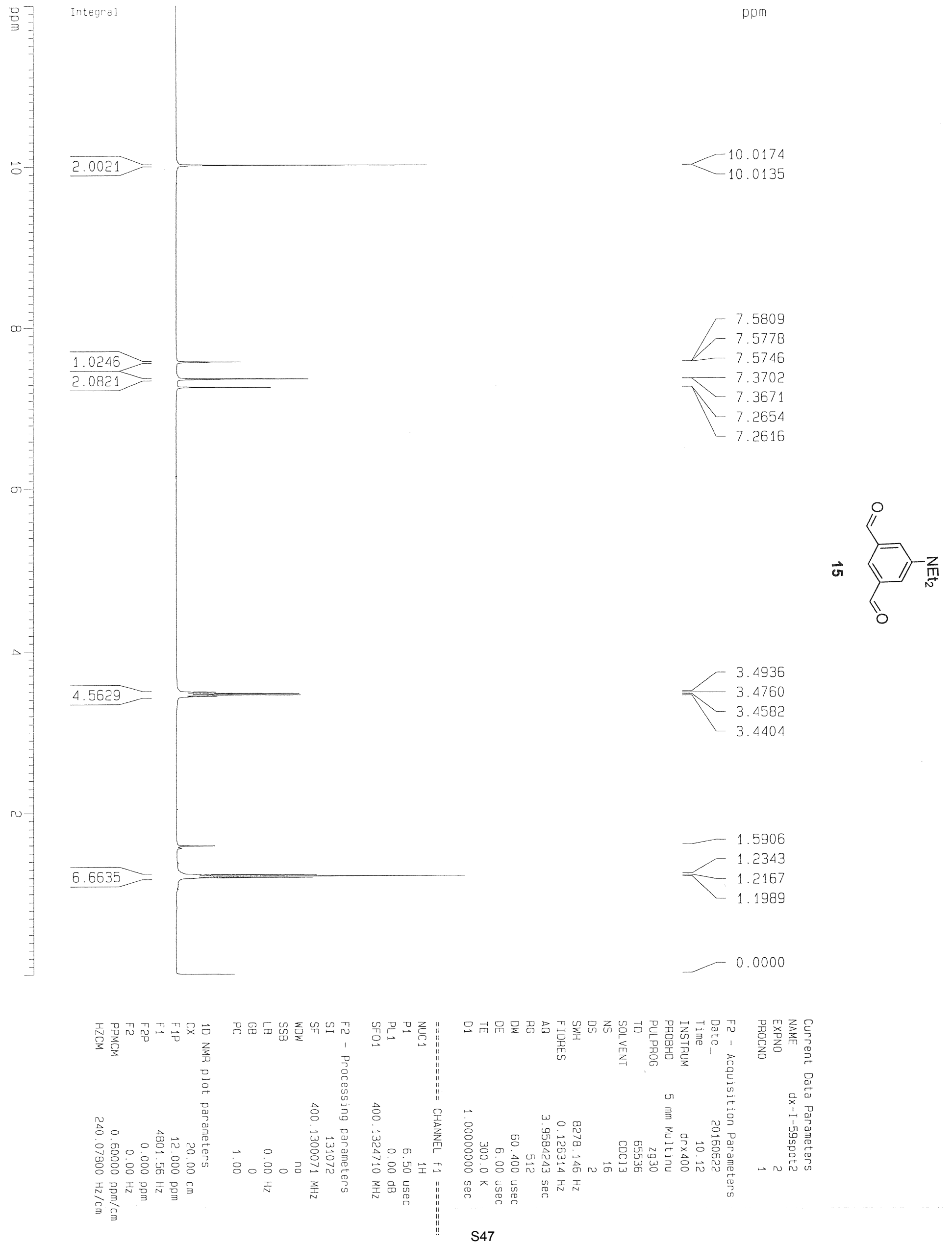

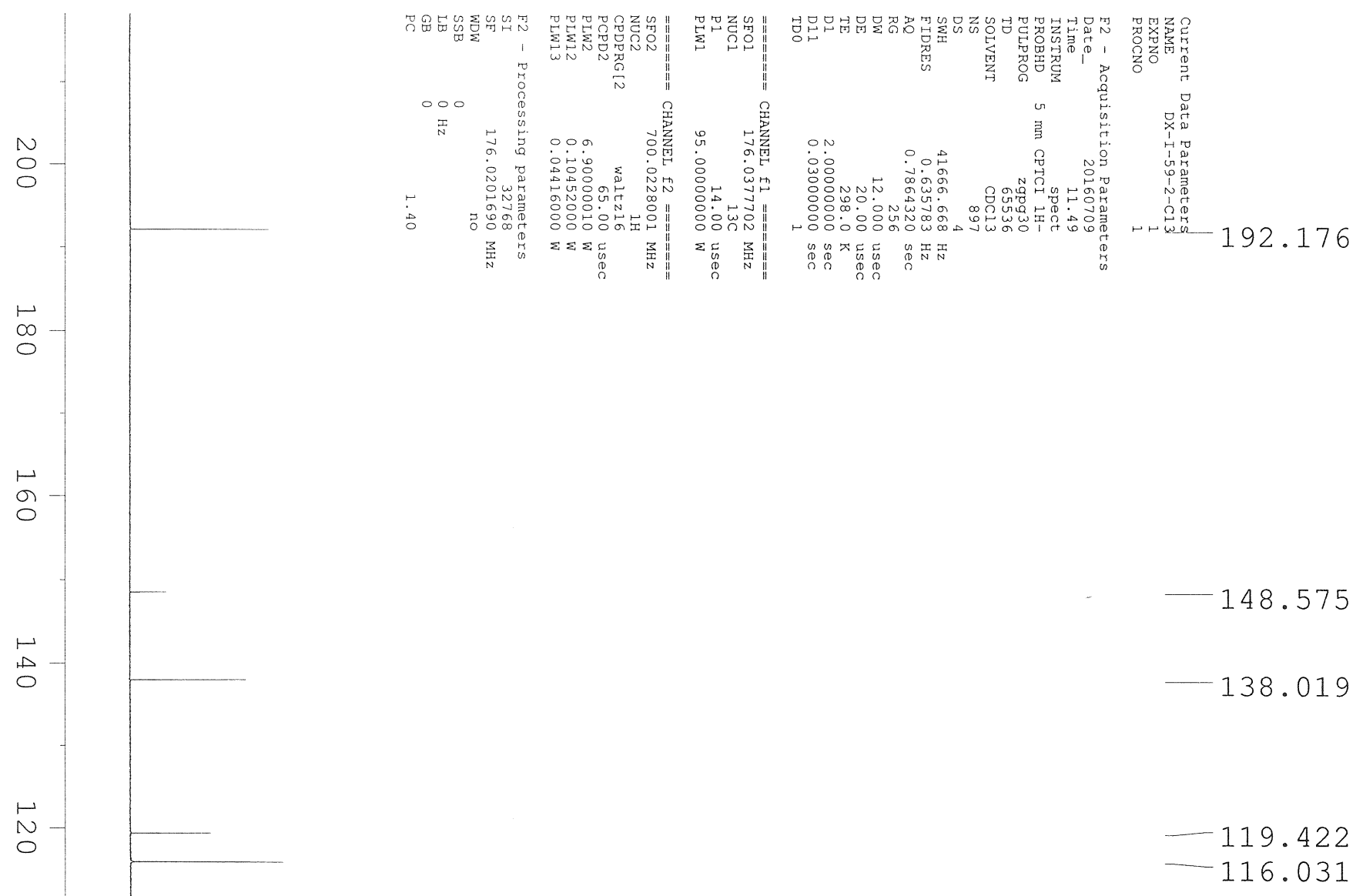

॰

$\infty$

77.220

77.039

76.857

o

ㅇ-

8

$\overrightarrow{0}$

$-44.561$

$-12.322$ 


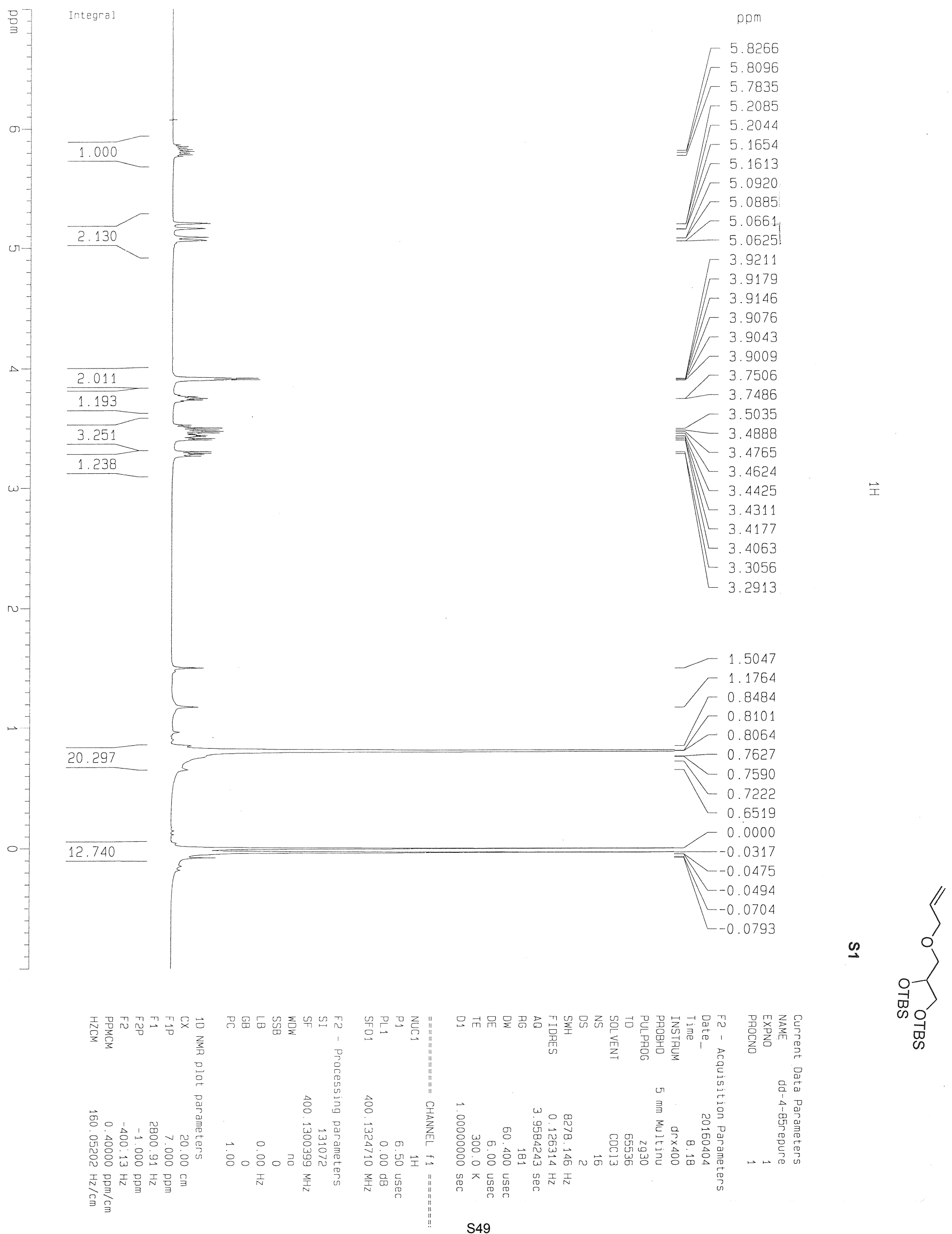



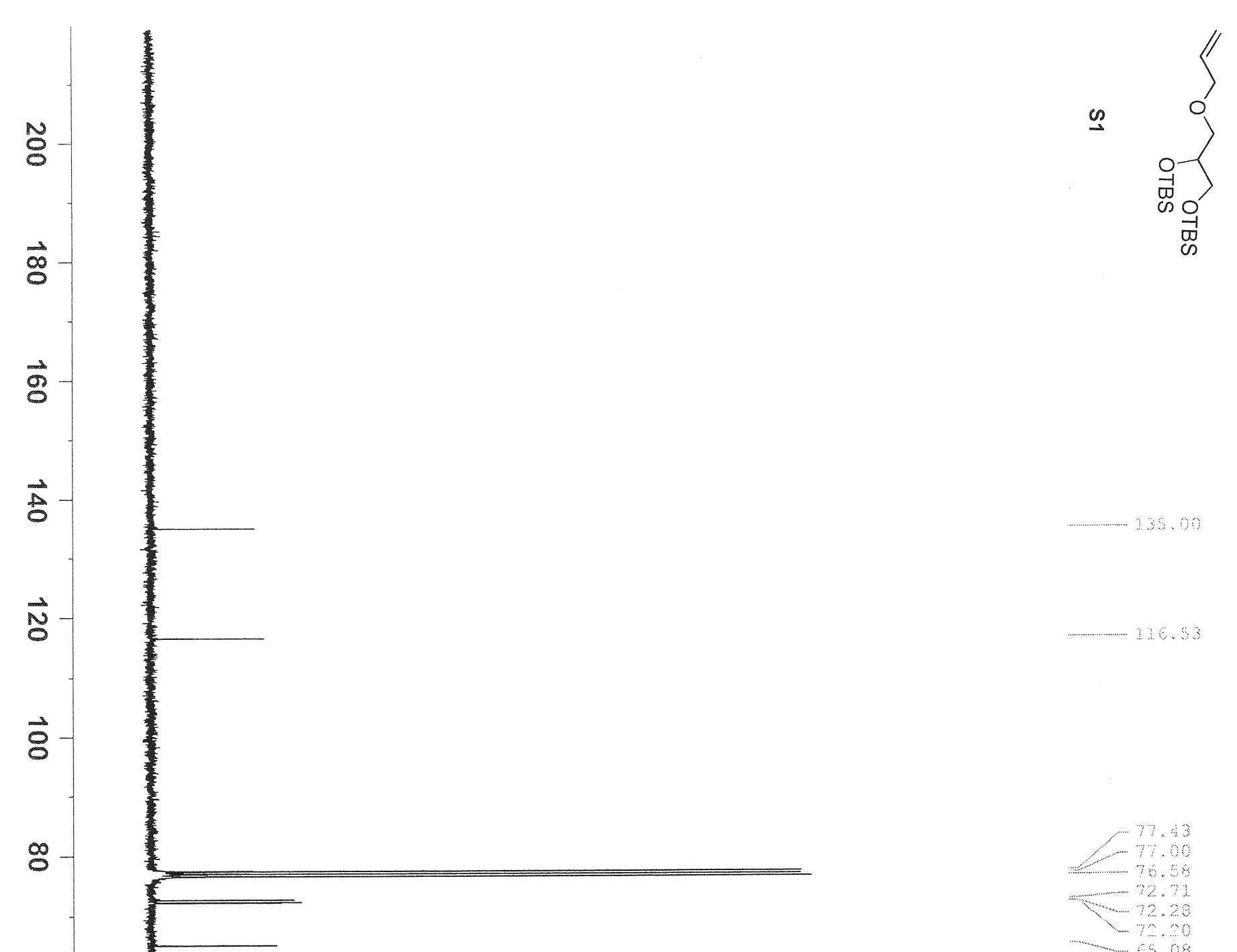